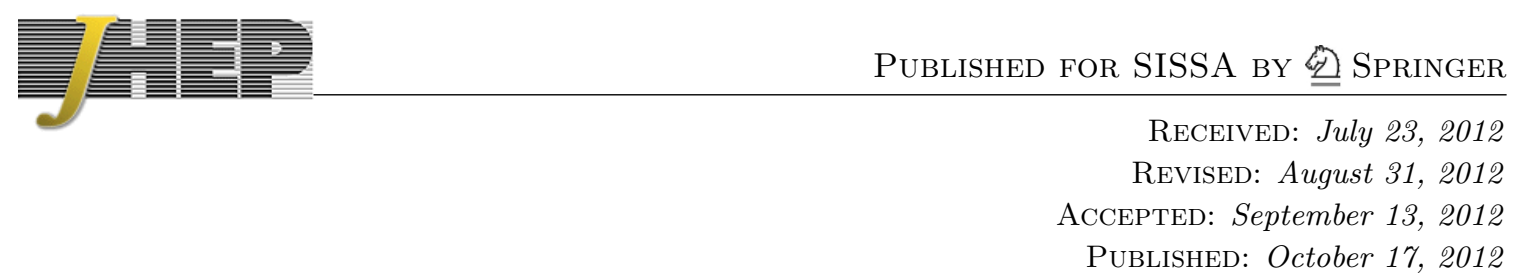

\title{
Higgs boson masses in the complex NMSSM at one-loop level
}

\author{
T. Graf, ${ }^{a}$ R. Gröber, ${ }^{a}$ M. Mühlleitner, ${ }^{a}$ H. Rzehak ${ }^{b, 1}$ and K. Walz ${ }^{a}$ \\ ${ }^{a}$ Institut für Theoretische Physik, Karlsruhe Institute of Technology, \\ 76128 Karlsruhe, Germany \\ ${ }^{b}$ CERN, PH-TH, 1211 Geneva 23, Switzerland \\ E-mail: thorben_graf@gmx.de, ramona@particle.uni-karlsruhe.de , \\ maggie@particle.uni-karlsruhe.de, heidi.rzehak@cern.ch , \\ kwalz@particle.uni-karlsruhe.de
}

AbStract: The Next-to-Minimal Supersymmetric Extension of the Standard Model (NMSSM) with a Higgs sector containing five neutral and two charged Higgs bosons allows for a rich phenomenology. In addition, the plethora of parameters provides many sources of $\mathrm{CP}$ violation. In contrast to the Minimal Supersymmetric Extension, CP violation in the Higgs sector is already possible at tree-level. For a reliable understanding and interpretation of the experimental results of the Higgs boson search, and for a proper distinction of Higgs sectors provided by the Standard Model or possible extensions, the Higgs boson masses have to be known as precisely as possible including higher-order corrections. In this paper we calculate the one-loop corrections to the neutral Higgs boson masses in the complex NMSSM in a Feynman diagrammatic approach adopting a mixed renormalization scheme based on on-shell and $\overline{\mathrm{DR}}$ conditions. We study various scenarios where we allow for tree-level CP-violating phases in the Higgs sector and where we also study radiatively induced $\mathrm{CP}$ violation due to a non-vanishing phase of the trilinear coupling $A_{t}$ in the stop sector. The effects on the Higgs boson phenomenology are found to be significant. We furthermore estimate the theoretical error due to unknown higher-order corrections by both varying the renormalization scheme of the top and bottom quark masses and by adopting different renormalization scales. The residual theoretical error can be estimated to about $10 \%$.

KEYwords: Higgs Physics, Extended Supersymmetry

\footnotetext{
${ }^{1}$ On leave from: Albert-Ludwigs-Universität Freiburg, Physikalisches Institut, Freiburg, Germany.
} 


\section{Contents}

1 Introduction 1

2 Complex parameters in the NMSSM 4

$\begin{array}{lll}3 & \text { The Higgs boson sector in the complex NMSSM } & 7\end{array}$

$\begin{array}{lll}3.1 & \text { The Higgs boson sector at tree-level } & 7\end{array}$

$\begin{array}{lll}3.2 & \text { Set of input parameters for the Higgs boson sector } & 10\end{array}$

$\begin{array}{ll}3.3 \text { The Higgs boson sector at one-loop level } & 10\end{array}$

$\begin{array}{ll}\text { 3.3.1 Higgs boson field renormalization } & 11\end{array}$

$\begin{array}{ll}\text { 3.3.2 Parameter renormalization } & 12\end{array}$

3.4 Loop corrected Higgs boson masses and mixing matrix elements 18

4 Numerical analysis $\quad 19$

4.1 Scenario with a SM-like $H_{3} \quad 21$

4.1.1 CP violation at tree-level 22

4.1.2 No tree-level CP violation 24

4.1.3 Radiatively induced CP violation through the stop sector 25

4.2 Scenario with SM-like $H_{1}$ or $H_{2} \quad 27$

$5 \quad$ Summary and conclusions $\quad 30$

$\begin{array}{ll}\text { A Relations between original and physical parameters } & 31\end{array}$

$\begin{array}{ll}\text { B Higgs boson mass matrix } & 31\end{array}$

$\begin{array}{ll}\text { C Chargino and neutralino self-energies } & 33\end{array}$

\section{Introduction}

The search for the Higgs boson and ultimately the understanding of the mechanism behind the creation of particle masses represents one of the major goals of the Large Hadron Collider (LHC). Recently, the experimental collaborations ATLAS and CMS have updated their results on the search for the Higgs boson. Both experiments observe an excess of events in the low Higgs mass range, compatible with a Standard Model (SM) Higgs boson mass hypothesis close to $124 \mathrm{GeV}$ at $3.1 \sigma$ local significance as reported by CMS [1-9] and close to $126 \mathrm{GeV}$ at $3.5 \sigma$ local significance in the ATLAS experiment [10-17]. This is still too far away from the $5 \sigma$ required to claim discovery and necessitates the accumulation of further data in the ongoing experiment. The slight excess of events in the $\gamma \gamma$ final state signature as compared to the Standard Model expectation may hint to the existence of 
new physics. One of the most popular extensions of the SM are supersymmetric models (SUSY) [18-32]. While the Higgs sector of the Minimal Supersymmetric Extension (MSSM) [33-38] consists of two complex Higgs doublets, which lead to five physical Higgs states after electroweak symmetry breaking (EWSB), the Next-to-Minimal Supersymmetric Model (NMSSM) [39-54] extends the Higgs sector by an additional singlet superfield $\hat{S}$. This entails 7 Higgs bosons after EWSB, which in the limit of the real NMSSM can be divided into three neutral purely CP-even, two neutral purely CP-odd and two charged Higgs bosons, and in total leads to five neutralinos. Although more complicated than the MSSM, the NMSSM has several attractive features. Thus it allows for the dynamical solution of the $\mu$ problem [55] through the scalar component of the singlet field acquiring a non-vanishing vacuum expectation value. Furthermore, the tree-level mass value of the lightest Higgs boson is increased by new contributions to the quartic coupling so that the radiative corrections necessary to shift the Higgs mass to $\sim 125 \mathrm{GeV}$ are less important than in the MSSM allowing for lighter stop masses ${ }^{1}$ and less finetuning [56-61]. The enlarged Higgs and neutralino sectors, finally, lead to a richer phenomenology both in collider and dark matter (DM) experiments. The latter is due to the possibility of a singlino-like lightest neutralino, the former due to heavier Higgs bosons decaying into lighter ones at sizeable rates or due to possibly enhanced or suppressed branching ratios in LHC standard search channels such as $\gamma \gamma$ or vector boson final states [61-64], to cite only a few of the possible modifications compared to SM or MSSM phenomenology.

The enlarged parameter set in supersymmetric theories allows for further sources of $\mathrm{CP}$ violation as compared to the SM, where the only source of $\mathrm{CP}$ violation occurs in the CKM matrix. Hence, the soft SUSY breaking couplings and gaugino masses as well as the Higgsino mixing parameter $\mu$ can be complex. While in the MSSM CP violation in the Higgs sector is not possible at tree-level due to the minimality conditions of the Higgs potential, it can be radiatively induced through non-vanishing CP phases [65-79]. Consequently, the CP-even and CP-odd Higgs bosons mix so that the physical states have no definite $\mathrm{CP}$ quantum number any more leading to substantial modifications in Higgs boson phenomenology [80-85]. The Higgs couplings to the SM gauge bosons and fermions, their SUSY partners and the Higgs self-couplings can be considerably modified compared to the CP-conserving case inducing significant changes in the Higgs boson production rates and decay modes. This could allow for Higgs bosons with masses below present exclusion bounds from LEP and possibly Tevatron and LHC as they might have escaped detection due to suppressed couplings involved in the various standard Higgs search channels, which would then necessitate new search strategies [86-100].

In the NMSSM CP violation in the Higgs sector is possible at tree-level. Though spontaneous tree-level CP violation in the $Z_{3}$-invariant NMSSM is impossible due to vacuum stability [101], explicit CP violation can be realized already at tree-level in contrast to the MSSM. In principle, there can be six complex phases parametrizing the $\mathrm{CP}$ violation in the Higgs sector, two relative phases between the vacuum expectation values (VEVs) of the Higgs doublet and singlet fields and four phases for the complex parameters $\lambda, \kappa, A_{\lambda}, A_{\kappa}$.

\footnotetext{
${ }^{1}$ The bulk of the radiative corrections stems from the (s)top loops.
} 
At tree-level these phases appear only in certain combinations, however, and exploiting tadpole conditions we are left with only one independent phase combination. Explicit CP violation in the Higgs sector leads to potentially large corrections to the electric dipole moments (EDMs). The non-observation of EDMs for thallium, neutron and mercury [102-104] severely constrains the CP-violating phases. However, as the phase combinations occurring in the EDMs can be different from the ones inducing Higgs mixing, the phases can be chosen such that the contributions to the EDMs are small while the phases important for the Higgs sector can still be sizeable [105-109]. This provides additional CP violation necessary for electroweak baryogenesis [110]. The explicit tree-level CP violation induces scalar-pseudoscalar mixings between the doublet fields $H_{u, d}$ and the singlet field $S$, but not between the scalar and pseudoscalar components of the Higgs doublets $H_{u, d}$. The latter is realized in scenarios where explicit $\mathrm{CP}$ violation in the Higgs sector is induced through radiative corrections. Radiative $\mathrm{CP}$ violation furthermore allows for a moderate amount of CP violation which is still in agreement with the bounds from the EDMs [111]. In this respect, the CP phases which play a role are $\varphi_{A_{t}}, \varphi_{A_{b}}$ from the trilinear couplings $A_{t}, A_{b}$. They are involved in the dominant corrections from the third generation squark loops. Phases from third generation Yukawa couplings on the other hand can be reabsorbed by redefinitions of the quark fields when neglecting generation mixing. Further sources for radiative CP violation stem from the gaugino sector where the soft SUSY breaking mass parameters $M_{1}, M_{2}$ and $M_{3}$ are in general complex. One of the two parameters $M_{1}$ and $M_{2}$ can be chosen real by applying an $R$-symmetry transformation. The gluino mass parameter $M_{3}$ and hence its phase enters only at the two-loop level.

Radiatively induced CP-violating effects from the third generation squark sector have been considered in the effective potential approach at one-loop level in refs. [112, 113]. One-loop contributions from the charged particle loops have been taken into account by refs. [114, 115], also in the effective potential approach. In ref. [116] the third generation (s)quark and gauge contributions are included in the one-loop effective potential. The full one-loop and logarithmically enhanced two-loop effects in the renormalization-group improved approach have been included in [117]. In order to properly interpret the results from the experiments and distinguish the various Higgs sectors from each other, a precise knowledge of the Higgs boson masses and couplings at the highest possible accuracy, including higher-order corrections, is indispensable. In this paper we consider the full oneloop corrections to the Higgs boson masses in the CP-violating NMSSM in the Feynman diagrammatic approach. ${ }^{2}$ We allow for explicit CP violation at tree-level by including nonvanishing $\mathrm{CP}$ phases for the Higgs doublets and singlet, as well as for $\lambda, \kappa, A_{\lambda}$ and $A_{\kappa}$, which effectively reduce to one physical CP phase combination at tree-level when the tadpole conditions are exploited. We furthermore allow for radiatively induced CP violation stemming from the stop ${ }^{3}$ by choosing a non-vanishing CP phase for $A_{t}$. The Higgs sector as well as the neutralino and chargino sector will be used to determine the counterterms. The renormalization is performed in a mixed scheme which combines $\overline{\mathrm{DR}}$ conditions for

\footnotetext{
${ }^{2}$ Higher-order corrections to the Higgs boson masses in the real NMSSM can be found in refs. [118-127].

${ }^{3}$ For the low values of $\tan \beta$ applied in our numerical analysis, the $\mathrm{CP}$-violating effects from the sbottom sector are marginal.
} 
the parameters not directly related to physical observables with on-shell (OS) conditions for the physical input values. For the choice of our parameter sets the recent constraints from the Higgs boson searches at LEP [128, 129], Tevatron [130] and LHC [1-17] are taken into account. The inclusion of $\mathrm{CP}$ violation affects the Higgs phenomenology and hence the validity of possible scenarios. Our results will therefore help to clarify the question what kind of Higgs sector may be realized in nature for a SM-like Higgs boson with mass around $125 \mathrm{GeV}$, should the tantalizing hints of the LHC experiments be confirmed by the discovery of the Higgs boson at the $5 \sigma$ level once a sufficient amount of data is accumulated.

The organization of the paper is as follows. In section 2 the parameters of the complex NMSSM will be introduced. Section 3 presents the details of our calculation. After introduction of the complex tree-level Higgs sector in section 3.1 the set of input parameters is given in section 3.2. The renormalization conditions and determination of the Higgs masses as well as the mixing angles are discussed in sections 3.3 and 3.4, respectively. In section 4 we present our numerical analysis, discuss the influence of different renormalization schemes as well as the consequences of one-loop corrections in the complex NMSSM for the Higgs boson phenomenology and results at the LHC. We terminate with the conclusions in section 5 .

\section{Complex parameters in the NMSSM}

The Lagrangian of the complex NMSSM can be divided into an MSSM part which is adopted from the MSSM Lagrangian and an additional NMSSM part. The latter contains (apart from the phases of the Higgs doublets and singlets) four additional complex parameters. Two of them are the coupling $\kappa$ of the self-interaction of the new singlet superfield $\hat{S}$ and the coupling $\lambda$ for the interaction of $\hat{S}$ with the Higgs doublet superfields $\hat{H}_{u}$ and $\hat{H}_{d}\left(\hat{H}_{u}\right.$ and $\hat{H}_{d}$ couple to the up- and down-type quark superfields, respectively). They are introduced via the extension of the MSSM superpotential $W_{\text {MSSM }}$,

$$
W_{\mathrm{NMSSM}}=W_{\mathrm{MSSM}}-\epsilon_{a b} \lambda \hat{S} \hat{H}_{d}^{a} \hat{H}_{u}^{b}+\frac{1}{3} \kappa \hat{S}^{3},
$$

with $\epsilon_{12}=\epsilon^{12}=1$. The MSSM part of the superpotential is given by

$$
W_{\mathrm{MSSM}}=-\epsilon_{a b}\left(y_{u} \hat{H}_{u}^{a} \hat{Q}^{b} \hat{U}^{c}-y_{d} \hat{H}_{d}^{a} \hat{Q}^{b} \hat{D}^{c}-y_{e} \hat{H}_{d}^{a} \hat{L}^{b} \hat{E}^{c}\right),
$$

where $\hat{Q}$ and $\hat{L}$ are the left-handed quark and lepton superfield doublets and $\hat{U}, \hat{D}$ and $\hat{E}$ are the right-handed up-type, down-type and electron-type superfield singlets, respectively. The superscript $c$ denotes charge conjugation. Colour and generation indices have been omitted. The quark and lepton Yukawa couplings are given by $y_{d}, y_{u}$ and $y_{e}$. They are in general complex. However, when neglecting generation mixing, as we assume in this paper, the phases of the Yukawa couplings can be reabsorbed by redefining the quark fields i.e. these phases can be chosen arbitrarily without changing the physical meaning [131].

The soft SUSY breaking Lagrangian in the NMSSM is also extended with respect to the MSSM,

$$
\mathcal{L}_{\mathrm{NMSSM}}^{\mathrm{soft}}=\mathcal{L}_{\mathrm{MSSM}}^{\mathrm{soft}}-m_{S}^{2}|S|^{2}+\left(\epsilon_{a b} A_{\lambda} \lambda S H_{d}^{a} H_{u}^{b}-\frac{1}{3} A_{\kappa} \kappa S^{3}+\text { h.c. }\right),
$$


containing two further complex parameters specific to the NMSSM, the soft SUSY breaking trilinear couplings $A_{\lambda}$ and $A_{\kappa}$. The MSSM part is given by ${ }^{4}$

$$
\begin{aligned}
\mathcal{L}_{\mathrm{MSSM}}^{\mathrm{soft}}= & -m_{H_{d}}^{2}\left|H_{d}\right|^{2}-m_{H_{u}}^{2}\left|H_{u}\right|^{2}-m_{Q}^{2}|\tilde{Q}|^{2}-m_{U}^{2}\left|\tilde{u}_{R}\right|^{2}-m_{D}^{2}\left|\tilde{d}_{R}\right|^{2}-m_{L}^{2}|\tilde{L}|^{2}-m_{E}^{2}\left|\tilde{e}_{R}\right|^{2} \\
& +\epsilon_{a b}\left(y_{u} A_{u} H_{u}^{a} \tilde{Q}^{b} \tilde{u}_{R}^{*}-y_{d} A_{d} H_{d}^{a} \tilde{Q}^{b} \tilde{d}_{R}^{*}-y_{e} A_{e} H_{d}^{a} \tilde{Q}^{b} \tilde{e}_{R}^{*}+h . c .\right) \\
& -\frac{1}{2}\left(M_{1} \tilde{B} \tilde{B}+M_{2} \tilde{W}_{i} \tilde{W}_{i}+M_{3} \tilde{G} \tilde{G}+h . c\right) .
\end{aligned}
$$

The soft SUSY breaking trilinear couplings $A_{u}, A_{d}, A_{e}$ of the up-type, down-type and charged lepton-type sfermions, ${ }^{5}$ respectively, which are already present in the MSSM, are in general complex. However, the soft SUSY breaking mass parameters of the scalar fields, $m_{X}^{2}\left(X=S, H_{d}, H_{u}, Q, U, D, L, E\right)$, are real. The SM-type and SUSY fields forming a superfield (denoted with a hat) are represented by a letter without and with a tilde, respectively: $\tilde{Q}, \tilde{L}$ and $\tilde{u}_{R}, \tilde{d}_{R}, \tilde{e}_{R}$ are the superpartner fields corresponding to the leftand right-handed quark and lepton fields. In general, also the soft SUSY breaking mass parameters of the gauginos, $M_{1}, M_{2}$ and $M_{3}$, are complex where the gaugino fields are denoted by $\tilde{B}, \tilde{W}_{i}(i=1,2,3)$ and $\tilde{G}$ for the bino, the winos and the gluinos corresponding to the weak hypercharge $\mathrm{U}(1)$, the weak isospin $\mathrm{SU}(2)$ and the colour $\mathrm{SU}(3)$ symmetry. The $R$-symmetry can then be exploited to choose either $M_{1}$ or $M_{2}$ to be real. The kinetic and gauge interaction part of the NMSSM Lagrangian finally do not contain any complex parameters.

Expressing the Higgs boson fields as an expansion about the vacuum expectation values, two further phases appear,

$$
H_{d}=\left(\begin{array}{c}
\frac{1}{\sqrt{2}}\left(v_{d}+h_{d}+i a_{d}\right) \\
h_{d}^{-}
\end{array}\right), \quad H_{u}=e^{i \varphi_{u}}\left(\begin{array}{c}
h_{u}^{+} \\
\frac{1}{\sqrt{2}}\left(v_{u}+h_{u}+i a_{u}\right)
\end{array}\right), \quad S=\frac{e^{i \varphi_{s}}}{\sqrt{2}}\left(v_{s}+h_{s}+i a_{s}\right) .
$$

The phases $\varphi_{u}$ and $\varphi_{s}$ describe the phase differences between the three vacuum expectation values $\left\langle H_{d}^{0}\right\rangle,\left\langle H_{u}^{0}\right\rangle$ and $\langle S\rangle$. In case of vanishing phases, $\varphi_{u}=\varphi_{s}=0$, the fields $h_{i}$ and $a_{i}$ with $i=d, u, s$ correspond to the CP-even and CP-odd part of the neutral entries of $H_{u}$, $H_{d}$ and $S$. The charged components are denoted by $h_{i}^{ \pm}(i=d, u)$.

Exploiting that the phases of the Yukawa couplings can be chosen arbitrarily, the phase of the up-type coupling is set to $\varphi_{y_{u}}=-\varphi_{u}$ while the down-type and the charged leptontype ones are assumed to be real. This choice ensures that the quark and lepton mass terms yield real masses without any further phase transformation of the corresponding fields.

In the following renormalization procedure we will make use of the chargino and neutralino sectors, therefore they are introduced briefly here. The fermionic superpartners of the neutral Higgs bosons and colourless gauge bosons are $\tilde{H}_{d}^{0}$ and $\tilde{H}_{u}^{0}$ for the neutral

\footnotetext{
${ }^{4}$ Here the indices of the soft SUSY breaking masses, $Q(L)$ stand for the left-handed doublet of the three quark (lepton) generations and $U, D, E$ are the indices for the right-handed up-type and down-type fermions and charged leptons, respectively. In the trilinear coupling parameters the indices $u, d, e$ represent the up-type and down-type fermions and charged leptons.

${ }^{5}$ We neglect generation mixings so that we have nine complex numbers $A_{u}, A_{d}, A_{e}$ instead of three complex $3 \times 3$ matrices.
} 
components of the Higgs doublets, $\tilde{S}$ for the Higgs singlet, the bino $\tilde{B}$ and the neutral component $\tilde{W}_{3}$ of the winos. After electroweak symmetry breaking these fields mix, and in the Weyl spinor basis $\psi^{0}=\left(\tilde{B}, \tilde{W}_{3}, \tilde{H}_{d}^{0}, \tilde{H}_{u}^{0}, \tilde{S}\right)^{T}$ the neutralino mass matrix $M_{N}$ can be written as

$$
\begin{aligned}
& M_{N}= \\
& \left(\begin{array}{ccccc}
M_{1} & 0 & -c_{\beta} M_{Z} s_{\theta_{W}} & M_{Z} s_{\beta} s_{\theta_{W}} e^{-i \varphi_{u}} & 0 \\
0 & M_{2} & c_{\beta} M_{W} & -M_{W} s_{\beta} e^{-i \varphi_{u}} & 0 \\
-c_{\beta} M_{Z} s_{\theta_{W}} & c_{\beta} M_{W} & 0 & -\lambda \frac{v_{s}}{\sqrt{2}} e^{i \varphi_{s}} & -\frac{\sqrt{2} M_{W} s_{\beta} s_{\theta_{W}} \lambda e^{i \varphi_{u}}}{e} \\
M_{Z} s_{\beta} s_{\theta_{W}} e^{-i \varphi_{u}} & -M_{W} s_{\beta} e^{-i \varphi_{u}} & -\lambda \frac{v_{s}}{\sqrt{2}} e^{i \varphi_{s}} & 0 & -\frac{\sqrt{2} M_{W} c_{\beta} s_{\theta_{W}} \lambda}{e} \\
0 & 0 & -\frac{\sqrt{2} M_{W} s_{\beta} s_{\theta_{W}} \lambda e^{i \varphi_{u}}}{e} & -\frac{\sqrt{2} M_{W} c_{\beta} s_{\theta_{W}} \lambda}{e} & \sqrt{2} \kappa v_{s} e^{i \varphi_{s}}
\end{array}\right)
\end{aligned}
$$

where $M_{W}$ and $M_{Z}$ are the $W$ and $Z$ boson masses, respectively. The angle $\beta$ is defined via the ratio of the vacuum expectation values of the two Higgs doublets, $\tan \beta=v_{u} / v_{d}$, $\theta_{W}$ denotes the electroweak mixing angle and $e$ is the electric charge. From here on the short hand notation $c_{x}=\cos x, s_{x}=\sin x$ and $t_{x}=\tan x$ is used.

The neutralino mass matrix $M_{N}$ is complex ${ }^{6}$ but symmetric and can be diagonalized with the help of the $5 \times 5$ matrix $\mathcal{N}$, yielding $\operatorname{diag}\left(m_{\tilde{\chi}_{1}^{0}}, m_{\tilde{\chi}_{2}^{0}}, m_{\tilde{\chi}_{3}^{0}}, m_{\tilde{\chi}_{4}^{0}}, m_{\tilde{\chi}_{5}^{0}}\right)=\mathcal{N}^{*} M_{N} \mathcal{N}^{\dagger}$, where the absolute mass values are ordered as $\left|m_{\tilde{\chi}_{1}^{0}}\right| \leq \ldots \leq\left|m_{\tilde{\chi}_{5}^{0}}\right|$. The neutralino mass eigenstates $\tilde{\chi}_{i}^{0}$, expressed as a Majorana spinor, can then be obtained by

$$
\tilde{\chi}_{i}^{0}=\left(\chi_{i}^{0}, \overline{\chi_{i}^{0}}\right)^{T} \quad \text { with } \quad \chi_{i}^{0}=\mathcal{N}_{i j} \psi_{j}^{0}, \quad i, j=1, \ldots, 5 .
$$

The fermionic superpartners of the charged Higgs and gauge bosons are given in terms of the Weyl spinors $\tilde{H}_{d}^{ \pm}, \tilde{H}_{u}^{ \pm}, \tilde{W}_{1}$ and $\tilde{W}_{2}$ where the latter two can be reexpressed as $\tilde{W}^{ \pm}=\left(\tilde{W}_{1} \mp i \tilde{W}_{2}\right) / \sqrt{2}$. Arranging these Weyl spinors as

$$
\psi_{R}^{-}=\left(\begin{array}{c}
\tilde{W}^{-} \\
\tilde{H}_{d}^{-}
\end{array}\right), \quad \psi_{L}^{+}=\left(\begin{array}{c}
\tilde{W}^{+} \\
\tilde{H}_{u}^{+}
\end{array}\right)
$$

leads to mass terms of the form, $\left(\psi_{R}^{-}\right)^{T} M_{C} \psi_{L}^{+}+$h.c., with the chargino mass matrix

$$
M_{C}=\left(\begin{array}{cc}
M_{2} & \sqrt{2} s_{\beta} M_{W} e^{-i \varphi_{u}} \\
\sqrt{2} c_{\beta} M_{W} & \lambda \frac{v_{s}}{\sqrt{2}} e^{i \varphi_{s}}
\end{array}\right) .
$$

The chargino mass matrix can be diagonalized with the help of two unitary $2 \times 2$ matrices, $U$ and $V$, yielding $\operatorname{diag}\left(m_{\tilde{\chi}_{1}^{ \pm}}, m_{\tilde{\chi}_{2}^{ \pm}}\right)=U^{*} M_{C} V^{\dagger}$ with $m_{\tilde{\chi}_{1}^{ \pm}} \leq m_{\tilde{\chi}_{2}^{ \pm}}$. The left-handed and the right-handed part of the mass eigenstates are

$$
\tilde{\chi}_{L}^{+}=V \psi_{L}^{+}, \quad \tilde{\chi}_{R}^{-}=U \psi_{R}^{-},
$$

respectively, with the mass eigenstates $\tilde{\chi}_{i}^{+}=\left(\tilde{\chi}_{L_{i}}^{+}, \overline{\tilde{\chi}_{R_{i}}^{-}}\right)^{T}, i=1,2$, written as a Dirac spinor.

\footnotetext{
${ }^{6}$ Note, that in general the parameters $\lambda, \kappa, M_{1}$ and $M_{2}$ are complex.
} 


\section{The Higgs boson sector in the complex NMSSM}

\subsection{The Higgs boson sector at tree-level}

To ensure the minimum of the Higgs potential $V_{\text {Higgs }}$ at non-vanishing vacuum expectation values $v_{u}, v_{d}, v_{s}$ the terms linear in the Higgs boson fields have to vanish according to

$$
\left.t_{\phi} \equiv \frac{\partial V_{\text {Higgs }}}{\partial \phi}\right|_{\text {Min. }} \stackrel{!}{=} 0 \quad \text { for } \quad \phi=h_{d}, h_{u}, h_{s}, a_{d}, a_{u}, a_{s}
$$

At tree-level, these tadpole parameters $t_{\phi}$ are given by ${ }^{7}$

$$
\begin{aligned}
& t_{h_{d}}=\left[m_{H_{d}}^{2}+\frac{M_{Z}^{2} c_{2 \beta}}{2}-v_{s} t_{\beta}|\lambda|\left(\frac{\left|A_{\lambda}\right|}{\sqrt{2}} c_{\varphi_{x}}+|\kappa| \frac{v_{s}}{2} c_{\varphi_{y}}\right)+|\lambda|^{2}\left(\frac{2 s_{\beta}^{2} M_{W}^{2} s_{\theta_{W}}^{2}}{e^{2}}+\frac{v_{s}^{2}}{2}\right)\right] \frac{2 c_{\beta} M_{W} s_{\theta_{W}}}{e}, \\
& t_{h_{u}}=\left[m_{H_{u}}^{2}-\frac{M_{Z}^{2} c_{2 \beta}}{2}-\frac{|\lambda| v_{s}}{t_{\beta}}\left(\frac{\left|A_{\lambda}\right|}{\sqrt{2}} c_{\varphi_{x}}+|\kappa| \frac{v_{s}}{2} c_{\varphi_{y}}\right)+|\lambda|^{2}\left(\frac{2 c_{\beta}^{2} M_{W}^{2} s_{\theta_{W}}^{2}}{e^{2}}+\frac{v_{s}^{2}}{2}\right)\right] \frac{2 s_{\beta} M_{W} s_{\theta_{W}}}{e},
\end{aligned}
$$

$t_{h_{s}}=m_{S}^{2} v_{s}-\left[s_{2 \beta}|\lambda|\left(\frac{\left|A_{\lambda}\right|}{\sqrt{2}} c_{\varphi_{x}}+|\kappa| v_{s} c_{\varphi_{y}}\right)-|\lambda|^{2} v_{s}\right] \frac{2 M_{W}^{2} s_{\theta_{W}}^{2}}{e^{2}}+|\kappa|^{2} v_{s}^{3}+\frac{1}{\sqrt{2}}\left|A_{\kappa}\right||\kappa| v_{s}^{2} c_{\varphi_{z}}$

$t_{a_{d}}=\frac{M_{W} s_{\theta_{W}} s_{\beta}}{e}|\lambda| v_{s}\left(\sqrt{2}\left|A_{\lambda}\right| s_{\varphi_{x}}-|\kappa| v_{s} s_{\varphi_{y}}\right)$,

$t_{a_{u}}=\frac{1}{t_{\beta}} t_{a_{d}}$,

$t_{a_{s}}=\frac{2 M_{W}^{2} s_{\theta_{W}}^{2} s_{2 \beta}}{e^{2}}|\lambda|\left(\frac{1}{\sqrt{2}}\left|A_{\lambda}\right| s_{\varphi_{x}}+|\kappa| v_{s} s_{\varphi_{y}}\right)-\frac{1}{\sqrt{2}}\left|A_{\kappa}\right||\kappa| v_{s}^{2} s_{\varphi_{z}}$,

where we have introduced a short hand notation for the following phase combinations

$$
\begin{aligned}
& \varphi_{x}=\varphi_{A_{\lambda}}+\varphi_{\lambda}+\varphi_{s}+\varphi_{u}, \\
& \varphi_{y}=\varphi_{\kappa}-\varphi_{\lambda}+2 \varphi_{s}-\varphi_{u}, \\
& \varphi_{z}=\varphi_{A_{\kappa}}+\varphi_{\kappa}+3 \varphi_{s} .
\end{aligned}
$$

In the expressions for the tadpole parameters some of the original parameters have already been replaced in favour of the parameters on which we will impose our renormalization conditions, as described in detail in sections 3.2 and 3.3. Thus the vacuum expectation values $v_{u}, v_{d}$ and the $\mathrm{U}(1)$ and $\mathrm{SU}(2)$ gauge couplings $g^{\prime}$ and $g$ have been replaced by $\tan \beta=v_{u} / v_{d}$, the gauge boson masses $M_{W}$ and $M_{Z}$ and the electric charge $e$ (according to eqs. (A.7) and (A.8) in appendix A). This replacement has also been applied in the expressions of the mass matrices given below.

It should be noted that the eqs. (3.5) and (3.7) can have zero, one or two solutions for $\varphi_{x}, \varphi_{z} \in[-\pi, \pi)$ depending on the values of the parameters. If no solution is found

\footnotetext{
${ }^{7}$ The complex parameters are expressed in terms of their absolute value and a complex phase, i.e. for example $\lambda \equiv|\lambda| e^{i \varphi_{\lambda}}$.
} 
there is no minimum of the Higgs potential at the corresponding set of values $v_{d}, v_{u}, v_{s}$ and thus this parameter point is discarded. The single solutions yield one of the two values $\varphi_{x}, \varphi_{z}= \pm \pi / 2$. Assuming there exist two solutions of eq. (3.5) then if $\varphi_{x}^{S}$ with $\varphi_{x}^{S}>0$ solves this equation then also $\pi-\varphi_{x}^{S}$ is a solution and similarly if $\varphi_{x}^{S}$ with $\varphi_{x}^{S}<0$ is a solution then $-\left(\pi-\varphi_{x}^{S}\right)$ is the second solution, analogously for $\varphi_{z}^{S}$.

The terms of the Higgs potential which are bilinear in the neutral Higgs boson fields contribute to the corresponding $6 \times 6$ Higgs boson mass matrix $M_{\phi \phi}$ in the basis of $\phi=$ $\left(h_{d}, h_{u}, h_{s}, a_{d}, a_{u}, a_{s}\right)^{T}$ which can be expressed in terms of three $3 \times 3$ matrices $M_{h h}, M_{a a}$ and $M_{h a}$

$$
M_{\phi \phi}=\left(\begin{array}{cc}
M_{h h} & M_{h a} \\
M_{h a}^{T} & M_{a a}
\end{array}\right)
$$

where $M_{h h}$ and $M_{a a}$ are symmetric matrices.

The entries of $M_{h h}$ describing the mixing of the CP-even components of the Higgs doublet and singlet fields read

$$
\begin{aligned}
& M_{h_{d} h_{d}}=M_{Z}^{2} c_{\beta}^{2}+\frac{1}{2}|\lambda| v_{s} t_{\beta}\left(\sqrt{2}\left|A_{\lambda}\right| c_{\varphi_{x}}+|\kappa| v_{s} c_{\varphi_{y}}\right), \\
& M_{h_{d} h_{u}}=-\frac{1}{2} M_{Z}^{2} s_{2 \beta}-\frac{1}{2}|\lambda| v_{s}\left(\sqrt{2}\left|A_{\lambda}\right| c_{\varphi_{x}}+|\kappa| v_{s} c_{\varphi_{y}}\right)+2|\lambda|^{2} \frac{M_{W}^{2} s_{\theta_{W}}^{2}}{e^{2}} s_{2 \beta}, \\
& M_{h_{u} h_{u}}=M_{Z}^{2} s_{\beta}^{2}+\frac{1}{2}|\lambda| \frac{v_{s}}{t_{\beta}}\left(\sqrt{2}\left|A_{\lambda}\right| c_{\varphi_{x}}+|\kappa| v_{s} c_{\varphi_{y}}\right), \\
& M_{h_{d} h_{s}}=2|\lambda|^{2} \frac{M_{W} s_{\theta_{W}}}{e} c_{\beta} v_{s}-|\lambda| \frac{M_{W} s_{\theta_{W}}}{e} s_{\beta}\left(\sqrt{2}\left|A_{\lambda}\right| c_{\varphi_{x}}+2|\kappa| v_{s} c_{\varphi_{y}}\right), \\
& M_{h_{u} h_{s}}=2|\lambda|^{2} \frac{M_{W} s_{\theta_{W}}}{e} s_{\beta} v_{s}-|\lambda| \frac{M_{W} s_{\theta_{W}}}{e} c_{\beta}\left(\sqrt{2}\left|A_{\lambda}\right| c_{\varphi_{x}}+2|\kappa| v_{s} c_{\varphi_{y}}\right), \\
& M_{h_{s} h_{s}}=2|\kappa|^{2} v_{s}^{2}+\frac{v_{s}}{\sqrt{2}}|\kappa|\left|A_{\kappa}\right| c_{\varphi_{z}}+\sqrt{2}\left|A_{\lambda}\right||\lambda| \frac{M_{W}^{2} s_{\theta_{W}}^{2}}{e^{2} v_{s}} s_{2 \beta} c_{\varphi_{x}} .
\end{aligned}
$$

Note, that here the tadpole conditions eqs. (3.2)-(3.4) together with eq. (3.1) have already been applied to eliminate $m_{H_{d}}^{2}, m_{H_{u}}^{2}$ and $m_{S}^{2}$. Exploiting additionally eqs. (3.5) and (3.7) we can eliminate $c_{\varphi_{x}}$ and $c_{\varphi_{z}}$ through

$$
\begin{aligned}
& c_{\varphi_{x}}= \pm \sqrt{1-\frac{|\kappa|^{2} v_{s}^{2}}{2\left|A_{\lambda}\right|^{2}} s_{\varphi_{y}}^{2}}, \\
& c_{\varphi_{z}}= \pm \sqrt{1-18 \frac{M_{W}^{4} s_{\theta_{W}}^{4} s_{2 \beta}^{2}|\lambda|^{2}}{e^{4}\left|A_{\kappa}\right|^{2} v_{s}^{2}} s_{\varphi_{y}}^{2}} .
\end{aligned}
$$

The two signs correspond to the two possible solutions of eqs. (3.5) and (3.7) (as explained before). Choosing either solution will define the sign of the cosine. In our numerical evaluation we will treat the sign as a further input. 
The mixing of the CP-odd components of the Higgs doublet and singlet fields is characterized by the matrix $M_{a a}$ which has the following entries,

$$
\begin{aligned}
M_{a_{d} a_{d}} & =\frac{1}{2}|\lambda|\left(\sqrt{2}\left|A_{\lambda}\right| c_{\varphi_{x}}+|\kappa| v_{s} c_{\varphi_{y}}\right) v_{s} t_{\beta}, \\
M_{a_{d} a_{u}} & =\frac{1}{2}|\lambda|\left(\sqrt{2}\left|A_{\lambda}\right| c_{\varphi_{x}}+|\kappa| v_{s} c_{\varphi_{y}}\right) v_{s}, \\
M_{a_{u} a_{u}} & =\frac{1}{2}|\lambda|\left(\sqrt{2}\left|A_{\lambda}\right| c_{\varphi_{x}}+|\kappa| v_{s} c_{\varphi_{y}}\right) \frac{v_{s}}{t_{\beta}}, \\
M_{a_{d} a_{s}} & =|\lambda| \frac{M_{W} s_{\theta_{W}}}{e} s_{\beta}\left(\sqrt{2}\left|A_{\lambda}\right| c_{\varphi_{x}}-2|\kappa| v_{s} c_{\varphi_{y}}\right), \\
M_{a_{u} a_{s}} & =|\lambda| \frac{M_{W} s_{\theta_{W}}}{e} c_{\beta}\left(\sqrt{2}\left|A_{\lambda}\right| c_{\varphi_{x}}-2|\kappa| v_{s} c_{\varphi_{y}}\right), \\
M_{a_{s} a_{s}} & =|\lambda|\left(\sqrt{2}\left|A_{\lambda}\right| c_{\varphi_{x}}+4|\kappa| v_{s} c_{\varphi_{y}}\right) \frac{M_{W}^{2} s_{\theta_{W}}^{2}}{e^{2} v_{s}} s_{2 \beta}-3\left|A_{\kappa}\right||\kappa| \frac{v_{s}}{\sqrt{2}} c_{\varphi_{z}},
\end{aligned}
$$

where again eq. (3.1) together with the eqs. (3.2)-(3.4) have been applied and eqs. (3.1), (3.5) and (3.7) can be used to replace $c_{\varphi_{x}}$ and $c_{\varphi_{z}}$. The matrix $M_{h a}$ governs the mixing between the CP-even and the CP-odd components of the Higgs doublet and singlet fields,

$$
M_{h a}=\left(\begin{array}{ccc}
0 & 0 & 3 v_{s} s_{\beta} \\
0 & 0 & 3 v_{s} c_{\beta} \\
-v_{s} s_{\beta} & -v_{s} c_{\beta} & -4 s_{2 \beta} \frac{M_{W} s_{\theta_{W}}}{e}
\end{array}\right) \frac{M_{W} s_{\theta_{W}}}{e}|\kappa||\lambda| s_{\varphi_{y}} .
$$

In case of $\varphi_{y}=n_{y} \pi, n_{y} \in \mathbb{Z}$, the entries of $M_{h a}$ vanish and hence, in that case, there is no $\mathrm{CP}$ violation at tree-level in the NMSSM Higgs sector; after transformation to the mass eigenstates we are left with three purely CP-even and two purely CP-odd Higgs bosons.

The transformation into mass eigenstates can be performed in two steps. In our approach, first, the Goldstone boson field is extracted by applying the $6 \times 6$ rotation ma$\operatorname{trix}^{8} \mathcal{R}^{G}$

$$
\Phi_{i}=\mathcal{R}_{i j}^{G} \phi_{j}
$$

where $\Phi=\left(h_{d}, h_{u}, h_{s}, A, a_{s}, G\right)^{T}$. The resulting mass matrix,

$$
M_{\Phi \Phi}=\mathcal{R}^{G} M_{\phi \phi} \mathcal{R}^{G^{T}}
$$

can finally be diagonalized with the help of the matrix $\mathcal{R}$ leading to

$$
\mathcal{R} M_{\Phi \Phi} \mathcal{R}^{T}=\operatorname{diag}\left(\left(M_{H_{1}}^{(0)}\right)^{2}, \ldots,\left(M_{H_{5}}^{(0)}\right)^{2}, 0\right)=: \mathcal{D}_{H}
$$

with the mass values being ordered as $M_{H_{1}}^{(0)} \leq \ldots \leq M_{H_{5}}^{(0)}$ and the superscript (0) denoting the tree-level values of the masses. The corresponding mass eigenstates are obtained as

$$
H_{i}=\mathcal{R}_{i j} \Phi_{j}
$$

\footnotetext{
${ }^{8}$ The explicit form of $\mathcal{R}^{G}$ can be found in appendix B.
} 
The mass matrix $M_{h^{+} h^{-}}$of the charged entries of the Higgs doublet fields,

$$
\left(h_{d}^{+}, h_{u}^{+}\right) M_{h^{+} h^{-}}\left(h_{d}^{-}, h_{u}^{-}\right)^{T},
$$

is explicitly given as

$$
M_{h^{+} h^{-}}=\frac{1}{2}\left(\begin{array}{cc}
t_{\beta} & 1 \\
1 & \frac{1}{t_{\beta}}
\end{array}\right)\left[M_{W}^{2} s_{2 \beta}+|\lambda| v_{s}\left(\sqrt{2}\left|A_{\lambda}\right| c_{\varphi_{x}}+|\kappa| v_{s} c_{\varphi_{y}}\right)-2|\lambda|^{2} \frac{M_{W}^{2} s_{\theta_{W}}^{2}}{e^{2}} s_{2 \beta}\right],
$$

where again the eqs. (3.1)-(3.4) have already been applied. Diagonalizing this mass matrix with the help of a rotation matrix with the angle $\beta_{c}$, where $\beta_{c}=\beta$ at tree-level, yields the mass of the physical charged Higgs boson,

$$
M_{H^{ \pm}}^{2}=M_{W}^{2}+\frac{|\lambda| v_{s}}{s_{2 \beta}}\left(\sqrt{2}\left|A_{\lambda}\right| c_{\varphi_{x}}+|\kappa| v_{s} c_{\varphi_{y}}\right)-2|\lambda|^{2} \frac{M_{W}^{2} s_{\theta_{W}}^{2}}{e^{2}}
$$

and a mass of zero for the charged Goldstone boson.

\subsection{Set of input parameters for the Higgs boson sector}

To summarize, the original parameters entering the Higgs potential and thereby also the Higgs mass matrix are

$$
m_{H_{d}}^{2}, m_{H_{u}}^{2}, m_{S}^{2}, \varphi_{A_{\kappa}}, \varphi_{A_{\lambda}},\left|A_{\lambda}\right|, g, g^{\prime}, v_{u}, v_{d}, v_{s}, \varphi_{s}, \varphi_{u},|\lambda|, \varphi_{\lambda},|\kappa|, \varphi_{\kappa},\left|A_{\kappa}\right| .
$$

Instead of using this set of original parameters it is convenient to convert it to a set of parameters which offer an intuitive interpretation. This is especially true for the parameters which can be replaced by gauge boson masses squared as they are measurable quantities. We have chosen the set,

$$
\underbrace{t_{h_{d}}, t_{h_{u}}, t_{h_{s}}, t_{a_{d}}, t_{a_{s}}, M_{H^{ \pm}}^{2}, M_{W}^{2}, M_{Z}^{2}, e}_{\text {on-shell }}, \underbrace{\tan \beta, v_{s}, \varphi_{s}, \varphi_{u},|\lambda|, \varphi_{\lambda},|\kappa|, \varphi_{\kappa},\left|A_{\kappa}\right|}_{\overline{\mathrm{DR}}}
$$

where the first part of the parameters are directly related to "physical" quantities ${ }^{9}$ and will be defined via on-shell conditions while the remaining parameters are understood as $\overline{\mathrm{DR}}$ parameters (see section 3.3.2). The transformation rules for going from set eq. (3.34) to set eq. (3.35) are given in appendix A.

\subsection{The Higgs boson sector at one-loop level}

At one-loop level, the Higgs boson sector and the corresponding relations between parameters of the theory and physical quantities are changed by radiative corrections. In

\footnotetext{
${ }^{9}$ Whether the tadpole parameters can be called physical quantities is debatable but certainly their introduction is motivated by physical interpretation. Therefore, in a slight abuse of the language, we are also calling the renormalization conditions for the tadpole parameters on-shell.
} 
particular, the Higgs boson mass matrix receives contributions from the renormalized selfenergies ${ }^{10} \hat{\Sigma}_{i j}\left(p^{2}\right)$ at an external momentum squared $p^{2}$,

$$
\hat{\Sigma}_{i j}\left(p^{2}\right)=\Sigma_{i j}\left(p^{2}\right)+\frac{1}{2} p^{2}\left[\delta \mathcal{Z}^{\dagger}+\delta \mathcal{Z}\right]_{i j}-\frac{1}{2}\left[\delta \mathcal{Z}^{\dagger} \mathcal{D}_{H}+\mathcal{D}_{H}^{\dagger} \delta \mathcal{Z}\right]_{i j}-\left[\mathcal{R} \delta \mathcal{M}_{\Phi \Phi} \mathcal{R}^{\dagger}\right]_{i j},(3.3
$$

with $i, j=1, \ldots, 6$ and $H_{6}=G$ the Goldstone boson. The unrenormalized self-energies $\Sigma_{i j}$ are obtained by taking into account all possible contributions to the Higgs boson self-energy, including the ones from fermion, gauge boson, Goldstone boson, Higgs boson, chargino, neutralino, sfermion and ghost loops.

The wave function renormalization matrix $\delta \mathcal{Z}$ in the basis of the Higgs boson mass eigenstates is derived via rotation from the corresponding matrix $\delta \mathcal{Z}_{\Phi}$ in the basis of the Higgs boson states $\Phi$,

$$
\delta \mathcal{Z}=\mathcal{R} \delta \mathcal{Z}_{\Phi} \mathcal{R}^{\dagger}
$$

The Higgs boson fields $\Phi$ are renormalized by replacing the fields by renormalized ones and a renormalization factor. This can be expressed as, valid up to one-loop order, with the field renormalization constant $\delta \mathcal{Z}_{\Phi}$ as

$$
\Phi \rightarrow\left(1+\frac{1}{2} \delta \mathcal{Z}_{\Phi}\right) \Phi
$$

where $\delta \mathcal{Z}_{\Phi}=\mathcal{R}^{G} \delta \mathcal{Z}_{\phi} \mathcal{R}^{G^{\dagger}}$. The field renormalization constant $\delta \mathcal{Z}^{\phi}$ of the interaction eigenstates $\phi$ is a diagonal matrix

$$
\delta \mathcal{Z}_{\phi}=\operatorname{diag}\left(\delta Z_{H_{u}}, \delta Z_{H_{d}}, \delta Z_{S}, \delta Z_{H_{d}}, \delta Z_{H_{u}}, \delta Z_{S}\right)
$$

The explicit definitions and expressions for $\delta Z_{H_{u}}, \delta Z_{H_{d}}$ and $\delta Z_{S}$ are given in section 3.3.1.

The matrix $\delta \mathcal{M}_{\Phi \Phi}$ denotes the counterterm matrix in the basis of the Higgs boson states $\Phi$ which has been introduced within the renormalization procedure by replacing the parameters in the mass matrix as given in appendix A in eqs. (A.1)-(A.8) by their renormalized ones and corresponding counterterms and expanding about the renormalized parameters. The part linear in the counterterms forms the mass matrix counterterm. The specific definitions of the parameters and the determination of the counterterms are discussed in section 3.3.2.

\subsubsection{Higgs boson field renormalization}

The field renormalization constants introduced in eq. (3.39) are defined in the $\overline{\mathrm{DR}}$ scheme. The precise expressions for $\delta Z_{H_{d}}, \delta Z_{H_{u}}$ and $\delta Z_{S}$ are determined via the following system of equations

$$
\begin{aligned}
& \delta Z_{H_{d}}\left(\left|\mathcal{R}_{i 1}\right|^{2}+\left|\mathcal{R}_{i 4} \sin \beta+\mathcal{R}_{i 6} \cos \beta\right|^{2}\right)+\delta Z_{H_{u}}\left(\left|\mathcal{R}_{i 2}\right|^{2}+\left|\mathcal{R}_{i 4} \cos \beta-\mathcal{R}_{i 6} \sin \beta\right|^{2}\right) \\
& \quad+\delta Z_{S}\left(\left|\mathcal{R}_{i 3}\right|^{2}+\left|\mathcal{R}_{i 5}\right|^{2}\right)=-\left.\Sigma_{i i}^{\prime}\right|_{\text {div }} \quad \text { with } \quad i=1,2,3
\end{aligned}
$$

\footnotetext{
${ }^{10}$ In general, we call $\hat{\Sigma}$ and $\Sigma$ renormalized and unrenormalized self-energy, respectively.
} 
where

$$
\Sigma_{i i}^{\prime}=\left.\frac{\partial \Sigma_{i i}\left(p^{2}\right)}{\partial p^{2}}\right|_{p^{2}=\left(M_{H_{i}}^{(0)}\right)^{2}} .
$$

The subscript 'div' denotes that only the divergent parts proportional to $\Delta$ are taken into account with $\Delta=2 /(4-D)-\gamma_{E}+\ln 4 \pi$ and $\gamma_{E}$ being the Euler constant and $D$ the number of dimensions. The pole of $\Delta$ for $D=4$ characterizes the divergences. Solving this system of equations eq. (3.40) yields

$$
\begin{aligned}
\delta Z_{H_{d}} & =\frac{1}{r}\left[\left(r_{23} r_{32}-r_{22} r_{33}\right) \Sigma_{11}^{\prime}+\left(r_{12} r_{33}-r_{13} r_{32}\right) \Sigma_{22}^{\prime}+\left(r_{13} r_{22}-r_{12} r_{23}\right) \Sigma_{33}^{\prime}\right]_{\mathrm{div}}, \\
\delta Z_{H_{u}} & =\frac{1}{r}\left[\left(r_{21} r_{33}-r_{23} r_{31}\right) \Sigma_{11}^{\prime}+\left(r_{13} r_{31}-r_{11} r_{33}\right) \Sigma_{22}^{\prime}+\left(r_{11} r_{23}-r_{13} r_{21}\right) \Sigma_{33}^{\prime}\right]_{\mathrm{div}}, \\
\delta Z_{S} & =\frac{1}{r}\left[\left(r_{22} r_{31}-r_{21} r_{32}\right) \Sigma_{11}^{\prime}+\left(r_{11} r_{32}-r_{12} r_{31}\right) \Sigma_{22}^{\prime}+\left(r_{12} r_{21}-r_{11} r_{22}\right) \Sigma_{33}^{\prime}\right]_{\mathrm{div}},
\end{aligned}
$$

with

$$
\begin{aligned}
r_{i 1} & =\left(\left|\mathcal{R}_{i 1}\right|^{2}+\left|\mathcal{R}_{i 4} \sin \beta+\mathcal{R}_{i 6} \cos \beta\right|^{2}\right), \\
r_{i 2} & =\left(\left|\mathcal{R}_{i 2}\right|^{2}+\left|\mathcal{R}_{i 4} \cos \beta-\mathcal{R}_{i 6} \sin \beta\right|^{2}\right), \\
r_{i 3} & =\left(\left|\mathcal{R}_{i 3}\right|^{2}+\left|\mathcal{R}_{i 5}\right|^{2}\right), \\
r & =r_{11} r_{22} r_{33}+r_{12} r_{23} r_{31}+r_{13} r_{32} r_{21}-r_{11} r_{23} r_{32}-r_{13} r_{22} r_{31}-r_{12} r_{21} r_{33} .
\end{aligned}
$$

It should be noted that $\mathcal{R}_{i 6}=0$ for $i \neq 6$ and hence, in eqs. (3.45) and (3.46) terms proportional to $\mathcal{R}_{i 6}$ vanish for the values $i=1,2,3$ needed in eqs. (3.42)-(3.44).

\subsubsection{Parameter renormalization}

The parameter renormalization is performed by replacing the parameters by the renormalized ones and the corresponding counterterms,

$$
\begin{array}{rlrl}
t_{\phi} & \rightarrow t_{\phi}+\delta t_{\phi} & \text { with } \quad \phi=\left\{h_{d}, h_{u}, h_{s}, a_{d}, a_{s}\right\}, \\
M_{H^{ \pm}}^{2} & \rightarrow M_{H^{ \pm}}^{2}+\delta M_{H^{ \pm}}^{2}, & M_{W}^{2} \rightarrow M_{W}^{2}+\delta M_{W}^{2}, \quad M_{Z}^{2} \rightarrow M_{Z}^{2}+\delta M_{Z}^{2}, \\
e & \rightarrow\left(1+\delta Z_{e}\right) e, & & \\
\tan \beta & \rightarrow \tan \beta+\delta \tan \beta, & v_{s} \rightarrow v_{s}+\delta v_{s}, \\
\varphi_{s} & \rightarrow \varphi_{s}+\delta \varphi_{s}, & \varphi_{u} & \rightarrow \varphi_{u}+\delta \varphi_{u}, \\
\lambda & \rightarrow \lambda+\delta \lambda=\lambda+e^{i \varphi_{\lambda}} \delta|\lambda|+i \lambda \delta \varphi_{\lambda}, \quad \kappa \rightarrow \kappa+\delta \kappa=\kappa+e^{i \varphi_{\kappa}} \delta|\kappa|+i \kappa \delta \varphi_{\kappa}, \\
\left|A_{\kappa}\right| & \rightarrow\left|A_{\kappa}\right|+\delta\left|A_{\kappa}\right| .
\end{array}
$$

In the case of complex parameters the complex counterterms can be understood in terms of two real counterterms, one for the absolute value and one for the phase, as in eq. (3.54) for $\delta \lambda$ and $\delta \kappa$.

As we make use of the chargino and the neutralino sector for the determination of the counterterms $\delta v_{s}, \delta \varphi_{s}, \delta \lambda, \delta \kappa$ and $\delta \varphi_{u}$ we also need to renormalize the gaugino mass 
parameters $M_{1}$ and $M_{2}$,

$$
\begin{aligned}
& M_{1} \rightarrow M_{1}+\delta M_{1}=M_{1}+e^{i \varphi_{M_{1}}} \delta\left|M_{1}\right|+i M_{1} \delta \varphi_{M_{1}}, \\
& M_{2} \rightarrow M_{2}+\delta M_{2}=M_{2}+e^{i \varphi_{M_{2}}} \delta\left|M_{2}\right|+i M_{2} \delta \varphi_{M_{2}} .
\end{aligned}
$$

To keep the relations as general as possible we do not make use of the $R$-symmetry relations here and keep both gaugino mass parameters complex.

In the following, we list all the renormalization conditions and counterterms. The renormalization scheme applied here is a generalization of the "mixed scheme" of ref. [127] for complex parameters - we will be brief on the conditions that can be directly taken from ref. [127].

(i-v) Tadpole parameters:

The renormalization conditions for the tadpole parameters are chosen such that the linear terms of the Higgs boson fields in the Higgs potential also vanish at one-loop level,

$$
\delta t_{\phi}=T_{\phi} \quad \text { with } \quad \phi=h_{d}, h_{u}, h_{s}, a_{d}, a_{s},
$$

where $T_{\phi}$ denotes the contribution of the irreducible one-loop tadpole diagrams.

(vi - viii) Masses of the gauge bosons and the charged Higgs boson:

The masses of the gauge bosons and of the charged Higgs boson are determined via on-shell conditions requiring that the mass parameters squared correspond to the pole masses squared leading to

$$
\delta M_{W}^{2}=\widetilde{\operatorname{Re}} \Sigma_{W W}^{T}\left(M_{W}^{2}\right), \quad \delta M_{Z}^{2}=\widetilde{\operatorname{Re}} \Sigma_{Z Z}^{T}\left(M_{Z}^{2}\right), \quad \delta M_{H^{ \pm}}^{2}=\widetilde{\operatorname{Re}} \Sigma_{H^{\mp} H^{ \pm}}\left(M_{H^{ \pm}}^{2}\right),
$$

where $\Sigma_{W W}^{T}$ and $\Sigma_{Z Z}^{T}$ are the transverse parts of the unrenormalized $W$ boson and $Z$ boson self-energy, respectively, while $\Sigma_{H^{\mp} H^{ \pm}}$denotes the unrenormalized self-energy of the charged Higgs boson. $\widetilde{R e}$ takes only the real part of the scalar loop functions but keeps the complex structure of the parameters.

(ix) Electric charge:

The electric charge is fixed via the $e \bar{e} \gamma$ vertex in such a way that this vertex does not receive any corrections at the one-loop level in the Thomson limit, i.e. for zero momentum transfer. This yields (cf. ref. [132] up to a different sign convention in the second term)

$$
\delta Z_{e}=\frac{1}{2} \Sigma_{\gamma \gamma}^{T^{\prime}}(0)+\frac{s_{\theta_{W}}}{c_{\theta_{W}} M_{Z}^{2}} \Sigma_{\gamma Z}^{T}(0)
$$

with $\Sigma_{\gamma \gamma}^{T}$ and $\Sigma_{\gamma Z}^{T}$ being the transverse part of the unrenormalized photon self-energy and the unrenormalized mixing of photon and $Z$ boson, respectively. 
(x) Ratio of the vacuum expectation values $\tan \beta$ :

The ratio of the vacuum expectation values $\tan \beta$ is defined as a $\overline{\mathrm{DR}}$ parameter and the counterterm is given by [133-139]

$$
\delta \tan \beta=\left.\frac{\tan \beta}{2}\left[\delta Z_{H_{u}}-\delta Z_{H_{d}}\right]\right|_{\text {div }} .
$$

(xi,xii) Vacuum expectation value $v_{s}$ and phase $\varphi_{s}$ :

The singlet vacuum expectation value $v_{s}$ and the phase $\varphi_{s}$ are determined as $\overline{\mathrm{DR}}$ parameters. For the derivation of the corresponding counterterms, we start out from the on-shell conditions for the chargino masses,

$$
\left.\widetilde{\operatorname{Re}} \hat{\Sigma}_{\chi_{i i}^{+}}(p) \tilde{\chi}_{i}^{+}(p)\right|_{p^{2}=m_{\chi_{i}^{ \pm}}^{2}}=0, \quad i=1,2,
$$

where $\hat{\Sigma}_{\chi_{i i}^{+}}$are the renormalized chargino self-energies. Applying the decomposition of the fermionic self-energy

$$
\hat{\Sigma}_{i j}\left(p^{2}\right)=\not p \hat{\Sigma}_{i j}^{L}\left(p^{2}\right) \mathcal{P}_{L}+\not p \hat{\Sigma}_{i j}^{R}\left(p^{2}\right) \mathcal{P}_{R}+\hat{\Sigma}_{i j}^{L s}\left(p^{2}\right) \mathcal{P}_{L}+\hat{\Sigma}_{i j}^{R s}\left(p^{2}\right) \mathcal{P}_{R}
$$

with $\mathcal{P}_{L, R}=\left(1 \mp \mp \gamma_{5}\right) / 2$ being the left- and right-handed projectors, leads to the finite relations

$$
\begin{aligned}
& {\left[m_{\tilde{\chi}_{i}^{ \pm}}\left(\widetilde{\operatorname{Re}} \hat{\Sigma}_{\chi_{i i}^{+}}^{L}\left(p^{2}\right)+\widetilde{\operatorname{Re}} \hat{\Sigma}_{\chi_{i i}^{+}}^{R}\left(p^{2}\right)\right)+\widetilde{\operatorname{Re}} \hat{\Sigma}_{\chi_{i i}^{+}}^{L s}\left(p^{2}\right)+\widetilde{\operatorname{Re}} \hat{\Sigma}_{\chi_{i i}^{+}}^{R s}\left(p^{2}\right)\right]_{p^{2}=m_{\chi_{i}^{ \pm}}^{2}}=0,} \\
& {\left[m_{\tilde{\chi}_{i}^{ \pm}}\left(\widetilde{\operatorname{Re}} \hat{\Sigma}_{\chi_{i i}^{+}}^{L}\left(p^{2}\right)-\widetilde{\operatorname{Re}} \hat{\Sigma}_{\chi_{i i}^{+}}^{R}\left(p^{2}\right)\right)-\widetilde{\operatorname{Re}} \hat{\Sigma}_{\chi_{i i}^{+}}^{L s}\left(p^{2}\right)+\widetilde{\operatorname{Re}} \hat{\Sigma}_{\chi_{i i}^{+}}^{R s}\left(p^{2}\right)\right]_{p^{2}=m_{\chi_{i}^{+}}^{2}}=0,}
\end{aligned}
$$

which can be exploited for the determination of the counterterms. Using the expressions given in eqs. (C.1)-(C.4) in appendix C for the renormalized self-energies yields

$$
\begin{aligned}
& \left.\operatorname{Re}\left(U^{*} \delta M_{C} V^{\dagger}\right)\right|_{\operatorname{div}} \\
& \quad=\left.\frac{1}{2}\left[m_{\tilde{\chi}_{i}^{ \pm}}\left(\Sigma_{\chi_{i i}^{+}}^{L}\left(p^{2}\right)+\Sigma_{\chi_{i i}^{+}}^{R}\left(p^{2}\right)\right)+\Sigma_{\chi_{i i}^{+}}^{L s}\left(p^{2}\right)+\Sigma_{\chi_{i i}^{+}}^{R s}\left(p^{2}\right)\right]_{p^{2}=m_{\chi_{i}^{ \pm}}^{2}}\right|_{\operatorname{div}} \\
& \quad=: \operatorname{Re} \delta m_{\chi_{i i}^{+}}, \\
& \left.\operatorname{Im}\left(U^{*} \delta M_{C} V^{\dagger}\right)\right|_{\operatorname{div}} \\
& =\left.\frac{i}{2}\left[\Sigma_{\chi_{i i}^{+}}^{R s}\left(p^{2}\right)-\Sigma_{\chi_{i i}^{+}}^{L s}\left(p^{2}\right)+i m_{\tilde{\chi}_{i}^{ \pm}}\left(U^{*} \operatorname{Im} \delta Z_{R}^{C} U^{T}+V \operatorname{Im} \delta Z_{L}^{C} V^{\dagger}\right)_{i i}\right]_{p^{2}=m_{\chi_{i}^{+}}^{2}}\right|_{\operatorname{div}} \\
& \quad=: \operatorname{Im} \delta m_{\chi_{i i}^{+}} .
\end{aligned}
$$

The imaginary parts of the field renormalization constants have been set to zero, hence $\operatorname{Im} \delta Z_{R}^{C}=\operatorname{Im} \delta Z_{L}^{C}=0$. With

$$
\delta M_{C}=\left(\begin{array}{cc}
\delta M_{2} & \sqrt{2} e^{-i \varphi_{u}}\left[\delta\left(M_{W} s_{\beta}\right)-i s_{\beta} M_{W} \delta \varphi_{u}\right] \\
\sqrt{2} \delta\left(c_{\beta} M_{W}\right) & \frac{e^{i \varphi_{s}}}{\sqrt{2}}\left[\lambda \delta v_{s}+i \lambda v_{s} \delta \varphi_{s}+v_{s} \delta \lambda\right]
\end{array}\right),
$$


solving eqs. (3.66) and (3.67) for $\delta v_{s}+i v_{s} \delta \varphi_{s}$ and $\delta M_{2},{ }^{11}$ we obtain

$$
\begin{aligned}
\delta M_{2}= & \frac{1}{\left|U_{11}\right|^{2}-\left|V_{12}\right|^{2}}\left[V_{11} U_{11} \delta m_{\chi_{11}^{+}}-V_{21} U_{21} \delta m_{\chi_{22}^{+}}\right. \\
& \left.-\left.U_{11} U_{12}^{*} \delta M_{C_{21}}\right|_{\text {div }}-\left.V_{11} V_{12}^{*} \delta M_{C_{12}}\right|_{\text {div }}\right], \\
\delta v_{s}+i v_{s} \delta \varphi_{s}= & \frac{\sqrt{2} \lambda^{*} e^{-i \varphi_{s}}}{|\lambda|^{2}\left(\left|U_{11}\right|^{2}-\left|V_{12}\right|^{2}\right)}\left[-V_{12} U_{12} \delta m_{\chi_{11}^{+}}+V_{22} U_{22} \delta m_{\chi_{22}^{+}}\right. \\
& \left.+\left.U_{11}^{*} U_{12} \delta M_{C_{12}}\right|_{\text {div }}+\left.V_{11}^{*} V_{12} \delta M_{C_{21}}\right|_{\text {div }}\right]-v_{s} \lambda^{*} \frac{\delta \lambda}{|\lambda|^{2}} .
\end{aligned}
$$

It should be noted that $\delta \varphi_{u}$ contained in $\delta M_{C_{12}}$ as well as $\delta \lambda$ have not been defined yet. We need additional conditions given by eqs. (3.88)-(3.90). Together with eqs. (3.69) and (3.70) they form a system of linear equations that can be easily solved but leads to lengthy expressions.

(xiii-xvii) Couplings $\lambda$ and $\kappa$ and the phase $\varphi_{u}$ :

The phase $\varphi_{u}$ as well as $\lambda$ and $\kappa$ are also defined as $\overline{\mathrm{DR}}$ parameters. On-shell conditions for the neutralino masses ${ }^{12}$

$$
\left.\widetilde{\operatorname{Re}} \hat{\Sigma}_{\chi_{i i}^{0}}(p) \tilde{\chi}_{i}^{0}(p)\right|_{p^{2}=m_{\chi_{i}^{0}}^{2}}=0, \quad i=1, \ldots, 4
$$

are exploited to derive the following finite relations, ${ }^{13}$

$$
\begin{aligned}
& {\left[m_{\tilde{\chi}_{i}^{0}}\left(\widetilde{\operatorname{Re}} \hat{\Sigma}_{\chi_{i i}^{0}}^{L}\left(p^{2}\right)+\widetilde{\operatorname{Re}} \hat{\Sigma}_{\chi_{i i}^{0}}^{R}\left(p^{2}\right)\right)+\widetilde{\operatorname{Re}} \hat{\Sigma}_{\chi_{i i}^{0}}^{L s}\left(p^{2}\right)+\widetilde{\operatorname{Re}} \hat{\Sigma}_{\chi_{i i}^{0}}^{R s}\left(p^{2}\right)\right]_{p^{2}=m_{\chi_{i}^{0}}^{2}}=0,} \\
& {\left[m_{\tilde{\chi}_{i}^{0}}\left(\widetilde{\operatorname{Re}} \hat{\Sigma}_{\chi_{i i}^{0}}^{L}\left(p^{2}\right)-\widetilde{\operatorname{Re}} \hat{\Sigma}_{\chi_{i i}^{0}}^{R}\left(p^{2}\right)\right)-\widetilde{\operatorname{Re}} \hat{\Sigma}_{\chi_{i i}^{0}}^{L s}\left(p^{2}\right)+\widetilde{\operatorname{Re}} \hat{\Sigma}_{\chi_{i i}^{0}}^{R s}\left(p^{2}\right)\right]_{p^{2}=m_{\chi_{i}^{0}}^{2}}=0,}
\end{aligned}
$$

with $i=1, \ldots, 4$. Applying the eqs. (C.8)-(C.11) in appendix C leads to

$$
\begin{aligned}
\left.\operatorname{Re}\left(\mathcal{N}^{*} \delta M_{N} \mathcal{N}^{\dagger}\right)_{i i}\right|_{\operatorname{div}} & =\left.\left[m_{\tilde{\chi}_{i}^{0}} \Sigma_{\chi_{i i}^{0}}^{L}\left(p^{2}\right)+\frac{1}{2}\left(\Sigma_{\chi_{i i}^{0}}^{L s}\left(p^{2}\right)+\Sigma_{\chi_{i i}^{0}}^{R s}\left(p^{2}\right)\right)\right]_{p^{2}=m_{\chi_{i}^{0}}^{2}}\right|_{\text {div }}=: \operatorname{Re} \delta m_{\chi_{i i}^{0}}, \\
\left.\operatorname{Im}\left(\mathcal{N}^{*} \delta M_{N} \mathcal{N}^{\dagger}\right)_{i i}\right|_{\operatorname{div}} & =\left.\frac{i}{2}\left[\Sigma_{\chi_{i i}^{0}}^{R s}\left(p^{2}\right)-\Sigma_{\chi_{i i}^{0}}^{L s}\left(p^{2}\right)+2 i m_{\tilde{\chi}_{i}^{0}}\left(\mathcal{N}^{*} \operatorname{Im} \delta Z^{N} \mathcal{N}^{\dagger}\right)_{i i}\right]_{p^{2}=m_{\chi_{i}^{0}}^{2}}\right|_{\operatorname{div}} \\
& =: \operatorname{Im} \delta m_{\chi_{i i}^{0}}
\end{aligned}
$$

$i=1, \ldots, 4$ where already $\Sigma_{\chi_{i i}^{0}}^{L}=\Sigma_{\chi_{i i}^{0}}^{R}$ has been used which is true due to the Majorana character of the neutralinos. The imaginary part of $\delta Z^{N}$ has been set to zero, $\operatorname{Im} \delta Z^{N}=0$. The elements of the mass matrix counterterm $\delta M_{N}$ are derived as

$$
\delta M_{N_{11}}=\delta M_{1}
$$

\footnotetext{
${ }^{11}$ Even though $M_{2}$ does not enter the Higgs boson sector at tree-level, it has to be dealt with due to its entanglement in eq. (3.68).

${ }^{12}$ In terms of an on-shell scheme, if both chargino masses were defined on-shell only three of the neutralino masses could be chosen independently. Although eq. (3.71) leads to two independent equations for each $i$, for $i=4$ it is only partly used to fix one still undefined phase.

${ }^{13}$ It should be noted that, in general, both eqs. (3.72) and (3.73) for $i=4$ only hold for the divergent part simultaneously as all the parameters are already fixed by other conditions.
} 


$$
\begin{aligned}
& \delta M_{N_{22}}=\delta M_{2} \\
& \delta M_{N_{55}}=\sqrt{2} e^{i \varphi_{s}}\left[v_{s} \delta \kappa+\kappa \delta v_{s}+i \kappa v_{s} \delta \varphi_{s}\right] \\
& \delta M_{N_{13}}=-M_{Z} s_{\theta_{W}} c_{\beta}^{2} s_{\beta} \delta \tan \beta+\frac{c_{\beta}}{2 s_{\theta_{W}} M_{Z}}\left[\delta M_{W}^{2}-\delta M_{Z}^{2}\right] \\
& \delta M_{N_{14}}=e^{-i \varphi_{u}}\left[\delta\left(s_{\beta} M_{Z} s_{\theta_{W}}\right)-i s_{\beta} M_{Z} s_{\theta_{W}} \delta \varphi_{u}\right] \\
& \delta M_{N_{23}}=\delta\left(c_{\beta} M_{W}\right) \\
& \delta M_{N_{24}}=-e^{-i \varphi_{u}}\left[\delta\left(s_{\beta} M_{W}\right)-i s_{\beta} M_{W} \delta \varphi_{u}\right] \\
& \delta M_{N_{34}}=-\frac{1}{\sqrt{2}} e^{i \varphi_{s}}\left[v_{s} \delta \lambda+\lambda \delta v_{s}+i \lambda v_{s} \delta \varphi_{s}\right] \\
& \delta M_{N_{35}}=-\sqrt{2} e^{i \varphi_{u}}\left[\lambda \frac{\delta\left(s_{\beta} M_{W} s_{\theta_{W}}\right)}{e}-\frac{s_{\beta} M_{W} s_{\theta_{W}}}{e}\left(\lambda \delta Z_{e}-\delta \lambda-i \lambda \delta \varphi_{u}\right)\right] \\
& \delta M_{N_{45}}=-\sqrt{2}\left[\lambda \frac{\delta\left(c_{\beta} M_{W} s_{\theta_{W}}\right)}{e}+\frac{c_{\beta} M_{W} s_{\theta_{W}}}{e}\left(\delta \lambda-\lambda \delta Z_{e}\right)\right] \\
& \delta M_{N_{33}}=\delta M_{N_{44}}=\delta M_{N_{12}}=\delta M_{N_{15}}=\delta M_{N_{25}}=0 .
\end{aligned}
$$

As the neutralino mass matrix is symmetric, this also holds for the counterterm mass matrix and therefore $\delta M_{N_{i j}}=\delta M_{N_{j i}}$. Rewriting eqs. (3.74) and (3.75) explicitly and solving for $\delta M_{1}$ results in the following set of equations,

$$
\begin{aligned}
& \delta M_{1}=\frac{\mathcal{N}_{11}^{2}}{\left|\mathcal{N}_{11}\right|^{4}}\left[\delta m_{\chi_{11}^{0}}-2 \mathcal{N}_{11}^{*}\left[\mathcal{N}_{13}^{*} \delta M_{N_{13}}+\mathcal{N}_{14}^{*} \delta M_{N_{14}}\right]-2 \mathcal{N}_{12}^{*}\left[\mathcal{N}_{13}^{*} \delta M_{N_{23}}+\mathcal{N}_{14}^{*} \delta M_{N_{24}}\right]\right. \\
& -2 \mathcal{N}_{13}^{*}\left[\mathcal{N}_{14}^{*} \delta M_{N_{34}}+\mathcal{N}_{15}^{*} \delta M_{N_{35}}\right]-2 \mathcal{N}_{14}^{*} \mathcal{N}_{15}^{*} \delta M_{N_{45}} \\
& -\left(\mathcal{N}_{12}^{*}\right)^{2} \delta M_{2}-\left(\mathcal{N}_{15}^{*}\right)^{2} \delta M_{N_{55}}, \\
& 2\left[a_{214} \delta M_{N_{14}}+a_{224} \delta M_{N_{24}}+2 a_{234} \delta M_{N_{34}}+a_{235} \delta M_{N_{35}}+a_{245} \delta M_{N_{45}}\right] \\
& +a_{222} \delta M_{2}+a_{255} \delta M_{N_{55}} \\
& =\left(\mathcal{N}_{21}^{*}\right)^{2} \delta m_{\chi_{11}^{0}}-\left(\mathcal{N}_{11}^{*}\right)^{2} \delta m_{\chi_{22}^{0}}-2 a_{213} \delta M_{N_{13}}-2 a_{223} \delta M_{N_{23}} \\
& 2\left[a_{314} \delta M_{N_{14}}+a_{324} \delta M_{N_{24}}+2 a_{334} \delta M_{N_{34}}+a_{335} \delta M_{N_{35}}+a_{345} \delta M_{N_{45}}\right] \\
& +a_{322} \delta M_{2}+a_{355} \delta M_{N_{55}} \\
& =\left(\mathcal{N}_{31}^{*}\right)^{2} \delta m_{\chi_{11}^{0}}-\left(\mathcal{N}_{11}^{*}\right)^{2} \delta m_{\chi_{33}^{0}}-2 a_{313} \delta M_{N_{13}}-2 a_{323} \delta M_{N_{23}}, \\
& \operatorname{Im}\left\{2\left[a_{414} \delta M_{N_{14}}+a_{424} \delta M_{N_{24}}+2 a_{434} \delta M_{N_{34}}+a_{435} \delta M_{N_{35}}+a_{445} \delta M_{N_{45}}\right]\right. \\
& \left.+a_{422} \delta M_{2}+a_{455} \delta M_{N_{55}}\right\} \\
& =\operatorname{Im}\left\{\left(\mathcal{N}_{41}^{*}\right)^{2} \delta m_{\chi_{11}^{0}}-\left(\mathcal{N}_{11}^{*}\right)^{2} \delta m_{\chi_{44}^{0}}-2 a_{413} \delta M_{N_{13}}-2 a_{423} \delta M_{N_{23}}\right\},
\end{aligned}
$$

where we have introduced the shorthand notation

$$
a_{i j k}=\left(\mathcal{N}_{i 1}^{*}\right)^{2} \mathcal{N}_{1 j}^{*} \mathcal{N}_{1 k}^{*}-\left(\mathcal{N}_{11}^{*}\right)^{2} \mathcal{N}_{i j}^{*} \mathcal{N}_{i k}^{*}
$$

As stated above, taking eqs. (3.88)-(3.90) together with eqs. (3.69) and (3.70) leads to a system of equations with 4 complex and one real equation linear in the counterterms that has to be solved for $\delta M_{2}, \delta v_{s}, \delta \varphi_{s}, \delta \lambda, \delta \kappa$ and $\delta \varphi_{u}$. 
(xviii) Absolute value of the singlet trilinear coupling $\left|A_{\kappa}\right|$ :

The absolute value of the singlet trilinear coupling $\left|A_{\kappa}\right|$ is determined as a $\overline{\mathrm{DR}}$ parameter. The corresponding counterterm is calculated using

$$
\mathcal{R}_{i 5} \mathcal{R}_{j 5} \hat{\Sigma}_{i j}\left(M_{a_{s} a_{s}}\right)=0
$$

which is equivalent to

$$
\delta M_{a_{s} a_{s}}=\mathcal{R}_{i 5} \mathcal{R}_{j 5} \Sigma_{i j}\left(M_{a_{s} a_{s}}\right),
$$

with $\delta M_{a_{s} a_{s}}$ depending on $\delta\left|A_{\kappa}\right|$. Dropping the finite parts and solving for $\delta\left|A_{\kappa}\right|$ yields

$$
\begin{aligned}
& \delta\left|A_{\kappa}\right|=-\frac{\sqrt{2}}{|\kappa| v_{s}\left[3 c_{\varphi_{z}}-3 t_{\left.\varphi_{z} \frac{f_{2}}{\left|A_{\kappa}\right|}\right]}\right.}\left\{\mathcal{R}_{i 5} \mathcal{R}_{j 5} \Sigma_{i j}\left(M_{a_{s}, a_{s}}\right)-\delta f_{1}\right. \\
& \left.+\frac{3}{\sqrt{2}}\left|A_{\kappa}\right|\left[v_{s} c_{\varphi_{z}} \delta|\kappa|+|\kappa| c_{\varphi_{z}} \delta v_{s}\right]+\frac{3}{\sqrt{2}}|\kappa| v_{s} t_{\varphi_{z}} \delta f_{2}\right\}_{\mathrm{div}}
\end{aligned}
$$

with

$$
\begin{aligned}
f_{1}= & {\left[M_{H^{ \pm}}^{2}-M_{W}^{2} c_{\Delta \beta}^{2}\right] \frac{M_{W}^{2} s_{\theta_{W}}^{2} s_{2 \beta}^{2}}{e^{2} v_{s}^{2} c_{\Delta \beta}^{2}}-\frac{M_{W} s_{\theta_{W}} s_{2 \beta} c_{\beta} c_{\beta_{B}}^{2}}{e v_{s}^{2} c_{\Delta \beta}^{2}}\left[t_{h_{u}}+t_{\beta} t_{\beta_{B}}^{2} t_{h_{d}}\right]+\frac{t_{h_{s}}}{v_{s}} } \\
& +|\lambda| M_{W}^{2} \frac{s_{\theta_{W}}^{2} s_{2 \beta}}{e^{2} v_{s}^{2}}\left[2|\lambda| M_{W}^{2} \frac{s_{\theta_{W}}^{2}}{e^{2}} s_{2 \beta}+3|\kappa| v_{s}^{2} c_{\varphi_{y}}\right] \\
f_{2}= & \frac{\sqrt{2}}{v_{s}}\left(\frac{2 M_{W} s_{\theta_{W}} c_{\beta}}{e|\kappa| v_{s}^{2}} t_{a_{d}}+\frac{3|\lambda| M_{W}^{2} s_{\theta_{W}}^{2} s_{2 \beta}}{e^{2}} s_{\varphi_{y}}-\frac{1}{|\kappa| v_{s}} t_{a_{s}}\right) .
\end{aligned}
$$

The counterterms $\delta f_{1}$ and $\delta f_{2}$, which are functions of counterterms of the parameters defined as input in eq. (3.35), are determined by replacing the parameters by renormalized ones plus corresponding counterterms and expanding about the parameters. It has to be taken into account that in the expressions $\Delta \beta=\beta-\beta_{B}$ only $\beta$ is treated within the renormalization procedure. The angle $\beta_{B}=\beta_{c}=\beta_{n}$ is the mixing angle of the charged Higgs bosons and the angle extracting the Goldstone boson defined in appendix A.

Following the approach above, it has been found that the counterterms $\delta \varphi_{s}, \delta \varphi_{\lambda}, \delta \varphi_{\kappa}$, $\delta \varphi_{u}, \delta \varphi_{M_{1}}$ and $\delta \varphi_{M_{2}}$ vanish. In that respect, it is interesting to note that eqs. (3.67) and (3.75) allow for a certain freedom of choice; some potentially divergent parts can be moved into $\operatorname{Im} \delta Z_{L}^{C}, \operatorname{Im} \delta Z_{R}^{C}$ and $\operatorname{Im} \delta Z^{N}$ which do not appear in the calculation of the Higgs boson masses as the charginos and neutralinos only enter through internal lines in the Feynman diagrams.

The derivation of the counterterms for $v_{s}, \varphi_{s}, \lambda, \kappa$ and $\varphi_{u}$ presented above is not unique. In a second approach on-shell conditions for all the neutralino masses plus an additional condition from the chargino sector, eq. (3.67) for $i=1$, have been exploited to calculate the $\overline{\mathrm{DR}}$ counterterms leading to the same result and providing a good cross-check. A further possibility is to determine the counterterms within the Higgs boson sector only. We have also done that but this does not test the calculation at the same level as using conditions from the chargino and neutralino sector. 


\subsection{Loop corrected Higgs boson masses and mixing matrix elements}

The one-loop corrected scalar Higgs boson masses squared are extracted numerically as the zeroes of the determinant of the two-point vertex functions $\hat{\Gamma}$,

$$
\hat{\Gamma}\left(p^{2}\right)=i\left(1 \cdot p^{2}-\mathcal{M}^{11}\right) \quad \text { with } \quad\left(\mathcal{M}^{11}\right)_{i j}=\left(M_{H_{i}}^{(0)}\right)^{2} \delta_{i j}-\hat{\Sigma}_{i j}\left(p^{2}\right) \quad i, j=1, \ldots, 5,
$$

where $\hat{\Sigma}_{i j}\left(p^{2}\right)$ is given in eq. (3.36). The superscript $1 l$ denotes the one-loop order.

Starting from eq. (3.97) the Higgs masses at one-loop level can be obtained via an iterative procedure. ${ }^{14}$ To calculate the one-loop mass of the $n^{\text {th }}$ Higgs boson the external momentum squared $p^{2}$ in the renormalized self-energies $\hat{\Sigma}_{i j}$ is set equal to the tree-level mass squared $\left(p^{2}=\left(M_{H_{n}}^{(0)}\right)^{2}\right)$ in the first iteration step. Then, the mass matrix part of $\hat{\Gamma}$, i.e. $\mathcal{M}^{11}$, is diagonalized. The thus obtained $n^{\text {th }}$ eigenvalue is the first approximation of the squared one-loop mass. In the next iteration step $p^{2}$ is set equal to this value and once again the eigenvalues of $\mathcal{M}^{11}$ are calculated to yield the next approximation of the one-loop mass. This iteration procedure is repeated until a precision of $10^{-9}$ is reached. All five Higgs boson masses are calculated this way.

Note that in eq. (3.97) the mixing with the Goldstone bosons is not taken into account but we have checked numerically that the effect is negligible. Furthermore, it was shown in ref. [140], that in the MSSM it is sufficient to include the mixing with the Goldstone boson, whereas the mixing with the longitudinal component of the $Z$ boson does not have to be added explicitly. Taking into account the mixing of the Goldstone boson as well as the mixing of the $Z$ boson leads to the same result as only including the Goldstone boson mixing.

Due to the radiative corrections, not only the masses of the particles receive contributions but at the same time the tree-level mass eigenstates mix to form new one-loop mass eigenstates. In order to take this into account the Higgs mixing matrix $\mathcal{R}$ which performs the rotation from the interaction eigenstates to the mass eigenstates has to be adjusted so that

$$
H_{i}^{1 l}=\mathcal{R}_{i j}^{1 l} \Phi_{j}
$$

In the numerical analysis, for the simplicity of the notation we drop the superscript $1 l$ again. If not explicitly mentioned one-loop corrections are included.

The rotation of the tree-level to the one-loop mass eigenstates could be obtained by calculating finite wave function correction factors. The procedures to calculate these for a $2 \times 2$ or $3 \times 3$ mass matrix are described in ref. [79] and need to be extended for the $5 \times 5$ case. Another option is to apply the $p^{2}=0$ approximation. After setting the momenta in $\mathcal{M}^{11}$ to zero, the rotation matrix that relates the tree-level to the one-loop mass eigenstates can be defined as the matrix that diagonalizes $\mathcal{M}^{11}$. The latter procedure

\footnotetext{
${ }^{14}$ This procedure is not strictly of one-loop order. It was shown, however, in ref. [139] for the MSSM that this procedure gives much exacter values for the Higgs mass including implicitly higher order corrections than a strict treatment at one-loop level.
} 
has the advantage that the mixing matrix is unitary. The drawback is that it does not retain the full momentum dependence. For our numerical analysis we used the $p^{2}=0$ approximation for the determination of the mixing matrix. But we checked numerically that the differences between both methods are negligible.

\section{$4 \quad$ Numerical analysis}

The calculation of the one-loop corrected Higgs boson masses has been performed in two different calculations. While in one calculation the Feynman rules have been derived from the NMSSM Lagrangian and implemented in a FeynArts model file [141-143], they have been obtained with the Mathematica package SARAH [144-146] in the second calculation and cross-checked against the first calculation. The self-energies and tadpoles have been evaluated with the help of FormCalc $[147,148]$ in the 't Hooft-Feynman gauge. The divergent integrals, regularized in the constrained differential renormalization scheme [149], have been computed numerically with LoopTools [147, 148]. For the evaluation of the counterterms, numerical diagonalization of the one-loop corrected Higgs boson mass matrix and the determination of the mass eigenvalues finally two independent Mathematica programs have been written.

We follow the SUSY Les Houches Accord (SLHA) [150-152] and compute the parameters $M_{W}^{2}$ and $e$ of our input set defined in eq. (3.35) from the SLHA pre-defined input values for the Fermi constant $G_{F}=1.16637 \cdot 10^{-5} \mathrm{GeV}^{-2}$, the $Z$ boson mass $M_{Z}=91.187 \mathrm{GeV}$ and the electroweak coupling $\alpha=1 / 137$. If not stated otherwise, we use the running $\overline{\mathrm{DR}}$ top quark mass $m_{t}$ at a common scale $Q=\sqrt{m_{Q_{3}} m_{t_{R}}}$. It is obtained from the top quark pole mass $M_{t}=173.2 \mathrm{GeV}$ by taking the routines of NMSSMTools [153-156]. In the same way we obtain the running $\overline{\mathrm{DR}}$ bottom quark mass starting from the SLHA input value $m_{b}\left(m_{b}\right)^{\overline{\mathrm{MS}}}=4.19 \mathrm{GeV}$. For the light quarks we chose $m_{u}=2.5 \mathrm{MeV}, m_{c}=1.27 \mathrm{GeV}$, $m_{d}=4.95 \mathrm{MeV}, m_{s}=101 \mathrm{MeV}$ [157] and for the $\tau$ mass $m_{\tau}=1.777 \mathrm{GeV}$.

In the following we exemplify the effects of complex phases in the one-loop corrections to the NMSSM Higgs boson masses in different scenarios. We require the scenarios to be compatible with the recent results of the LHC Higgs boson searches [1-17] in the limit of the real NMSSM, i.e. for vanishing CP-violating phases. ${ }^{15}$ Our starting points are the NMSSM benchmark points presented in ref. [60] which have been slightly modified for our analysis. With not too heavy stop masses and not too substantial mixing they avoid unnaturally large finetuning, and $\lambda$ and $\kappa$ have been chosen such that unitarity is not violated below the GUT scale. Furthermore, we paid attention to keep the effective $\mu \leq 200 \mathrm{GeV}$, with $\mu=\lambda v_{s} / \sqrt{2}$, in order not to violate tree-level naturalness. For each scenario we verified that the non SM-like Higgs bosons are not excluded by the searches at LEP [128, 129], Tevatron [130] and LHC. This has been cross-checked by running the program HiggsBounds $[158,159],{ }^{16}$ which needs the complex NMSSM Higgs couplings and branching ratios. The latter have

\footnotetext{
${ }^{15}$ Note, that this is only an arbitrary choice which was made for practical reasons. We could as well have demanded a complex NMSSM scenario to be compatible with the recent LHC searches.

${ }^{16}$ The program NMSSMTools [153-156] also performs these checks. It can be used, however, only for the case of the real NMSSM.
} 
been obtained by adapting the Fortran code HDECAY [169-174] to the complex NMSSM, in which we use the one-loop corrected Higgs boson masses and mixing matrix elements of our calculation. For the SM-like Higgs boson $H_{i}^{\text {SM-like }}$ of the real NMSSM we demand its mass to lie in the interval $120-130 \mathrm{GeV}$. Furthermore, the total significance for $H_{i}^{\text {SM-like }}$ should not deviate by more than $20 \%$ from the corresponding SM value. We roughly estimate the significance $S$ to be given by $S=N_{s} / \sqrt{N_{b}}$, where $N_{s}$ denotes the number of signal events and $N_{b}$ the number of background events. Hence our criteria for a scenario to be compatible with present LHC searches are

$$
\begin{aligned}
120 \mathrm{GeV} & \leq M_{H_{i}^{\mathrm{SM}-l i k e}} \leq 130 \mathrm{GeV} \\
S_{\mathrm{tot}}^{\mathrm{NMSSM}}\left(H_{i}^{\mathrm{SM}-\text { like }}\right) & =S_{\mathrm{tot}}^{\mathrm{SM}}\left(H^{\mathrm{SM}}\right) \pm 20 \% \quad \text { for } \quad M_{H^{\mathrm{SM}}}=M_{H_{i}^{\mathrm{SM}}} \text {-like } .
\end{aligned}
$$

In this case the scenario is estimated to be compatible with the present LHC searches taking into account experimental and theoretical uncertainties. We roughly approximate the total significance by adding in quadrature the significances of the various LHC Higgs search channels. We assume the number of background events to be the same both in the SM and the NMSSM case. For the calculation of the signal events we need the cross section values in the different channels, which we obtain as follows. We first calculate the inclusive production cross section by adding the gluon fusion, weak boson fusion, Higgsstrahlung and $t \bar{t}$ Higgs production cross sections. Associated production with $b \bar{b}$ does not play a role here, as the $\tan \beta$ values we chose are rather low. The gluon fusion value at NNLO QCD is obtained with HIGLU [160], which we have modified to the NMSSM case. Weak boson fusion and Higgs-strahlung at NLO QCD are computed with the programs VV2H and V2HV [161] by applying the modification factor due to the modified NMSSM Higgs coupling to gauge bosons compared to the SM case. Finally, the cross section value for $t \bar{t}$ Higgs production at NLO QCD [162-166] is obtained from the cross section values given at the LHC Higgs cross section working group webpage $[167,168]$ by applying the appropriate factor taking into account the change of the NMSSM Higgs Yukawa coupling with respect to the SM coupling. Note that the NLO QCD corrections are not affected by changes due to the NMSSM Higgs sector and can therefore readily be taken over from the SM case. The cross sections in the $W W, Z Z$ and $\gamma \gamma$ LHC search channels are obtained in the narrow width approximation by multiplication of the total cross section with the corresponding Higgs branching ratios into these final states. The branching ratios have been obtained from our modified Fortran code HDECAY [169-174], adapted to the complex NMSSM. The thus obtained cross sections for the various channels can be used to calculate the number of signal events. ${ }^{17}$ The experiments take into account QCD corrections beyond NLO and also electroweak corrections. As these are not available for the NMSSM we cannot take them into account here. They are of the order of a few percent depending on the process. Furthermore, ATLAS and CMS exploit more final states and combine them

\footnotetext{
${ }^{17}$ Note, that the luminosity factor in the calculation of the number of events and also the number of background events drop out in the comparison of the NMSSM case to the SM case, so that we only need to calculate the quadratic sum of the cross sections in the different final states for the NMSSM and for the SM and compare them.
} 
in a sophisticated statistical procedure, while we have taken into account only the most prominent ones. Our approximation should therefore be viewed only as a rough estimate, good enough though to eliminate scenarios clearly excluded by the present LHC search results.

In all investigated scenarios we have taken the input values at the scale $Q=\sqrt{m_{Q_{3}} m_{t_{R}}}$. In order to comply with the present LHC searches [175-185], we have throughout taken the soft SUSY breaking mass parameters of the squarks of the first two generations equal to $1 \mathrm{TeV}$, and for simplicity also those of the sleptons. The corresponding trilinear couplings are taken to be $1 \mathrm{TeV}$. Furthermore, the right-handed soft SUSY breaking mass parameter of the sbottom sector is set equal to $1 \mathrm{TeV}$ and its trilinear coupling close to $1 \mathrm{TeV}$, so that we have

$$
\begin{aligned}
m_{U} & =m_{D}=m_{Q_{1,2}}=m_{E}=m_{L}=1 \mathrm{TeV} \\
A_{x} & =1 \mathrm{TeV} \quad(x=u, c, d, s, e, \mu, \tau) \\
A_{b} & \approx 1 \mathrm{TeV} .
\end{aligned}
$$

This leads to masses of $\sim 1 \mathrm{TeV}$ for the squarks of the first and second family, the sleptons and the heavier sbottom. Furthermore, all scenarios lead to the correct relic density in the limit of the real NMSSM, which has been checked with NMSSMTools which contains a link to MicrOMEGAs [186-189].

\subsection{Scenario with a SM-like $\boldsymbol{H}_{3}$}

The parameter set for this scenario is given by

$$
\begin{aligned}
|\lambda| & =0.72, \quad|\kappa|=0.20, \quad \tan \beta=3, \quad M_{H^{ \pm}}=629 \mathrm{GeV}, \quad\left|A_{\kappa}\right|=27 \mathrm{GeV}, \quad|\mu|=198 \mathrm{GeV} \\
\left|A_{b}\right| & =963 \mathrm{GeV}, \quad\left|A_{t}\right|=875 \mathrm{GeV}, \quad M_{1}=145 \mathrm{GeV}, \quad M_{2}=200 \mathrm{GeV}, \quad M_{3}=600 \mathrm{GeV}
\end{aligned}
$$

The slightly high values of $\lambda$ and $\kappa$ may require extra matter above the TeV scale [60]. ${ }^{18}$ We set all CP-violating phases to zero and subsequently turn on specific phases to study their respective influence. In this case, the signs of the tree-level CP-violating phases eqs. (3.18), (3.19) are then chosen as

$$
\operatorname{sign} \cos \varphi_{x}=+1, \quad \operatorname{sign} \cos \varphi_{z}=-1 .
$$

Furthermore, the left- and right-handed soft SUSY breaking mass parameters in the stop sector are given by $m_{Q_{3}}=490 \mathrm{GeV}$ and $m_{t_{R}}=477 \mathrm{GeV}$. This leads to relatively light stop masses $m_{\tilde{t}_{1}}=363 \mathrm{GeV}$ and $m_{\tilde{t}_{2}}=616 \mathrm{GeV}$, still allowed by the experiments [190-195]. ${ }^{19}$ In the calculation of the one-loop correction to the Higgs boson masses we have set the

\footnotetext{
${ }^{18}$ Being above the TeV scale it is not expected to influence LHC phenomenology, apart from the indirect effect of allowing $\lambda$ to be a somewhat larger than allowed by the usual perturbativity requirement in the NMSSM with no extra matter. If instead one accepts more finetuning in the theory and allows for higher stop masses, a Higgs mass of the order of $125 \mathrm{GeV}$ can be achieved for lower $\lambda$ values, cf. the discussion in section 4.2 .

${ }^{19}$ Note, that light stop masses and small mixing reduce the amount of finetuning [60].
} 
renormalization scale equal to $500 \mathrm{GeV}$, i.e. $\mu_{r e n}=500 \mathrm{GeV}$, if not stated otherwise. This scenario leads in the CP-conserving NMSSM to the one-loop corrected $H_{3}$ being SM-like with a mass $M_{H_{3}}=125 \mathrm{GeV}$ compatible with present LHC searches. In the following we discuss for various complex phase choices the phenomenology of the three lightest Higgs bosons. The two heavier ones receive mass corrections of maximally $2 \mathrm{GeV}$ leading to masses of $\sim 642 \mathrm{GeV}$ so that they are not excluded by present collider searches, with $H_{4}$ being mostly $\mathrm{CP}$-odd and $\mathrm{H}_{5}$ mostly $\mathrm{CP}$-even. We therefore do not display their masses explicitly.

\subsubsection{CP violation at tree-level}

As we have seen in section 3.1 a non-vanishing phase $\varphi_{y}$, cf. eq. (3.9), introduces CP violation at tree-level. Therefore $\mathrm{CP}$-even and $\mathrm{CP}$-odd Higgs mass eigenstates cannot be distinguished any more. A measure for $\mathrm{CP}$ violation concerning the state $H_{i}(i=1, \ldots, 5)$ is instead provided by the quantity

$$
r_{\mathrm{CP}}^{i} \equiv\left(\mathcal{R}_{i 1}\right)^{2}+\left(\mathcal{R}_{i 2}\right)^{2}+\left(\mathcal{R}_{i 3}\right)^{2},
$$

where $\mathcal{R}_{i j}$ are the matrix elements of the mixing matrix which diagonalizes the Higgs boson mass matrix, cf. eq. (3.29). A purely CP-even (CP-odd) mass eigenstate $H_{i}$ corresponds to $r_{\mathrm{CP}}^{i}=1(0)$. We first investigate the effect of a non-vanishing phase ${ }^{20}$

$$
\varphi_{\kappa} \neq 0
$$

The phases $\varphi_{A_{\lambda}}, \varphi_{A_{\kappa}}$ are fixed by the tadpole conditions eqs. (3.5), (3.7).

In figure 1 (left) we show the tree-level and one-loop masses of the two lightest Higgs mass eigenstates $H_{1,2}$ as a function of $\varphi_{\kappa}$, where $\varphi_{\kappa}=0$ corresponds to the real NMSSM. The phase is varied up to $\pi / 8$. Above this value it turns out that the phases $\varphi_{A_{\lambda}}$ and $\varphi_{A_{\kappa}}$ cannot be chosen in such a way that the tadpole conditions are fulfilled. The tree-level and one-loop corrected mass of the SM-like $H_{3}$ is shown in figure 1 (right). Figure 2 displays, as a function of $\varphi_{\kappa}$, the amount of CP violation $r_{\mathrm{CP}}^{i}$ (left) and the amount of the CP-even singlet component ${ }^{21}\left(\mathcal{R}_{i 3}\right)^{2}$ (right) for $H_{1,2,3}$. Finally figure 3 shows their coupling squared to the $V$ bosons $(V=Z, W)$ normalized to the SM as a function of $\varphi_{\kappa}$. As expected, the masses exhibit already at tree-level a sensitivity to the CP-violating phase $\varphi_{\kappa}$. In particular for the SM-like $H_{3}$ this dependence is more pronounced at one-loop level, changing its mass value by up to $9 \mathrm{GeV}$ for $\varphi_{\kappa} \in[0, \pi / 8]$. The one-loop correction increases the mass by $\sim 4$ to $11 \mathrm{GeV}$ depending on $\varphi_{\kappa}$ with larger mass values for larger CP-violating phases.

In the plots the grey areas are the parameter regions which are excluded due to the experimental constraints from LEP, Tevatron and LHC, and which have been obtained with HiggsBounds. ${ }^{22}$ This is the case for $0.074 \pi<\varphi_{\kappa}<0.099 \pi$ and $\varphi_{\kappa}>0.112 \pi$. The dashed region excludes the parameter regions where the criteria stated in eq. (4.2) of

\footnotetext{
${ }^{20}$ The choice of non-vanishing $\varphi_{\kappa}$ allows to investigate mixing effects of the Higgs bosons while suppressing the phase relevant for the neutral electric dipole moment [116].

${ }^{21}$ The CP-odd singlet component is given by $\left(\mathcal{R}_{i 5}\right)^{2}$.

${ }^{22}$ The exclusion is due to the LEP constraint on $H_{1}$ from the Higgs boson search in the $Z b \bar{b}$ final state stemming from a Higgs boson produced in Higgs-strahlung with subsequent decay into a $b$-quark pair.
} 

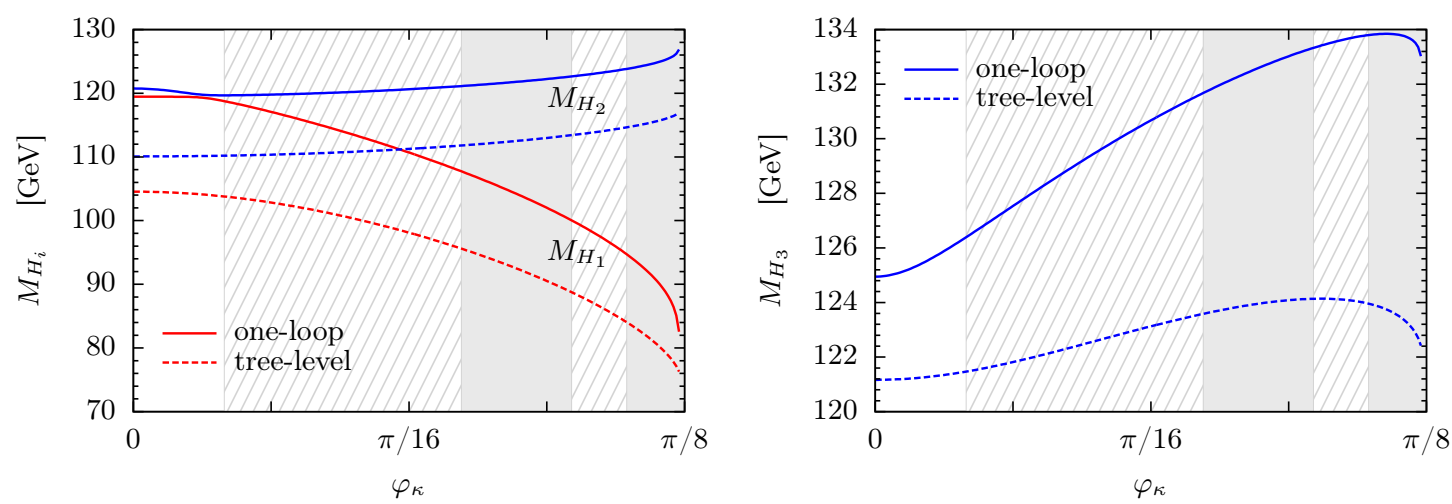

Figure 1. Left: Tree-level (dashed) and one-loop corrected (full) Higgs boson masses for $H_{1}$ (red) and $H_{2}$ (blue) as a function of $\varphi_{\kappa}$. Right: Tree-level (dashed) and one-loop (full) mass $M_{H_{3}}$ as a function of $\varphi_{\kappa}$. The exclusion region due to LEP, Tevatron and LHC data is shown as grey area, the region with the SM-like Higgs boson not being compatible with an excess of data around $125 \mathrm{GeV}$ as dashed area.
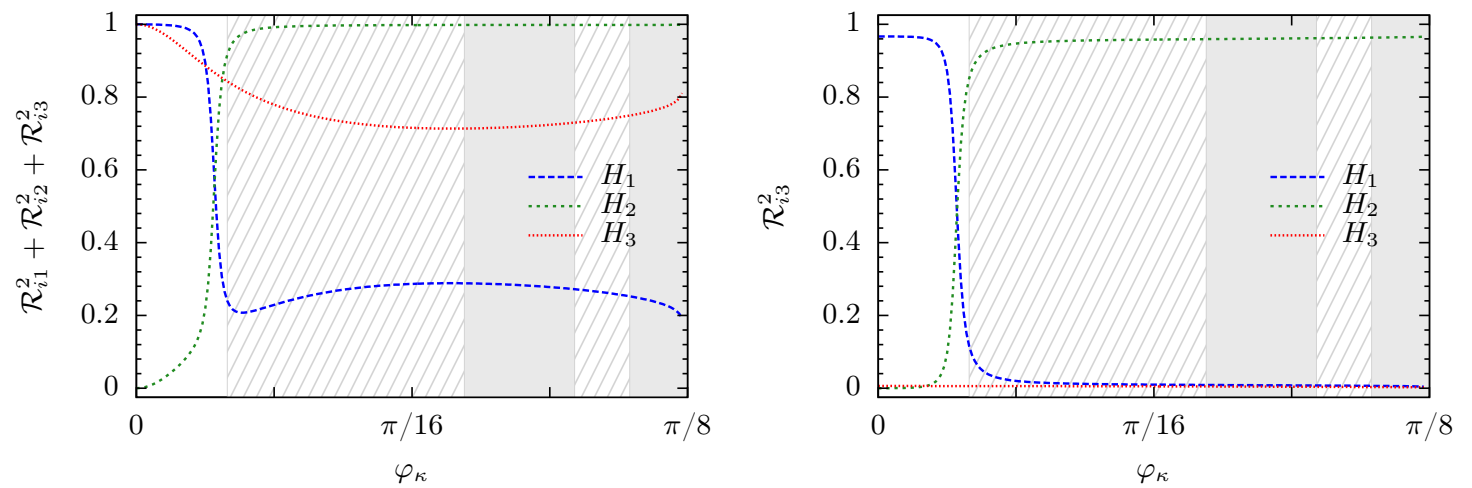

Figure 2. The amount of $\mathrm{CP}$ violation $r_{\mathrm{CP}}^{i}$ for $H_{i}(i=1,2,3)$ as a function of $\varphi_{\kappa}$ (left). The amount of CP-even singlet component $\left(\mathcal{R}_{i 3}\right)^{2}$ as a function of $\varphi_{\kappa}$ (right).

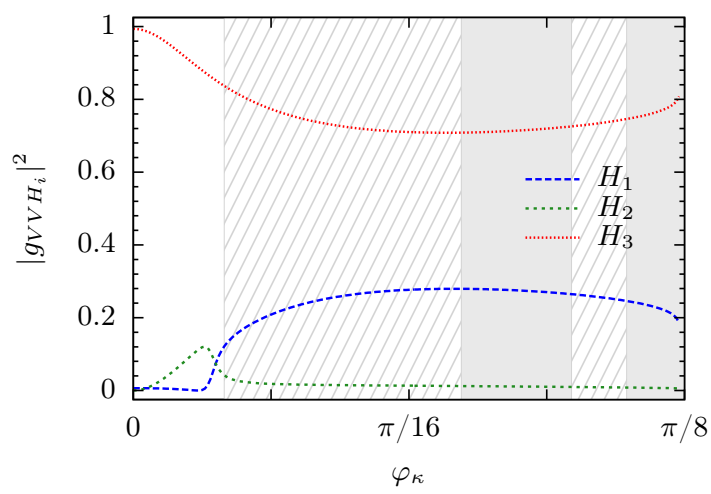

Figure 3. The $H_{i}$ coupling to $V(V=Z, W)$ bosons squared $(i=1,2,3)$ normalized to the SM coupling, $\left|g_{V V H_{i}}\right|^{2}$, as a function of $\varphi_{\kappa}$. 


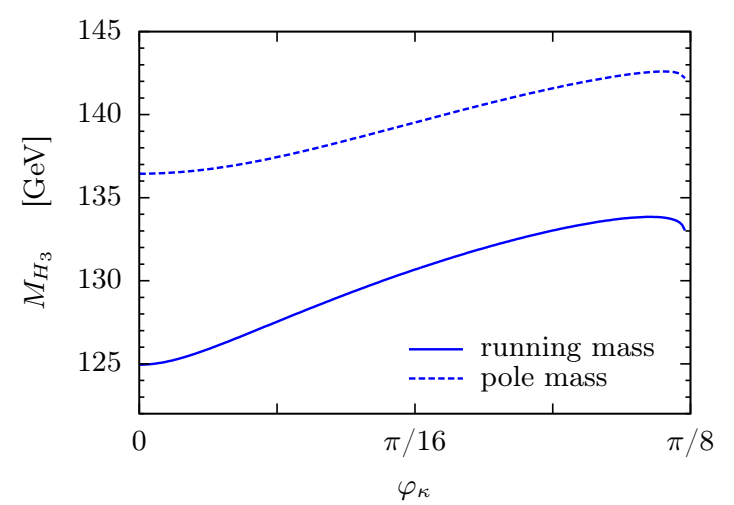

Figure 4. The one-loop corrected mass of the SM-like Higgs $H_{3}$ evaluated with the top and bottom running $\overline{\mathrm{DR}}$ masses (full) and with the corresponding pole masses (dashed).

compatibility with the recent Higgs excess around $125 \mathrm{GeV}$ cannot be fulfilled any more, here for $\varphi_{\kappa}>0.021 \pi$. The reason is that with increasing $\varphi_{\kappa}$ the eigenstate $H_{3}$ becomes more CP-odd and hence couples less to $V V(V=Z, W)$ as can be inferred from figure 2 (left) and figure 3 so that the total cross section becomes smaller and the significance deviates by more than $20 \%$ from the SM significance of a SM Higgs boson with same mass.

The one-loop corrections for the two lighter Higgs bosons $H_{1,2}$ increase their masses by $6-15 \mathrm{GeV}$ depending on $\varphi_{\kappa}$. The mass value $M_{H_{1}\left(H_{2}\right)}$ decreases (increases) with rising $\varphi_{\kappa}$. In the CP-conserving limit the one-loop masses of $H_{1,2}$ are $M_{H_{1}}=119.4 \mathrm{GeV}$ and $M_{H_{2}}=120.7 \mathrm{GeV}$, with $H_{1}$ being CP-even, cf. figure 2 (left), but CP-even singlet-like, cf. figure 2 (right), such that it hardly couples to SM particles and cannot be excluded by the experimental searches. The heavier Higgs $\mathrm{H}_{2}$ is dominantly CP-odd singlet-like (not plotted here) and is not excluded by the LEP, Tevatron and LHC searches due to both its singlet and its $\mathrm{CP}$-odd nature leading to a vanishing coupling to weak vector bosons, cf. figure 3. With increasing CP-violating phase the eigenstates $H_{1}$ and $H_{2}$ interchange their roles both with respect to their $\mathrm{CP}$ nature and their amount of CP-even singlet component, with the cross-over taking place at $\varphi_{\kappa} \approx \pi / 64$.

In order to get an estimate of the theoretical uncertainty due to the unknown higherorder corrections the one-loop corrections to the Higgs boson masses have been calculated with the top and bottom pole quark masses, $M_{t}=173.2 \mathrm{GeV}$ and $M_{b}=4.88 \mathrm{GeV}$, and compared to the results for the one-loop corrected masses evaluated with the running $\overline{\mathrm{DR}}$ top and bottom quark masses $m_{t, b}$ at the scale $Q=\sqrt{m_{Q_{3}} m_{t_{R}}}$. For our scenario they amount to $m_{t}=153.4 \mathrm{GeV}$ and $m_{b}=2.55 \mathrm{GeV}$. The result is shown in figure 4 . Whereas the slope of the curve hardly changes, the absolute values of the corrections change and are more important for a higher top quark mass. The theoretical uncertainty due to the different quark mass renormalization schemes can conservatively be estimated to $\sim 10 \%$.

\subsubsection{No tree-level CP violation}

We now keep the CP-violating phases $\varphi_{\kappa}$ and $\varphi_{\lambda}$ non-zero and vary them by the same amount, such that according to eq. (3.9) we have no tree-level CP-violating phase $\varphi_{y}$. In 

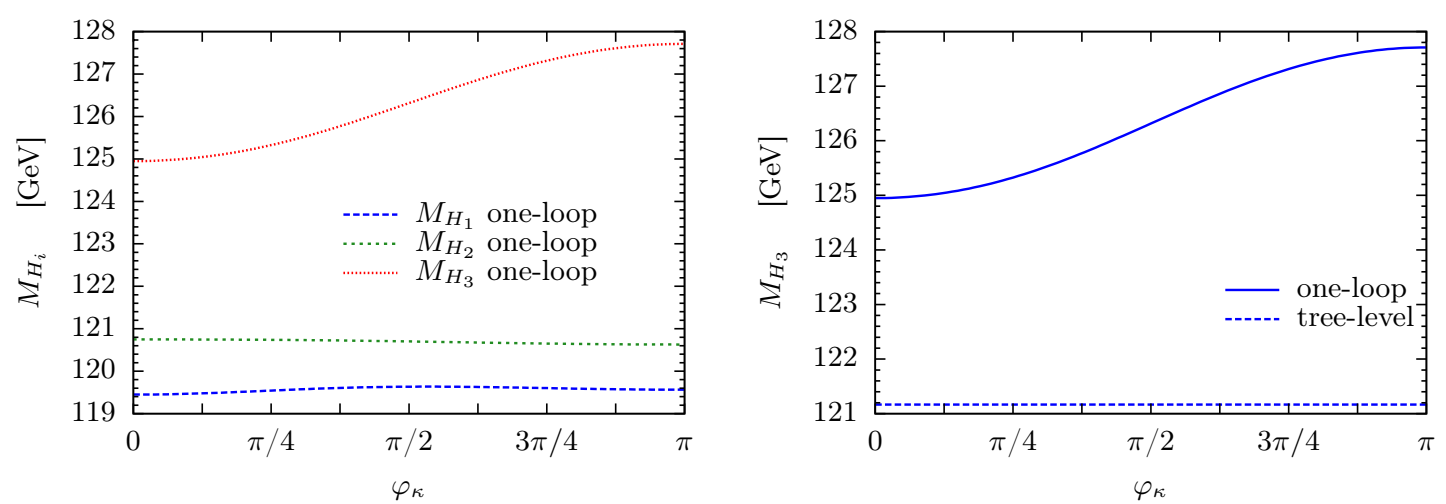

Figure 5. One-loop corrected Higgs boson masses $M_{H_{i}}(i=1,2,3)$ as a function of $\varphi_{\kappa}=\varphi_{\lambda}$ (left). Tree-level (dashed) and one-loop corrected (full) mass for $H_{3}$ as a function of $\varphi_{\kappa}=\varphi_{\lambda}$ (right).

the one-loop corrections $\varphi_{\kappa}, \varphi_{\lambda}$ enter separately so that CP violation is induced radiatively. Figure 5 (left) shows the one-loop corrected masses of the three lightest Higgs states $H_{1,2,3}$, figure 5 (right) compares the tree-level and one-loop corrected mass of the SM-like $H_{3}$, both as a function of $\varphi_{\kappa}$. The tree-level mass shows no dependence on $\varphi_{\kappa}$ as expected. The oneloop mass $M_{H_{3}}$ changes by only $\sim 3 \mathrm{GeV}$ for $\varphi_{\kappa}$ varying from 0 to $\pi$, and the loop-corrected masses for $H_{1,2}$ show almost no dependence on the CP-violating phase. The reason is that the dependence on the phase is due to the corrections from the stop sector which are the dominant contributions to the one-loop masses. The values of the stop masses change with the CP-violating phase. As $H_{3}$ has the largest $h_{u}$ component and hence couples more strongly to the up-type quarks it shows a stronger dependence on $\varphi_{\kappa}$ than $H_{1}$ and $H_{2}$. For $M_{H_{1}}\left(M_{H_{2}}\right)$ the mass corrections are of about 15 (11) GeV. With mass values around $120 \mathrm{GeV}$ they could lead to additional signals at the LHC if they were SM-like. However, due to the CP-odd nature of $H_{2}$, cf. figure 6 (left), it hardly couples to weak vector bosons. And the CP-even singlet character of $H_{1}$, compare with figure 6 (right), reduces its couplings to SM particles. These particles would therefore have considerably reduced signals at the LHC. The whole region over which $\varphi_{\kappa}=\varphi_{\lambda}$ are varied is hence still allowed by the LHC searches.

\subsubsection{Radiatively induced CP violation through the stop sector}

For completeness we investigate the case where only $\varphi_{A_{t}} \neq 0$. CP violation is thus only induced through loop corrections stemming from the stop sector. The one-loop corrected masses of $H_{1,2,3}$ are shown in figure 7 (left), the tree-level and one-loop corrected mass of the SM-like $H_{3}$ are displayed separately in figure 7 (right), both as function of $\varphi_{A_{t}}$. The tree-level masses of $H_{1,2}$ are increased by about $10-15 \mathrm{GeV}$ and their one-loop masses of $\sim 119.5$ and $121 \mathrm{GeV}$, respectively, hardly show any dependence on $\varphi_{A_{t}}$. The SM-like $H_{3}$ one-loop mass shows a small dependence varying by $\sim 2 \mathrm{GeV}$ for $\varphi_{A_{t}} \in[0, \pi]$, increasing the tree-level mass by 4-7 GeV. The reason is that $H_{3}$ has the largest $h_{u}$ component so that the dominant one-loop corrections stemming from the stop loops contribute more importantly to the radiative corrections of the Higgs mass matrix elements of $H_{3}$ than of $H_{1}$ and $H_{2}$. 

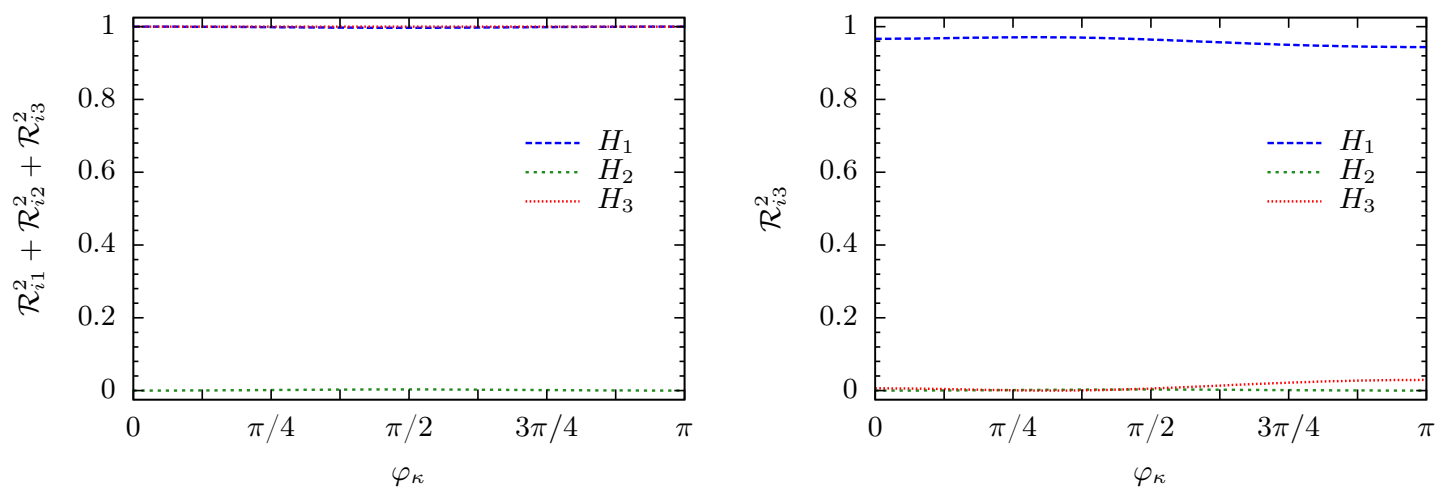

Figure 6. The amount of CP violation $r_{\mathrm{CP}}^{i}$ for $H_{i}(i=1,2,3)$ as a function of $\varphi_{\kappa}=\varphi_{\lambda}$ (left). The amount of CP-even singlet component $\left(\mathcal{R}_{i 3}\right)^{2}$ as a function of $\varphi_{\kappa}=\varphi_{\lambda}$ (right).
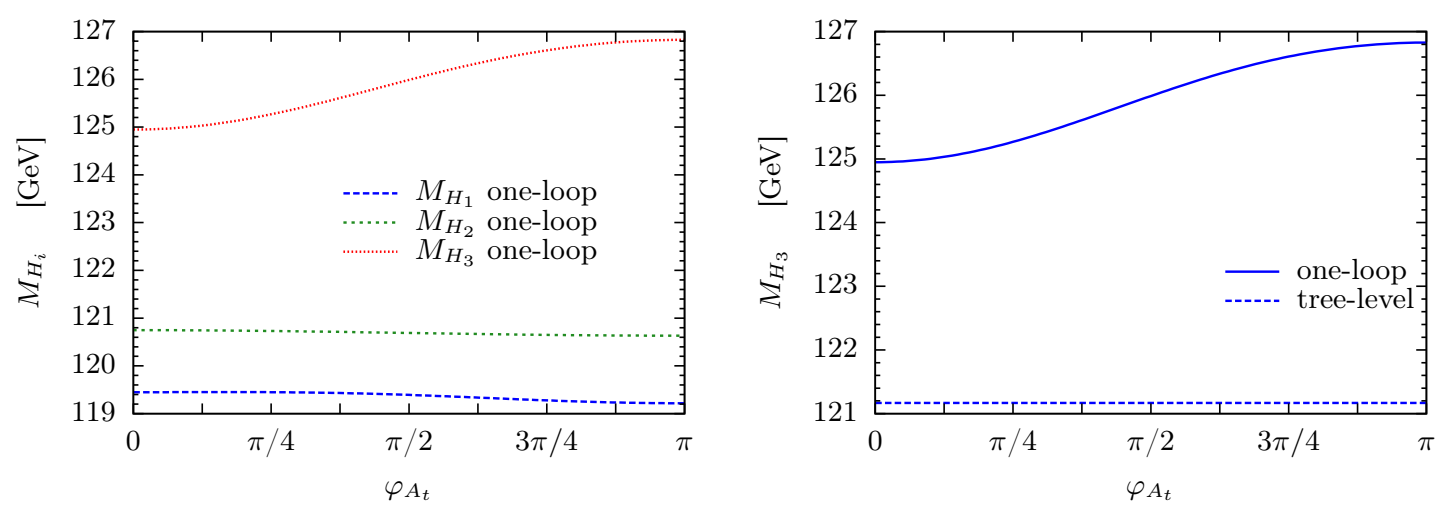

Figure 7. One-loop corrected Higgs boson masses $M_{H_{i}}(i=1,2,3)$ as a function of $\varphi_{A_{t}}$ (left). Tree-level (dashed) and one-loop (full) mass $M_{H_{3}}$ as a function of $\varphi_{A_{t}}$ (right).

The CP-even singlet nature of $H_{1}$, the CP-even character of $H_{1}$ and $H_{3}$ as well as the CP-odd one of $H_{2}$ are hardly affected by a change in $\varphi_{A_{t}}$ and are therefore not displayed here. As may have been expected, in this scenario loop-induced CP violation affects the phenomenology of the Higgs bosons less. Note, that the scenario is not excluded by LHC searches over the whole displayed phase range.

In figure 8 we investigate the theoretical error due to unknown higher-order corrections by varying the renormalization scale from $500 \mathrm{GeV}$ to half and twice the scale. The variation of the renormalization scale also changes the values of the input parameters and the running $\overline{\mathrm{DR}}$ top and bottom mass. For higher scales they become smaller and hence also the one-loop corrections to the masses decrease. The residual theoretical uncertainty can be estimated to about $4 \%$. We also checked the theoretical uncertainty due to the different quark mass renormalization schemes and found them to be of $\sim 10 \%$, hence of the same order as in the scenario studied in section 4.1.

In summary, the discussion of the various scenarios has shown that the impact of the CP-violating phase is crucial for the validity of the model. While a certain parameter set can still accommodate the experimental results for vanishing $\mathrm{CP}$ violation it may be 


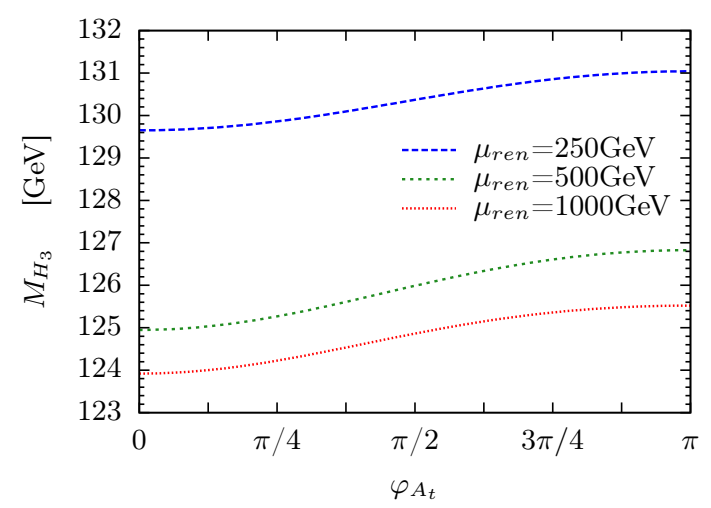

Figure 8. One-loop corrected Higgs boson masses $M_{H_{3}}$ as a function of $\varphi_{A_{t}}$ for three different renormalization scales, $\mu_{\text {ren }}=250$ (blue/long-dashed), 500 (green/short-dashed) and $1000 \mathrm{GeV}$ (red/dotted).

invalidated by non-vanishing CP phases. Turning this around, the experimental results will be useful to pin down the allowed amount of $\mathrm{CP}$ violation. The latter can arise from tree-level CP-violating phases in the Higgs sector or be radiatively induced. In the latter case the effects are found to be less pronounced. To get reliable predictions, the one-loop corrections have to be included as they not only considerably change the absolute mass values but also the singlet and CP-nature of the individual Higgs bosons as compared to the tree-level quantities.

\subsection{Scenario with SM-like $H_{1}$ or $H_{2}$}

The parameter set for this scenario, where, depending on the CP-violating phase, either $H_{1}$ or $H_{2}$ is SM-like, is given by

$$
\begin{array}{rlrlrl}
|\lambda| & =0.65, \quad|\kappa|=0.25, \quad \tan \beta=3, & M_{H^{ \pm}} & =619 \mathrm{GeV}, \quad\left|A_{\kappa}\right|=18 \mathrm{GeV}, & & |\mu|=199 \mathrm{GeV} \\
\left|A_{b}\right| & =971 \mathrm{GeV}, \quad\left|A_{t}\right|=1143 \mathrm{GeV}, \quad M_{1}=105 \mathrm{GeV}, & M_{2}=200 \mathrm{GeV}, & M_{3}=600 \mathrm{GeV} .
\end{array}
$$

The signs of the tree-level CP-violating phases eqs. (3.18) and (3.19) are

$$
\operatorname{sign} \cos \varphi_{x}=+1, \quad \operatorname{sign} \cos \varphi_{z}=-1
$$

The renormalization scale has been set to $\mu_{r e n}=650 \mathrm{GeV}$. The left- and right-handed soft SUSY breaking mass parameters in the stop sector $m_{Q_{3}}=642 \mathrm{GeV}$ and $m_{t_{R}}=632 \mathrm{GeV}$ lead to $m_{\tilde{t}_{1}}=514 \mathrm{GeV}$ and $m_{\tilde{t}_{2}}=768 \mathrm{GeV}$. The low value of $\lambda$ respects the bounds imposed by unitarity [60]. We allow for tree-level CP violation by choosing $\varphi_{\kappa} \neq 0$. The remaining complex phases are all set to zero, except for $\varphi_{A_{\lambda}}$ and $\varphi_{A_{\kappa}}$ which follow from the tadpole conditions eqs. (3.5), (3.7).

The tree-level and and one-loop corrected masses of $H_{1}$ and $H_{2}$ are shown in figure 9 (left), as a function of $\varphi_{\kappa}$. Beyond $\varphi_{\kappa} \approx 0.1 \pi$ the tadpole conditions are not fulfilled any more. The corresponding couplings squared to weak vector bosons are plotted in figure 9 (right). The amount of $\mathrm{CP}$ violation of the three lightest Higgs bosons $H_{1,2,3}$ and their 

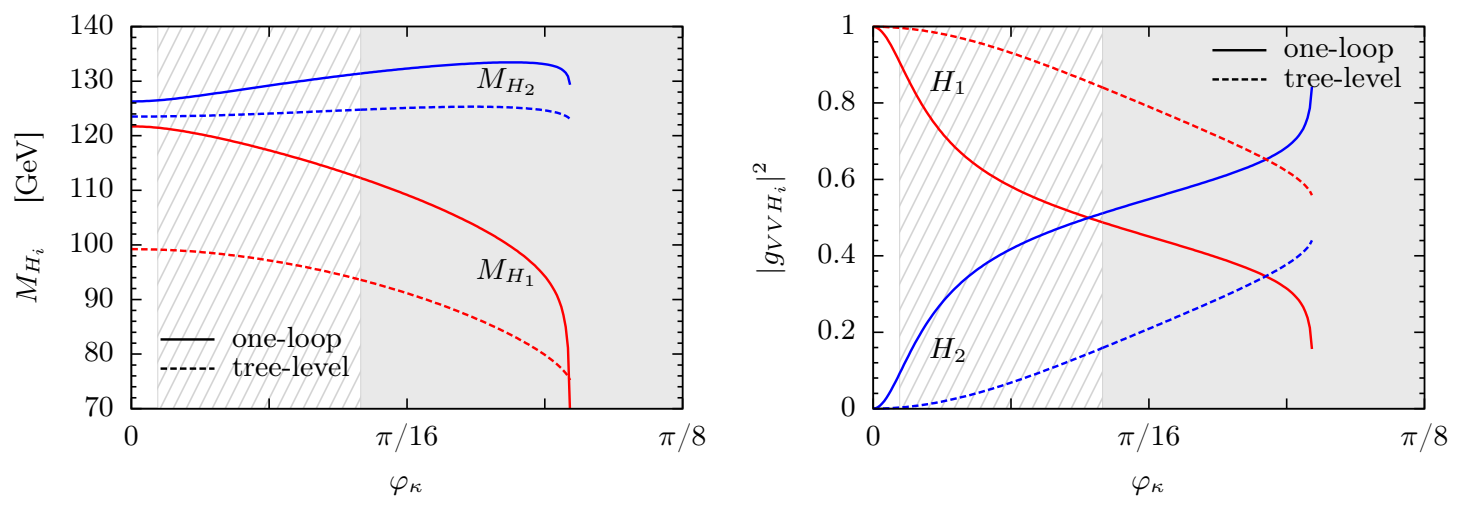

Figure 9. Left: Tree-level (dashed) and one-loop corrected (full) Higgs boson masses as a function of $\varphi_{\kappa}$ for $H_{1}$ (red) and $H_{2}$ (blue). Right: The $H_{1}$ (red) and $H_{2}$ (blue) Higgs couplings squared to two $V$ bosons $(V=W, Z)$ as a function of $\varphi_{\kappa}$ at tree-level (dashed) and at one-loop (full). The exclusion region due to LEP, Tevatron and LHC data is shown as grey area, the region with the SM-like Higgs boson not being compatible with an excess of data around $125 \mathrm{GeV}$ as dashed area.
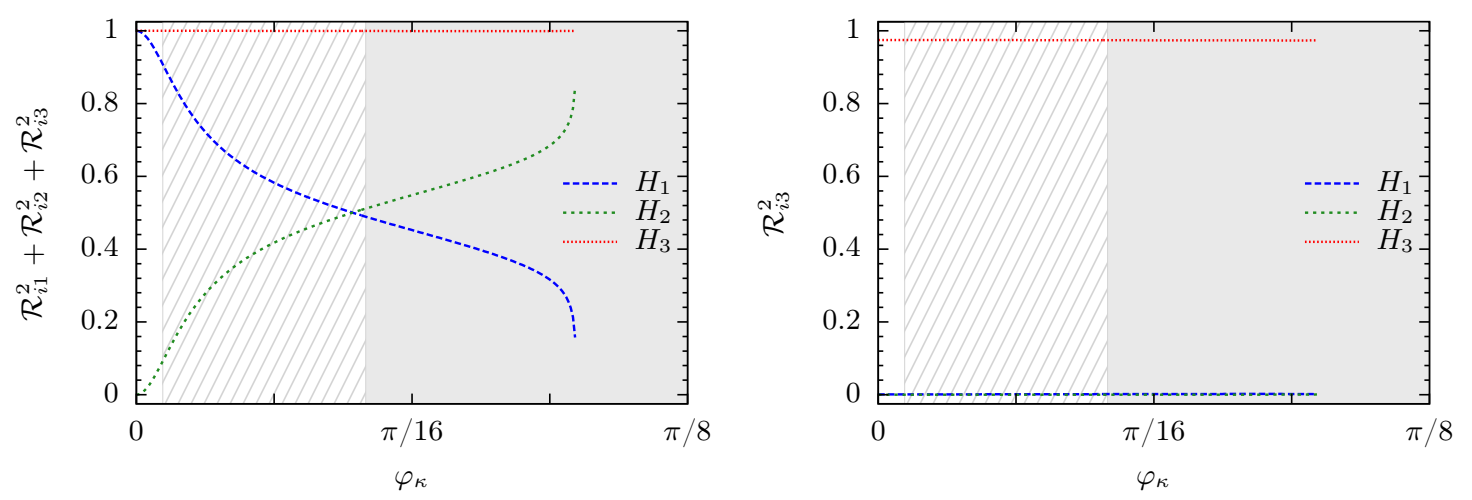

Figure 10. The amount of $\mathrm{CP}$ violation $r_{\mathrm{CP}}^{i}$ for $H_{i}(i=1,2,3)$ as a function of $\varphi_{\kappa}$ (left). The amount of CP-even singlet component $\left(\mathcal{R}_{i 3}\right)^{2}$ as a function of $\varphi_{\kappa}$ (right).

CP-even singlet component are displayed in figure 10 (left) and (right), respectively, as a function of $\varphi_{\kappa}$. As can be inferred from the figures, in the limit of the real NMSSM $H_{1}$ is $\mathrm{CP}$-even and has SM-like couplings while $\mathrm{H}_{2}$ is CP-odd. The heavier $\mathrm{H}_{3}$ is CP-even over the whole $\varphi_{\kappa}$ range. The CP-even singlet components of $H_{1}$ and $H_{2}$ vanish, cf. figure 10 (right). However, $H_{2}$ is CP-odd singlet-like. This is reflected in the couplings of $H_{1}$ and $H_{2}$ to the weak vector bosons. The one-loop corrections for $\varphi_{\kappa}=0$ shift the $H_{1}$ mass from 99 to about $122 \mathrm{GeV}$ so that its mass is compatible with the excess observed at the LHC. The mass of the second Higgs boson $H_{2}$ is increased by about $3 \mathrm{GeV}$ to $126 \mathrm{GeV}$ and could have been observed at the LHC, if its coupling to gauge bosons were not suppressed due to its CP-odd character so that it is not excluded by the present experimental constraints from the LHC. Furthermore, for $\varphi_{\kappa}=0$ the total significance concerning $H_{1}$ is compatible with LHC searches according to our criteria eq. (4.2). 
The scenario is interesting because with increasing $\varphi_{\kappa}$ the $\mathrm{CP}$ character of $H_{1,2}$ changes rapidly (with a cross-over at $\varphi_{\kappa} \approx 3 \pi / 64$ where $H_{1,2}$ interchange their roles with $H_{1}$ being more $\mathrm{CP}$-odd like and $\mathrm{H}_{2}$ more $\mathrm{CP}$-even like). This dependence on the $\mathrm{CP}$ violating phase is at one-loop more pronounced than at tree-level and makes that already beyond $\varphi_{\kappa} \approx 0.006 \pi H_{1}$ cannot fulfill the role of the SM-like Higgs boson any more as its couplings deviate too much from the SM case to fulfill the requirement of eq. (4.2). On the other hand, the $H_{2}$ couplings are not yet SM-like and once this is the case $H_{2}$ is already too heavy to be compatible with LHC searches. Hence, above $\varphi_{\kappa} \approx 0.006 \pi$ the scenario does not comply with the criteria of eq. (4.2) any more and it is excluded as indicated by the dashed region. Beyond $\varphi_{\kappa} \approx 0.052 \pi$ the grey region shows that the searches at LEP invalidate this parameter choice due to the LEP limit on $H_{1}$ in $Z b \bar{b}$. Therefore a large portion of this scenario is likely to be excluded, constraining $\varphi_{\kappa}$ to be almost zero and hence a real NMSSM.

This scenario illustrates particularly well the importance of the one-loop corrections and the impact on the restriction of a possible CP-violating phase. Firstly, the one-loop corrections are crucial to shift the mass of the SM-like Higgs boson to a mass value which is compatible with the excess observed at the LHC. However, the one-loop corrections also amplify the dependence on the CP-violating phase of both the Higgs masses and in particular the mixing matrix elements and hence the coupling to the weak vector bosons. Neglecting for the moment for the sake of this discussion the fact that at tree-level $H_{1}$ does not fulfill the mass constraint, the restriction of the $\mathrm{CP}$-violating phase due to deviations from the SM significance would be less severe at tree-level than at one-loop level due to the smooth tree-level dependence on the CP-violating phase. The one-loop corrections are hence crucial to correctly define parameter scenarios which are compatible with present LHC searches and to derive the correct exclusion limits for scenarios dropping out of this constraint.

The CP-even $H_{3}$, which has been not shown explicitly in all plots, is dominantly CP-even singlet-like. Its tree-level mass of $\sim 148-152 \mathrm{GeV}$ for $\varphi_{\kappa}$ increasing from 0 to $\sim 3 \pi / 16$ receives one-loop corrections of $\sim 3-4 \mathrm{GeV}$. The one-loop corrections show the same dependence on $\varphi_{\kappa}$ as the tree-level mass. Due to its singlet character at present it cannot be excluded by LHC searches.

We close our numerical analysis by a few general remarks on the identification of $\mathrm{CP}$ violating effects in Higgs boson phenomenology. Although the Higgs mass values and the Higgs couplings are changed by the inclusion of $\mathrm{CP}$ violation this is no unique indicator of CP violation. Contrary to the MSSM where only in the complex case e.g. there are parameter ranges where a light Higgs boson is not excluded by LEP due to a suppressed coupling to $Z$ bosons, in the NMSSM such a suppression can also arise in case of vanishing $\mathrm{CP}$ violation due to singlet-doublet mixing. Also one could easily imagine parameter choices in the real NMSSM which could mimic effects of a CP violating NMSSM. Therefore, in order to unambiguously pin down $\mathrm{CP}$ violation, in addition to a combined fit to as many Higgs observables as possible, in particular observables sensitive to $\mathrm{CP}$ violation have to be investigated such as e.g. the angular distributions in Higgs boson decays into $Z$ bosons. In order to correctly interpret the results of these studies, however, the precise predictions of the Higgs boson masses and couplings are indispensible, also including two-loop corrections, the calculation of which is deferred to future work. 


\section{$5 \quad$ Summary and conclusions}

We have calculated the one-loop corrections to the neutral Higgs bosons in the CP-violating NMSSM by applying a mixed renormalization scheme where part of the parameters are renormalized on-shell while $\tan \beta, v_{s}, \lambda, \kappa, A_{\kappa}$ and the CP-violating phases are renormalized in the $\overline{\mathrm{DR}}$ scheme. We have in general allowed for tree-level $\mathrm{CP}$ violation due to non-vanishing phases $\varphi_{u}, \varphi_{s}, \varphi_{\kappa}$ and $\varphi_{\lambda}$, and for loop induced CP violation from the stop sector due to a non-zero phase $\varphi_{A_{t}}$. Several scenarios have been investigated which start from parameter sets that are compatible with the experimental Higgs searches in the limit of the real NMSSM, subsequently CP violation is turned on. As expected the dependence of the one-loop corrected Higgs masses and mixing matrix elements on the CP-violating phase turned out to be more pronounced for tree-level CP violation than for radiatively induced CP violation. The loop corrections were found to considerably change the masses and mixing angles with crucial implications for the Higgs phenomenology at the LHC. As it is well known in the MSSM and the real NMSSM we also found that a scenario may be excluded at tree-level, whereas it is compatible with LHC searches at one-loop. Of special interest is the dependence on the $\mathrm{CP}$-violating phase. It may be rather smooth at tree-level but more pronounced at one-loop so that at one-loop the CP-violating phase under investigation may be much more restricted than at tree-level due to possible non-compatibility with the experiments. Therefore, in order to correctly define viable scenarios and pin down allowed parameter ranges, the inclusion of higher-order corrections is indispensible. This is also true for observables sensitive to $\mathrm{CP}$ violation which have to be investigated in order to unambiguously pin down $\mathrm{CP}$ violation and distinguish $\mathrm{CP}$ violating NMSSM scenarios from parameter choices which could mimic similar effects as expected from non-zero CP phases. We also investigated the theoretical error due to the unknown higher order corrections by applying an on-shell and a $\overline{\mathrm{DR}}$ renormalization scheme for the top and bottom quark mass and by varying the renormalization scale between half and twice its value. The theoretical error of the one-loop corrected Higgs masses can be conservatively estimated to be about $10 \%$.

Note added in Proof. Right after the publication of this work the LHC experiments ATLAS and CMS reported the exciting discovery of a new boson [196] compatible with a SM-like Higgs boson, with a mass of $126.5 \mathrm{GeV}$ at $5 \sigma$ local significance [197] and of $125.3 \mathrm{GeV}$ at $4.9 \sigma$ local significance [197], respectively. The results of our paper, and in particular our criteria for a scenario not being excluded by the LHC searches, remain valid in view of these new results, so that our conclusions are unchanged.

\section{Acknowledgments}

We would like to thank J. Baglio, M. Maniatis, M. Spira and D. Stöckinger for helpful discussions. This research was supported in part by the Deutsche Forschungsgemeinschaft via the Sonderforschungsbereich/Transregio SFB/TR-9 Computational Particle Physics. R.G. acknowledges financial support from the Landesgraduiertenkolleg. 


\section{A Relations between original and physical parameters}

For the transformation of the Lagrangian from the original parameters to the physical ones the following relations are used:

$$
\begin{aligned}
& m_{H_{d}}^{2}=\frac{e}{2 c_{\beta} M_{W} s_{\theta_{W}}} t_{h_{d}}-\left[\frac{M_{Z}^{2} c_{2 \beta}}{2}-v_{s} t_{\beta}|\lambda|\left(\frac{\left|A_{\lambda}\right|}{\sqrt{2}} c_{\varphi_{x}}+|\kappa| \frac{v_{s}}{2} c_{\varphi_{y}}\right)+|\lambda|^{2}\left(\frac{2 s_{\beta}^{2} M_{W}^{2} s_{\theta_{W}}^{2}}{e^{2}}+\frac{v_{s}^{2}}{2}\right)\right], \\
& m_{H_{u}}^{2}=\frac{e}{2 s_{\beta} M_{W} s_{\theta_{W}}} t_{h_{u}}+\left[\frac{M_{Z}^{2} c_{2 \beta}}{2}+\frac{|\lambda| v_{s}}{t_{\beta}}\left(\frac{\left|A_{\lambda}\right|}{\sqrt{2}} c_{\varphi_{x}}+|\kappa| \frac{v_{s}}{2} c_{\varphi_{y}}\right)-|\lambda|^{2}\left(\frac{2 c_{\beta}^{2} M_{W}^{2} s_{\theta_{W}}^{2}}{e^{2}}+\frac{v_{s}^{2}}{2}\right)\right] \text {, } \\
& m_{S}^{2}=\frac{t_{h_{s}}}{v_{s}}+\left[s_{2 \beta}|\lambda|\left(\frac{\left|A_{\lambda}\right|}{\sqrt{2}} c_{\varphi_{x}}+|\kappa| v_{s} c_{\varphi_{y}}\right)-|\lambda|^{2} v_{s}\right] \frac{2 M_{W}^{2} s_{\theta_{W}}^{2}}{e^{2} v_{s}}-|\kappa|^{2} v_{s}^{2}-\frac{1}{\sqrt{2}}\left|A_{\kappa}\right||\kappa| v_{s} c_{\varphi_{z}}, \\
& \varphi_{A_{\lambda}}=\operatorname{sign}_{x}\left[n_{x}(\pi)+(-1)^{n_{x}}\left|\arcsin \left(\frac{e}{\sqrt{2}\left|A_{\lambda}\right| M_{W} s_{\theta_{W}} s_{\beta}|\lambda| v_{s}} t_{a_{d}}+\frac{|\kappa| v_{s}}{\sqrt{2}\left|A_{\lambda}\right|} s_{\varphi_{y}}\right)\right|\right] \\
& -\varphi_{\lambda}-\varphi_{s}-\varphi_{u}, \\
& \varphi_{A_{\kappa}}=\operatorname{sign}_{z}\left[n_{z}(\pi)+(-1)^{n_{z}}\right. \\
& \left.\left|\arcsin \left(\frac{\sqrt{2}}{\left|A_{\kappa}\right| v_{s}}\left[\frac{2 M_{W} s_{\theta_{W}} c_{\beta}}{e|\kappa| v_{s}^{2}} t_{a_{d}}+\frac{3 M_{W}^{2} s_{\theta_{W}}^{2} s_{2 \beta}|\lambda|}{e^{2}} s_{\varphi_{y}}-\frac{1}{|\kappa| v_{s}} t_{a_{s}}\right]\right)\right|\right]-\varphi_{\kappa}-3 \varphi_{s}, \\
& \left|A_{\lambda}\right|=\frac{s_{2 \beta}}{\sqrt{2} c_{\varphi_{x}}|\lambda| v_{s} c_{\Delta \beta}^{2}}\left[M_{H^{ \pm}}^{2}-M_{W}^{2} c_{\Delta \beta}^{2}-|\kappa||\lambda| v_{s}^{2} \frac{c_{\Delta \beta}^{2}}{s_{2 \beta}} c_{\varphi_{y}}+\frac{2 M_{W}^{2} s_{\theta_{W}}^{2} c_{\Delta \beta}^{2}}{e^{2}}|\lambda|^{2}\right. \\
& \left.-\frac{e}{2 M_{W} s_{\theta_{W}}}\left[t_{h_{u}} \frac{c_{\beta_{c}}^{2}}{s_{\beta}}+t_{h_{d}} \frac{s_{\beta_{c}}^{2}}{c_{\beta}}\right]\right] \text {, } \\
& v_{u}=\frac{2 M_{W} s_{\theta_{W}} s_{\beta}}{e}, \quad v_{d}=\frac{2 M_{W} s_{\theta_{W}} c_{\beta}}{e}, \\
& g=\frac{e}{s_{\theta_{W}}}, \quad g^{\prime}=\frac{e}{c_{\theta_{W}}} \quad \text { with } \quad c_{\theta_{W}}=\frac{M_{W}}{M_{Z}} \quad \text { and } \quad s_{\theta_{W}}^{2}=1-c_{\theta_{W}}^{2},
\end{aligned}
$$

where $n_{x}$ and $n_{z}$ can be zero or one in case of two solutions of the tadpole condition, eqs. (3.5) and (3.7), and zero if there exists only a single one. Here, $\operatorname{sign}_{x}$ and $\operatorname{sign}_{z}$ are the sign of the corresponding arcsine evaluated in the interval $[-\pi, \pi)$, respectively.

\section{B Higgs boson mass matrix}

In this section we list the Higgs boson mass matrix elements in a form needed as starting point for the renormalization procedure in the basis $\Phi=\left(h_{d}, h_{u}, h_{s}, A, a_{s}, G\right)^{T}$. This basis 
is obtained by transforming the original basis $\phi=\left(h_{d}, h_{u}, h_{s}, a_{u}, a_{d}, a_{s}\right)^{T}$ with the matrix

$$
\mathcal{R}^{G}=\left(\begin{array}{cc}
1 & 0 \\
0 & \mathcal{U}^{G}
\end{array}\right) \quad \text { and } \quad \mathcal{U}^{G}=\left(\begin{array}{ccc}
s_{\beta_{n}} & c_{\beta_{n}} & 0 \\
0 & 0 & 1 \\
c_{\beta_{n}} & -s_{\beta_{n}} & 0
\end{array}\right) \text {. }
$$

The angle $\beta_{n}$ is chosen such that the Goldstone boson field (with zero mass eigenvalue) is extracted and, at tree-level, coincides with the angle $\beta$ defined via the ratio of the vacuum expectation values, $\beta_{n}=\beta$.

The mass matrix elements of the CP-even part $M_{h h}$, cf. eq. (3.11), are

$$
\begin{aligned}
M_{h_{d} h_{d}}= & {\left[\frac{M_{H^{ \pm}}^{2}}{c_{\Delta \beta}^{2}}-M_{W}^{2}\right] s_{\beta}^{2}+M_{Z}^{2} c_{\beta}^{2}+\frac{e c_{\beta} c_{\beta_{B}}^{2}}{2 M_{W} s_{\theta_{W}} c_{\Delta \beta}^{2}}\left[\left(1+2 t_{\beta} t_{\beta_{B}}\right) t_{h_{d}}-t_{\beta} t_{h_{u}}\right] } \\
& +2|\lambda|^{2} M_{W}^{2} \frac{s_{\theta_{W}}^{2}}{e^{2}} s_{\beta}^{2}, \\
M_{h_{d} h_{u}}= & -\left[\frac{M_{H^{ \pm}}^{2}}{c_{\Delta \beta}^{2}}-M_{W}^{2}+M_{Z}^{2}\right] s_{\beta} c_{\beta}+\frac{e c_{\beta} c_{\beta_{B}}^{2}}{2 M_{W} s_{\theta_{W}} c_{\Delta \beta}^{2}}\left[t_{h_{u}}+t_{\beta} t_{\beta_{B}}^{2} t_{h_{d}}\right] \\
& +|\lambda|^{2} M_{W}^{2} \frac{s_{\theta_{W}}^{2}}{e^{2}} s_{2 \beta}, \\
M_{h_{u} h_{u}}= & {\left[\frac{M_{H^{ \pm}}^{2}}{c_{\Delta \beta}^{2}}-M_{W}^{2}\right] c_{\beta}^{2}+M_{Z}^{2} s_{\beta}^{2}+\frac{e c_{\beta} s_{2 \beta_{B}}}{4 M_{W} s_{\theta_{W}} c_{\Delta \beta}^{2}}\left[\left(2+t_{\beta} t_{\beta_{B}}\right) t_{h_{u}}-t_{\beta_{B}} t_{h_{d}}\right] } \\
& +2|\lambda|^{2} M_{W}^{2} \frac{s_{\theta_{W}}^{2}}{e^{2}} c_{\beta}^{2}, \\
M_{h_{d} h_{s}}= & -\left[\frac{M_{H^{ \pm}}^{2}}{c_{\Delta \beta}^{2}}-M_{W}^{2}\right] \frac{M_{W} s_{\theta_{W}} s_{\beta} s_{2 \beta}}{e v_{s}}+\frac{s_{\beta} c_{\beta} c_{\beta_{B}}^{2}}{v_{s} c_{\Delta \beta}^{2}}\left[t_{h_{u}}+t_{\beta} t_{\beta_{B}}^{2} t_{h_{d}}\right] \\
& +|\lambda| M_{W} \frac{s_{\theta_{W}}}{e} v_{s}\left[2|\lambda| c_{\beta}-|\kappa| s_{\beta} c_{\varphi_{y}}\right]-\frac{4|\lambda|^{2} M_{W}^{3} s_{\theta_{W}}^{3} s_{\beta}^{2} c_{\beta}}{e^{3} v_{s}} \\
& +|\lambda| M_{W}^{2} \frac{s_{\theta_{W}}^{2} s_{2 \beta}}{e^{2} v_{s}^{2}}\left[2|\lambda| M_{W}^{2} \frac{s_{\theta_{W}}^{2}}{e^{2}} s_{2 \beta}-|\kappa| v_{s}^{2} c_{\varphi_{y}}\right]+2|\kappa|^{2} v_{s}^{2}+\frac{1}{\sqrt{2}}\left|A_{\kappa}\right||\kappa| v_{s} c_{\varphi_{z}}, \\
M_{h_{u} h_{s}}= & -\left[\frac{M_{H^{ \pm}}^{2}}{c_{\Delta \beta}^{2}}-M_{W}^{2}\right] \frac{M_{W} s_{\theta_{W}} c_{\beta} s_{2 \beta}}{e v_{s}}+\frac{c_{\beta}^{2} c_{\beta_{B}}^{2}}{v_{s} c_{\Delta \beta}^{2}}\left[t_{h_{u}}+t_{\beta} t_{\beta_{B}}^{2} t_{h_{d}}\right] \\
& +|\lambda| M_{W} \frac{s_{\theta_{W}}}{e} v_{s}\left[2|\lambda| s_{\beta}-|\kappa| c_{\beta} c_{\varphi_{y}}\right]-\frac{4|\lambda|^{2} M_{W}^{3} s_{\theta_{W}}^{3} s_{\beta} c_{\beta}^{2}}{e^{3} v_{s}} \\
M_{h_{s} h_{s}}= & {\left.\left[\frac{M_{H^{ \pm}}^{2}}{c_{\Delta \beta}^{2}}-M_{W}^{2}\right] \frac{M_{W}^{2} s_{\theta_{W}}^{2} s_{2 \beta}^{2}}{e^{2} v_{s}^{2}}-\frac{M_{W} s_{\theta_{W}} s_{2 \beta} c_{\beta} c_{\beta_{B}}^{2}}{t_{h_{u}}}+t_{\beta} t_{\beta_{B}}^{2} t_{h_{d}}\right]+\frac{t_{h_{s}}}{v_{s}} } \\
& (\mathrm{~B} .
\end{aligned}
$$

where $\Delta \beta=\beta-\beta_{B}$ and $\beta_{B} \equiv \beta_{c}=\beta_{n}$. The mixing angle of the charged Higgs bosons, $\beta_{c}$, and the mixing angle $\beta_{n}$, needed for extracting the Goldstone boson, coincide and no discrimination between these two mixing angles is done in the formulae. The angles $\varphi_{x}$, $\varphi_{y}$ and $\varphi_{z}$ have been defined in eqs. (3.8)-(3.10). 
The mass matrix elements corresponding to the CP-odd components of the Higgs boson mass matrix are given as

$$
\begin{aligned}
M_{A A}= & M_{H^{ \pm}}^{2}-M_{W}^{2} c_{\Delta \beta}^{2}+2|\lambda|^{2} M_{W}^{2} \frac{s_{\theta_{W}}^{2}}{e^{2}} c_{\Delta \beta}^{2} \\
M_{A a_{s}}= & {\left[M_{H^{ \pm}}^{2}-M_{W}^{2} c_{\Delta \beta}^{2}\right] \frac{M_{W} s_{\theta_{W}} s_{2 \beta}}{e v_{s} c_{\Delta \beta}}-\frac{c_{\beta} c_{\beta_{B}}^{2}}{v_{s} c_{\Delta \beta}}\left[t_{h_{u}}+t_{\beta} t_{\beta_{B}}^{2} t_{h_{d}}\right] } \\
& +|\lambda| M_{W} \frac{s_{\theta_{W}} c_{\Delta \beta}}{e v_{s}}\left[2|\lambda| M_{W}^{2} \frac{s_{\theta_{W}}^{2}}{e^{2}} s_{2 \beta}-3|\kappa| v_{s}^{2} c_{\varphi_{y}}\right] \\
M_{a_{s} a_{s}}= & {\left[M_{H^{ \pm}}^{2}-M_{W}^{2} c_{\Delta \beta}^{2}\right] \frac{M_{W}^{2} s_{\theta_{W}}^{2} s_{2 \beta}^{2}}{e^{2} v_{s}^{2} c_{\Delta \beta}^{2}}-\frac{M_{W} s_{\theta_{W}} s_{2 \beta} c_{\beta} c_{\beta_{B}}^{2}}{e v_{s}^{2} c_{\Delta \beta}^{2}}\left[t_{h_{u}}+t_{\beta} t_{\beta_{B}}^{2} t_{h_{d}}\right]+\frac{t_{h_{s}}}{v_{s}} } \\
& +|\lambda| M_{W}^{2} \frac{s_{\theta_{W}}^{2} s_{2 \beta}}{e^{2} v_{s}^{2}}\left[2|\lambda| M_{W}^{2} \frac{s_{\theta_{W}}^{2}}{e^{2}} s_{2 \beta}+3|\kappa| v_{s}^{2} c_{\varphi_{y}}\right]-\frac{3}{\sqrt{2}}\left|A_{\kappa}\right||\kappa| v_{s} c_{\varphi_{z}} \\
M_{A G}= & {\left[M_{H^{ \pm}}^{2}-M_{W}^{2} c_{\Delta \beta}^{2}\right] t_{\Delta \beta}+\frac{e c_{\beta_{B}}}{2 M_{W} s_{\theta_{W}} c_{\Delta \beta}}\left[t_{\beta_{B}} t_{h_{d}}-t_{h_{u}}\right]+|\lambda|^{2} M_{W}^{2} \frac{s_{\theta_{W}}^{2}}{e^{2}} s_{2 \Delta \beta}, } \\
M_{a_{s} G}= & {\left[M_{H^{ \pm}}^{2}-M_{W}^{2} c_{\Delta \beta}^{2}\right] \frac{M_{W} s_{\theta_{W}} s_{2 \beta} s_{\Delta \beta}}{e v_{s} c_{\Delta \beta}^{2}}-\frac{c_{\beta} c_{\beta_{B}}^{2} s_{\Delta \beta}}{v_{s} c_{\Delta \beta}^{2}}\left[t_{h_{u}}+t_{\beta} t_{\beta_{B}}^{2} t_{h_{d}}\right] } \\
& +|\lambda| M_{W} \frac{s_{\theta_{W}} s_{\Delta \beta}}{e v_{s}}\left[2|\lambda| M_{W}^{2} \frac{s_{\theta_{W}}^{2}}{e^{2}} s_{2 \beta}-3|\kappa| v_{s}^{2} c_{\varphi_{y}}\right] \\
M_{G G}= & {\left[M_{H^{ \pm}}^{2}-M_{W}^{2} c_{\Delta \beta}^{2}\right] t_{\Delta \beta}^{2}+\frac{e c_{\beta-2 \beta_{B}}}{2 M_{W} s_{\theta_{W}} c_{\Delta \beta}^{2}}\left[t_{h_{d}}-t_{\beta-2 \beta_{B}} t_{h_{u}}\right]+2|\lambda|^{2} M_{W}^{2} \frac{s_{\theta_{W}}^{2}}{e^{2}} s_{\Delta \beta}^{2} . }
\end{aligned}
$$

Finally, the mass matrix elements describing the mixing between the CP-even and the CP-odd components can be expressed as

$$
M_{h a} \mathcal{U}^{\mathcal{G}^{T}}=\left(\begin{array}{ccc}
\frac{e c_{\beta_{B}}}{2 M_{W} s_{\theta_{W}} s_{\beta}} t_{a_{d}} & \frac{1}{v_{s}} t_{a_{d}}+3|\kappa||\lambda| M_{W} \frac{s_{\theta_{W}}}{e} v_{s} s_{\beta} s_{\varphi_{y}} & -\frac{e s_{\beta_{B}}}{2 M_{W} s_{\theta_{W}} s_{\beta}} t_{a_{d}} \\
\frac{e s_{\beta_{B}}}{2 M_{W} s_{\theta_{W}} s_{\beta}} t_{a_{d}} & \frac{1}{v_{s} t_{\beta}} t_{a_{d}}+3|\kappa||\lambda| M_{W} \frac{{ }_{\theta_{W}}}{e} v_{s} c_{\beta} s_{\varphi_{y}} & \frac{e c_{\beta_{B}}}{2 M_{W} s_{B} s_{\beta}} t_{a_{d}} \\
M_{h_{s} A} & M_{h_{s} a_{s}} & M_{h_{s} G}
\end{array}\right)
$$

with

$$
\begin{aligned}
M_{h_{s} A} & =\frac{c_{\Delta \beta}}{v_{s} s_{\beta}} t_{a_{d}}-|\kappa||\lambda| M_{W} \frac{s_{\theta_{W}}}{e} v_{s} c_{\Delta \beta} s_{\varphi_{y}} \\
M_{h_{s} a_{s}} & =\frac{2}{v_{s}} t_{a_{s}}-\frac{4 M_{W} s_{\theta_{W}}}{e}\left[\frac{c_{\beta}}{v_{s}^{2}} t_{a_{d}}+|\kappa||\lambda| M_{W} \frac{s_{\theta_{W}}}{e} s_{2 \beta} s_{\varphi_{y}}\right] \\
M_{h_{s} G} & =\frac{s_{\Delta \beta}}{v_{s} s_{\beta}} t_{a_{d}}-|\kappa||\lambda| M_{W} \frac{s_{\theta_{W}}}{e} v_{s} s_{\Delta \beta} s_{\varphi_{y}} .
\end{aligned}
$$

\section{Chargino and neutralino self-energies}

In this section the expressions for the renormalized self-energies of the charginos and neutralinos are listed. The different parts of the renormalized chargino self-energy decomposed 
according to eq. (3.63) can be written as $(i, j=1,2)$

$$
\begin{aligned}
& {\left[\hat{\Sigma}_{\chi^{+}}^{R}\left(p^{2}\right)\right]_{i j}=\left[\Sigma_{\chi^{+}}^{R}\left(p^{2}\right)\right]_{i j}+\frac{1}{2}\left[U^{*}\left(\delta Z_{R}^{C}+\delta Z_{R}^{C^{*}}\right) U^{T}\right]_{i j},} \\
& {\left[\hat{\Sigma}_{\chi^{+}}^{L}\left(p^{2}\right)\right]_{i j}=\left[\Sigma_{\chi^{+}}^{L}\left(p^{2}\right)\right]_{i j}+\frac{1}{2}\left[V\left(\delta Z_{L}^{C}+\delta Z_{L}^{C^{*}}\right) V^{\dagger}\right]_{i j},} \\
& {\left[\hat{\Sigma}_{\chi^{+}}^{L s}\left(p^{2}\right)\right]_{i j}=\left[\Sigma_{\chi^{+}}^{L s}\left(p^{2}\right)\right]_{i j}-\frac{1}{2} m_{\chi_{k}^{ \pm}}\left(\left[U^{*} \delta Z_{R}^{C} U^{T}\right]_{i k} \delta_{k j}+\delta_{i k}\left[V \delta Z_{L}^{C} V^{\dagger}\right]_{k j}\right)-\left[U^{*} \delta M_{C} V^{\dagger}\right]_{i j},} \\
& {\left[\hat{\Sigma}_{\chi^{+}}^{R s}\left(p^{2}\right)\right]_{i j}=\left[\Sigma_{\chi^{+}}^{R s}\left(p^{2}\right)\right]_{i j}-\frac{1}{2} m_{\chi_{k}^{ \pm}}\left(\left[V \delta Z_{L}^{C^{*}} V^{\dagger}\right]_{i k} \delta_{k j}+\delta_{i k}\left[U^{*} \delta Z_{R}^{C *} U^{T}\right]_{k j}\right)-\left[V \delta M_{C}^{\dagger} U^{T}\right]_{i j},}
\end{aligned}
$$

where, for the renormalization procedure, the chargino spinors as given in eq. (2.8) and the $2 \times 2$ chargino mass matrix $M_{C}$ are replaced by

$$
\begin{aligned}
& \psi_{L}^{+} \rightarrow\left(1+\frac{1}{2} \delta Z_{L}^{C}\right) \psi_{L}^{+}, \\
& \psi_{R}^{-} \rightarrow\left(1+\frac{1}{2} \delta Z_{R}^{C}\right) \psi_{R}^{-}, \quad \text { with } \quad \delta Z_{X}^{C}=\left(\begin{array}{cc}
\delta Z_{X_{1}}^{C} & 0 \\
0 & \delta Z_{X_{2}}^{C}
\end{array}\right) \quad \text { and } \quad X=L, R,
\end{aligned}
$$

and

$$
M_{C} \rightarrow M_{C}+\delta M_{C},
$$

respectively, where $\delta M_{C}$ is given in eq. (3.68).

The various parts of the decomposed renormalized neutralino self-energy can be expressed as $(i, j=1, \ldots, 5)$

$$
\begin{aligned}
{\left[\hat{\Sigma}_{\chi^{0}}^{R}\left(p^{2}\right)\right]_{i j}=} & {\left[\Sigma_{\chi^{0}}^{R}\left(p^{2}\right)\right]_{i j}+\frac{1}{2}\left[\mathcal{N}^{*}\left(\delta Z^{N}+\delta Z^{N^{*}}\right) \mathcal{N}^{T}\right]_{i j}, } \\
{\left[\hat{\Sigma}_{\chi^{0}}^{L}\left(p^{2}\right)\right]_{i j}=} & {\left[\Sigma_{\chi^{0}}^{L}\left(p^{2}\right)\right]_{i j}+\frac{1}{2}\left[\mathcal{N}\left(\delta Z^{N}+\delta Z^{N^{*}}\right) \mathcal{N}^{\dagger}\right]_{i j}, } \\
{\left[\hat{\Sigma}_{\chi^{0}}^{L s}\left(p^{2}\right)\right]_{i j}=} & {\left[\Sigma_{\chi^{0}}^{L s}\left(p^{2}\right)\right]_{i j}-\frac{1}{2} m_{\chi_{k}^{0}}\left(\left[\mathcal{N}^{*} \delta Z^{N} \mathcal{N}^{\dagger}\right]_{i k} \delta_{k j}+\delta_{i k}\left[\mathcal{N}^{*} \delta Z^{N} \mathcal{N}^{\dagger}\right]_{k j}\right) } \\
& -\left[\mathcal{N}^{*} \delta M_{N} \mathcal{N}^{\dagger}\right]_{i j}, \\
{\left[\hat{\Sigma}_{\chi^{0}}^{R s}\left(p^{2}\right)\right]_{i j}=} & {\left[\Sigma_{\chi^{0}}^{R s}\left(p^{2}\right)\right]_{i j}-\frac{1}{2} m_{\chi_{k}^{0}}\left(\left[\mathcal{N} \delta Z^{N^{*}} \mathcal{N}^{T}\right]_{i k} \delta_{j k}+\delta_{i k}\left[\mathcal{N} \delta Z^{N^{*}} \mathcal{N}^{T}\right]_{k j}\right) } \\
& -\left[\mathcal{N} \delta M_{N}^{\dagger} \mathcal{N}^{T}\right]_{i j} .
\end{aligned}
$$

For the renormalization procedure, the neutralino spinor defined in eq. (2.7) has been replaced by

$$
\psi^{0} \rightarrow\left(1+\frac{1}{2} \delta Z^{N}\right) \psi^{0} \quad \text { with } \quad \delta Z^{N}=\operatorname{diag}\left(\delta Z_{1}^{N}, \delta Z_{2}^{N}, \delta Z_{3}^{N}, \delta Z_{4}^{N}, \delta Z_{5}^{N}\right)
$$

and the neutralino mass matrix by

$$
M_{N} \rightarrow M_{N}+\delta M_{N}
$$

The matrix elements of $\delta M_{N}$ can be found in eqs. (3.76)-(3.86). 
Open Access. This article is distributed under the terms of the Creative Commons Attribution License which permits any use, distribution and reproduction in any medium, provided the original author(s) and source are credited.

\section{References}

[1] CMS collaboration, S. Chatrchyan et al., Search for a Higgs boson in the decay channel $H \rightarrow Z Z^{(*)} \rightarrow q \bar{q} l^{-} l^{+}$in pp collisions at $\sqrt{s}=7 \mathrm{TeV}$, JHEP 04 (2012) 036 [arXiv: 1202.1416] [INSPIRE].

[2] CMS collaboration, S. Chatrchyan et al., Search for the standard model Higgs boson decaying into two photons in pp collisions at $\sqrt{s}=7$ TeV, Phys. Lett. B 710 (2012) 403 [arXiv:1202.1487] [INSPIRE].

[3] CMS collaboration, S. Chatrchyan et al., Combined results of searches for the standard model Higgs boson in pp collisions at $\sqrt{s}=7$ TeV, Phys. Lett. B 710 (2012) 26 [arXiv: 1202.1488] [INSPIRE].

[4] CMS collaboration, S. Chatrchyan et al., Search for the standard model Higgs boson decaying to a $W$ pair in the fully leptonic final state in pp collisions at $\sqrt{s}=7 \mathrm{TeV}$, Phys. Lett. B $\mathbf{7 1 0}$ (2012) 91 [arXiv: 1202.1489] [INSPIRE].

[5] CMS collaboration, S. Chatrchyan et al., Search for the standard model Higgs boson in the decay channel $H \rightarrow Z Z \rightarrow 4$ leptons in pp collisions at $\sqrt{s}=7 \mathrm{TeV}$, Phys. Rev. Lett. 108 (2012) 111804 [arXiv:1202.1997] [InSPIRE].

[6] CMS collaboration, S. Chatrchyan et al., Search for the standard model Higgs boson in the $H \rightarrow Z Z \rightarrow 2 \ell 2 \nu$ channel in pp collisions at $\sqrt{s}=7$ TeV, JHEP 03 (2012) 040 [arXiv: 1202.3478] [INSPIRE].

[7] CMS collaboration, S. Chatrchyan et al., Search for the standard model Higgs boson in the $H \rightarrow Z Z \rightarrow \ell^{+} \ell^{-} \tau^{+} \tau^{-}$decay channel in pp collisions at $\sqrt{s}=7 \mathrm{TeV}$, JHEP 03 (2012) 081 [arXiv:1202.3617] [INSPIRE].

[8] CMS collaboration, S. Chatrchyan et al., Search for neutral Higgs bosons decaying to tau pairs in pp collisions at $\sqrt{s}=7$ TeV, Phys. Lett. B 713 (2012) 68 [arXiv:1202.4083] [INSPIRE].

[9] CMS collaboration, S. Chatrchyan et al., Search for the standard model Higgs boson decaying to bottom quarks in pp collisions at $\sqrt{s}=7$ TeV, Phys. Lett. B 710 (2012) 284 [arXiv: 1202.4195] [INSPIRE].

[10] ATLAS collaboration, G. Aad et al., Combined search for the Standard Model Higgs boson using up to $4.9 \mathrm{fb}^{-1}$ of pp collision data at $\sqrt{s}=7 \mathrm{TeV}$ with the ATLAS detector at the LHC, Phys. Lett. B $\mathbf{7 1 0 ~ ( 2 0 1 2 )} 49$ [arXiv:1202.1408] [INSPIRE].

[11] ATLAS collaboration, G. Aad et al., Search for the Standard Model Higgs boson in the diphoton decay channel with $4.9 \mathrm{fb}^{-1}$ of pp collisions at $\sqrt{s}=7 \mathrm{TeV}$ with ATLAS, Phys. Rev. Lett. 108 (2012) 111803 [arXiv:1202.1414] [INSPIRE].

[12] ATLAS collaboration, G. Aad et al., Search for the Standard Model Higgs boson in the decay channel $H \rightarrow Z Z^{(*)} \rightarrow 4 \ell$ with $4.8 \mathrm{fb}^{-1}$ of pp collision data at $\sqrt{s}=7 \mathrm{TeV}$ with ATLAS, Phys. Lett. B 710 (2012) 383 [arXiv:1202.1415] [InSPIRE].

[13] ATLAS collaboration, G. Aad et al., Search for the Standard Model Higgs boson in the $H \rightarrow W W^{(*)} \rightarrow \ell \nu \ell \nu$ decay mode with $4.7 / f b$ of ATLAS data at $\sqrt{s}=7$ TeV, Phys. Lett. B 716 (2012) 62 [arXiv:1206.0756] [INSPIRE]. 
[14] ATLAS collaboration, G. Aad et al., Search for a Standard Model Higgs boson in the mass range 200-600 GeV in the $H \rightarrow Z Z \rightarrow \ell^{+} \ell^{-} q \bar{q}$ decay channel, arXiv:1206.2443 [INSPIRE].

[15] ATLAS collaboration, G. Aad et al., Search for the Standard Model Higgs boson in the $H \rightarrow \tau^{+} \tau^{-}$decay mode in $\sqrt{s}=7$ TeV pp collisions with ATLAS, JHEP 09 (2012) 070 [arXiv: 1206.5971] [INSPIRE].

[16] ATLAS collaboration, G. Aad et al., Search for the Higgs boson in the $H \rightarrow W W \rightarrow \ell \nu j j$ decay channel at $\sqrt{s}=7$ TeV with the ATLAS detector, arXiv:1206.6074 [INSPIRE].

[17] ATLAS collaboration, G. Aad et al., Search for a Standard Model Higgs boson in the $H \rightarrow Z Z \rightarrow l^{+} l^{-} \nu \bar{\nu}$ decay channel using $4.7 \mathrm{fb}^{-1}$ of $\sqrt{s}=7$ TeV data with the ATLAS detector, arXiv:1205.6744 [INSPIRE].

[18] D. Volkov and V. Akulov, Is the Neutrino a Goldstone Particle?, Phys. Lett. B 46 (1973) 109 [INSPIRE].

[19] J. Wess and B. Zumino, Supergauge Transformations in Four-Dimensions, Nucl. Phys. B 70 (1974) 39 [INSPIRE].

[20] P. Fayet, Supersymmetry and Weak, Electromagnetic and Strong Interactions, Phys. Lett. B 64 (1976) 159 [INSPIRE].

[21] P. Fayet, Spontaneously Broken Supersymmetric Theories of Weak, Electromagnetic and Strong Interactions, Phys. Lett. B 69 (1977) 489 [InSPIRE].

[22] P. Fayet, Relations Between the Masses of the Superpartners of Leptons and Quarks, the Goldstino Couplings and the Neutral Currents, Phys. Lett. B 84 (1979) 416 [INSPIRE].

[23] G.R. Farrar and P. Fayet, Phenomenology of the Production, Decay and Detection of New Hadronic States Associated with Supersymmetry, Phys. Lett. B 76 (1978) 575 [InSPIRE].

[24] S. Dimopoulos and H. Georgi, Softly Broken Supersymmetry and SU(5), Nucl. Phys. B 193 (1981) 150 [INSPIRE].

[25] N. Sakai, Naturalness in Supersymmetric Guts, Z. Phys. C 11 (1981) 153 [INSPIRE].

[26] E. Witten, Dynamical Breaking of Supersymmetry, Nucl. Phys. B 188 (1981) 513 [InSPIRE].

[27] H.P. Nilles, Supersymmetry, Supergravity and Particle Physics, Phys. Rept. 110 (1984) 1 [INSPIRE].

[28] H.E. Haber and G.L. Kane, The Search for Supersymmetry: Probing Physics Beyond the Standard Model, Phys. Rept. 117 (1985) 75 [INSPIRE].

[29] M. Sohnius, Introducing Supersymmetry, Phys. Rept. 128 (1985) 39 [InSPIRE].

[30] J. Gunion and H.E. Haber, Higgs Bosons in Supersymmetric Models. 1., Nucl. Phys. B 272 (1986) 1 [Erratum ibid. B 402 (1993) 567-569] [INSPIRE].

[31] J. Gunion and H.E. Haber, Higgs Bosons in Supersymmetric Models. 2. Implications for Phenomenology, Nucl. Phys. B 278 (1986) 449 [INSPIRE].

[32] A. Lahanas and D.V. Nanopoulos, The Road to No Scale Supergravity, Phys. Rept. 145 (1987) 1 [INSPIRE].

[33] J.F. Gunion, H.E. Haber, G. Kane and S. Dawson, The Higgs Hunter's Guide, Addison-Wesley, (1990).

[34] S.P. Martin, A Supersymmetry primer, hep-ph/9709356 [INSPIRE].

[35] S. Dawson, The MSSM and why it works, hep-ph/9712464 [INSPIRE]. 
[36] M. Gomez-Bock et al., Rompimiento de la simetria electrodebil y la fisica del Higgs: Conceptos basicos, J. Phys. Conf. Ser. 18 (2005) 74 [hep-ph/0509077] [InSPIRE].

[37] M. Gomez-Bock, M. Mondragon, M. Muhlleitner, M. Spira and P. Zerwas, Concepts of Electroweak Symmetry Breaking and Higgs Physics, arXiv:0712.2419 [INSPIRE].

[38] A. Djouadi, The Anatomy of electro-weak symmetry breaking. II. The Higgs bosons in the minimal supersymmetric model, Phys. Rept. 459 (2008) 1 [hep-ph/0503173] [INSPIRE].

[39] P. Fayet, Supergauge Invariant Extension of the Higgs Mechanism and a Model for the electron and Its Neutrino, Nucl. Phys. B 90 (1975) 104 [INSPIRE].

[40] R. Barbieri, S. Ferrara and C.A. Savoy, Gauge Models with Spontaneously Broken Local Supersymmetry, Phys. Lett. B 119 (1982) 343 [INSPIRE].

[41] M. Dine, W. Fischler and M. Srednicki, A Simple Solution to the Strong CP Problem with a Harmless Axion, Phys. Lett. B 104 (1981) 199 [InSPIRE].

[42] H.P. Nilles, M. Srednicki and D. Wyler, Weak Interaction Breakdown Induced by Supergravity, Phys. Lett. B 120 (1983) 346 [INSPIRE].

[43] J. Frere, D. Jones and S. Raby, Fermion Masses and Induction of the Weak Scale by Supergravity, Nucl. Phys. B 222 (1983) 11 [INSPIRE].

[44] J. Derendinger and C.A. Savoy, Quantum Effects and $\mathrm{SU}(2) \times \mathrm{U}(1)$ Breaking in Supergravity Gauge Theories, Nucl. Phys. B 237 (1984) 307 [INSPIRE].

[45] J.R. Ellis, J. Gunion, H.E. Haber, L. Roszkowski and F. Zwirner, Higgs Bosons in a Nonminimal Supersymmetric Model, Phys. Rev. D 39 (1989) 844 [INSPIRE].

[46] M. Drees, Supersymmetric Models with Extended Higgs Sector, Int. J. Mod. Phys. A 4 (1989) 3635 [INSPIRE].

[47] U. Ellwanger, M. Rausch de Traubenberg and C.A. Savoy, Particle spectrum in supersymmetric models with a gauge singlet, Phys. Lett. B 315 (1993) 331 [hep-ph/9307322] [INSPIRE].

[48] U. Ellwanger, M. Rausch de Traubenberg and C.A. Savoy, Higgs phenomenology of the supersymmetric model with a gauge singlet, Z. Phys. C 67 (1995) 665 [hep-ph/9502206] [INSPIRE].

[49] U. Ellwanger, M. Rausch de Traubenberg and C.A. Savoy, Phenomenology of supersymmetric models with a singlet, Nucl. Phys. B 492 (1997) 21 [hep-ph/9611251] [INSPIRE].

[50] T. Elliott, S. King and P. White, Unification constraints in the next-to-minimal supersymmetric standard model, Phys. Lett. B 351 (1995) 213 [hep-ph/9406303] [INSPIRE].

[51] S. King and P. White, Resolving the constrained minimal and next-to-minimal supersymmetric standard models, Phys. Rev. D 52 (1995) 4183 [hep-ph/9505326] [InSPIRE].

[52] F. Franke and H. Fraas, Neutralinos and Higgs bosons in the next-to-minimal supersymmetric standard model, Int. J. Mod. Phys. A 12 (1997) 479 [hep-ph/9512366] [INSPIRE].

[53] M. Maniatis, The Next-to-Minimal Supersymmetric extension of the Standard Model reviewed, Int. J. Mod. Phys. A 25 (2010) 3505 [arXiv:0906. 0777] [InSPIRE].

[54] U. Ellwanger, C. Hugonie and A.M. Teixeira, The Next-to-Minimal Supersymmetric Standard Model, Phys. Rept. 496 (2010) 1 [arXiv:0910.1785] [INSPIRE]. 
[55] J.E. Kim and H.P. Nilles, The mu Problem and the Strong CP Problem, Phys. Lett. B 138 (1984) 150 [INSPIRE].

[56] M. Bastero-Gil, C. Hugonie, S. King, D. Roy and S. Vempati, Does LEP prefer the NMSSM?, Phys. Lett. B 489 (2000) 359 [hep-ph/0006198] [INSPIRE].

[57] A. Delgado, C. Kolda, J.P. Olson and A. de la Puente, Solving the Little Hierarchy Problem with a Singlet and Explicit $\mu$ Terms, Phys. Rev. Lett. 105 (2010) 091802 [arXiv: 1005.1282] [INSPIRE].

[58] U. Ellwanger, G. Espitalier-Noel and C. Hugonie, Naturalness and Fine Tuning in the NMSSM: Implications of Early LHC Results, JHEP 09 (2011) 105 [arXiv:1107.2472] [INSPIRE].

[59] G.G. Ross and K. Schmidt-Hoberg, The Fine-Tuning of the Generalised NMSSM, Nucl. Phys. B 862 (2012) 710 [arXiv:1108.1284] [INSPIRE].

[60] S. King, M. Muhlleitner and R. Nevzorov, NMSSM Higgs Benchmarks Near 125 GeV, Nucl. Phys. B 860 (2012) 207 [arXiv:1201.2671] [InSPIRE].

[61] L.J. Hall, D. Pinner and J.T. Ruderman, A Natural SUSY Higgs Near 126 GeV, JHEP 04 (2012) 131 [arXiv: 1112.2703] [INSPIRE].

[62] U. Ellwanger, Enhanced di-photon Higgs signal in the Next-to-Minimal Supersymmetric Standard Model, Phys. Lett. B 698 (2011) 293 [arXiv:1012.1201] [INSPIRE].

[63] U. Ellwanger, A Higgs boson near $125 \mathrm{GeV}$ with enhanced di-photon signal in the NMSSM, JHEP 03 (2012) 044 [arXiv:1112.3548] [INSPIRE].

[64] A. Arvanitaki and G. Villadoro, A Non Standard Model Higgs at the LHC as a Sign of Naturalness, JHEP 02 (2012) 144 [arXiv:1112.4835] [INSPIRE].

[65] E. Accomando et al., Workshop on CP Studies and Non-Standard Higgs Physics, hep-ph/0608079 [INSPIRE].

[66] A. Pilaftsis, Higgs scalar - pseudoscalar mixing in the minimal supersymmetric standard model, Phys. Lett. B 435 (1998) 88 [hep-ph/9805373] [INSPIRE].

[67] D.A. Demir, Effects of the supersymmetric phases on the neutral Higgs sector, Phys. Rev. D 60 (1999) 055006 [hep-ph/9901389] [INSPIRE].

[68] A. Pilaftsis and C.E. Wagner, Higgs bosons in the minimal supersymmetric standard model with explicit CP-violation, Nucl. Phys. B 553 (1999) 3 [hep-ph/9902371] [INSPIRE].

[69] S. Choi, M. Drees and J.S. Lee, Loop corrections to the neutral Higgs boson sector of the MSSM with explicit CP-violation, Phys. Lett. B 481 (2000) 57 [hep-ph/0002287] [InSPIRE].

[70] M.S. Carena, J.R. Ellis, A. Pilaftsis and C. Wagner, Renormalization group improved effective potential for the MSSM Higgs sector with explicit CP-violation, Nucl. Phys. B 586 (2000) 92 [hep-ph/0003180] [INSPIRE].

[71] T. Ibrahim and P. Nath, Corrections to the Higgs boson masses and mixings from chargino, $W$ and charged Higgs exchange loops and large CP phases, Phys. Rev. D 63 (2001) 035009 [hep-ph/0008237] [INSPIRE].

[72] T. Ibrahim and P. Nath, Neutralino exchange corrections to the Higgs boson mixings with explicit CP-violation, Phys. Rev. D 66 (2002) 015005 [hep-ph/0204092] [INSPIRE].

[73] S. Heinemeyer, The Higgs boson sector of the complex MSSM in the Feynman diagrammatic approach, Eur. Phys. J. C 22 (2001) 521 [hep-ph/0108059] [INSPIRE]. 
[74] M.S. Carena, J.R. Ellis, A. Pilaftsis and C. Wagner, Higgs boson pole masses in the MSSM with explicit CP-violation, Nucl. Phys. B 625 (2002) 345 [hep-ph/0111245] [InSPIRE].

[75] S. Ham, S. Oh, E. Yoo, C. Kim and D. Son, Neutral Higgs boson masses of the MSSM at the one loop level in an explicit CP-violation scenario, Phys. Rev. D 68 (2003) 055003 [hep-ph/0205244] [INSPIRE].

[76] M. Frank, S. Heinemeyer, W. Hollik and G. Weiglein, The Higgs boson masses of the complex MSSM: A Complete one loop calculation, hep-ph/0212037 [INSPIRE].

[77] S. Heinemeyer, MSSM Higgs physics at higher orders, Int. J. Mod. Phys. A 21 (2006) 2659 [hep-ph/0407244] [INSPIRE].

[78] S. Heinemeyer, W. Hollik, H. Rzehak and G. Weiglein, The Higgs sector of the complex MSSM at two-loop order: QCD contributions, Phys. Lett. B 652 (2007) 300 [arXiv:0705.0746] [INSPIRE].

[79] M. Frank, T. Hahn, S. Heinemeyer, W. Hollik, H. Rzehak and G. Weiglein, The Higgs Boson Masses and Mixings of the Complex MSSM in the Feynman-Diagrammatic Approach, JHEP 02 (2007) 047 [hep-ph/0611326] [INSPIRE].

[80] E. Christova, H. Eberl, W. Majerotto and S. Kraml, CP violation in charged Higgs decays in the MSSM with complex parameters, Nucl. Phys. B 639 (2002) 263 [Erratum ibid. B 647 (2002) 359-360] [hep-ph/0205227] [INSPIRE].

[81] E. Christova, H. Eberl, W. Majerotto and S. Kraml, CP violation in charged Higgs boson decays into tau and neutrino, JHEP 12 (2002) 021 [hep-ph/0211063] [INSPIRE].

[82] W. Khater and P. Osland, CP violation in top quark production at the LHC and two Higgs doublet models, Nucl. Phys. B 661 (2003) 209 [hep-ph/0302004] [InSPIRE].

[83] S. Choi, J. Kalinowski, Y. Liao and P. Zerwas, H/A Higgs mixing in CP-noninvariant supersymmetric theories, Eur. Phys. J. C 40 (2005) 555 [hep-ph/0407347] [INSPIRE].

[84] K. Williams and G. Weiglein, Precise predictions for $h_{a} \rightarrow h_{b} h_{c}$ decays in the complex MSSM, Phys. Lett. B 660 (2008) 217 [arXiv:0710.5320] [INSPIRE].

[85] K.E. Williams, H. Rzehak and G. Weiglein, Higher order corrections to Higgs boson decays in the MSSM with complex parameters, Eur. Phys. J. C 71 (2011) 1669 [arXiv:1103.1335] [INSPIRE].

[86] M.S. Carena, J.R. Ellis, A. Pilaftsis and C. Wagner, CP violating MSSM Higgs bosons in the light of LEP-2, Phys. Lett. B 495 (2000) 155 [hep-ph/0009212] [INSPIRE].

[87] M.S. Carena, J.R. Ellis, S. Mrenna, A. Pilaftsis and C. Wagner, Collider probes of the MSSM Higgs sector with explicit CP-violation, Nucl. Phys. B 659 (2003) 145 [hep-ph/0211467] [INSPIRE].

[88] A. Dedes and S. Moretti, Effect of large supersymmetric phases on Higgs production., Phys. Rev. Lett. 84 (2000) 22 [hep-ph/9908516] [INSPIRE].

[89] A. Dedes and S. Moretti, Effects of CP-violating phases on Higgs boson production at hadron colliders in the minimal supersymmetric standard model, Nucl. Phys. B 576 (2000) 29 [hep-ph/9909418] [INSPIRE].

[90] S. Choi and J.S. Lee, MSSM Higgs boson production at hadron colliders with explicit CP-violation, Phys. Rev. D 61 (2000) 115002 [hep-ph/9910557] [INSPIRE].

[91] S. Choi, K. Hagiwara and J.S. Lee, Observability of the lightest MSSM Higgs boson with explicit CP-violation via gluon fusion at the LHC, Phys. Lett. B 529 (2002) 212 [hep-ph/0110138] [INSPIRE]. 
[92] A. Arhrib, D.K. Ghosh and O.C. Kong, Observing CP-violating MSSM Higgs bosons at hadron colliders?, Phys. Lett. B 537 (2002) 217 [hep-ph/0112039] [INSPIRE].

[93] B. Cox, J.R. Forshaw, J. Lee, J. Monk and A. Pilaftsis, Observing a light CP-violating Higgs boson in diffraction, Phys. Rev. D 68 (2003) 075004 [hep-ph/0303206] [INSPIRE].

[94] A. Akeroyd, Searching for a very light Higgs boson at the Tevatron, Phys. Rev. D 68 (2003) 077701 [hep-ph/0306045] [INSPIRE].

[95] F. Borzumati, J.S. Lee and W.Y. Song, Threshold corrections to $m(b)$ and the $b \bar{b} \rightarrow H 0(i)$ production in CP-violating SUSY scenarios, Phys. Lett. B 595 (2004) 347 [hep-ph/0401024] [INSPIRE].

[96] V. Khoze, A. Martin and M. Ryskin, Hunting a light CP-violating Higgs via diffraction at the LHC, Eur. Phys. J. C 34 (2004) 327 [hep-ph/0401078] [INSPIRE].

[97] J.R. Ellis, J.S. Lee and A. Pilaftsis, CERN LHC signatures of resonant CP-violation in a minimal supersymmetric Higgs sector, Phys. Rev. D 70 (2004) 075010 [hep-ph/0404167] [INSPIRE].

[98] D.K. Ghosh, R. Godbole and D. Roy, Probing the CP-violating light neutral Higgs in the charged Higgs decay at the LHC, Phys. Lett. B 628 (2005) 131 [hep-ph/0412193] [inSPIRE].

[99] D.K. Ghosh and S. Moretti, Probing the light neutral Higgs boson scenario of the CP-violating MSSM Higgs sector at the LHC, Eur. Phys. J. C 42 (2005) 341 [hep-ph/0412365] [INSPIRE].

[100] J.R. Ellis, J.S. Lee and A. Pilaftsis, Diffraction as a CP and lineshape analyzer for MSSM Higgs bosons at the LHC, Phys. Rev. D 71 (2005) 075007 [hep-ph/0502251] [INSPIRE].

[101] J. Romao, Spontaneous CP-violation in SUSY models: a No Go theorem, Phys. Lett. B 173 (1986) 309 [INSPIRE].

[102] B. Regan, E. Commins, C. Schmidt and D. DeMille, New limit on the electron electric dipole moment, Phys. Rev. Lett. 88 (2002) 071805 [InSPIRE].

[103] C. Baker et al., An Improved experimental limit on the electric dipole moment of the neutron, Phys. Rev. Lett. 97 (2006) 131801 [hep-ex/0602020] [INSPIRE].

[104] W. Griffith et al., Improved Limit on the Permanent Electric Dipole Moment of Hg-199, Phys. Rev. Lett. 102 (2009) 101601 [INSPIRE].

[105] M. Matsuda and M. Tanimoto, Explicit CP-violation of the Higgs sector in the next-to-minimal supersymmetric standard model, Phys. Rev. D 52 (1995) 3100 [hep-ph/9504260] [INSPIRE].

[106] N. Haba, Explicit CP-violation in the Higgs sector of the next-to-minimal supersymmetric standard model, Prog. Theor. Phys. 97 (1997) 301 [hep-ph/9608357] [INSPIRE].

[107] T. Ibrahim and P. Nath, The Neutron and the lepton EDMs in MSSM, large CP-violating phases and the cancellation mechanism, Phys. Rev. D 58 (1998) 111301 [Erratum ibid. D 60 (1999) 099902] [hep-ph/9807501] [INSPIRE].

[108] J.R. Ellis, J.S. Lee and A. Pilaftsis, Electric Dipole Moments in the MSSM Reloaded, JHEP 10 (2008) 049 [arXiv: 0808.1819] [INSPIRE].

[109] M. Boz, The Higgs sector and electron electric dipole moment in next-to-minimal supersymmetry with explicit CP-violation, Mod. Phys. Lett. A 21 (2006) 243 [hep-ph/0511072] [INSPIRE].

[110] K. Cheung, T.-J. Hou, J.S. Lee and E. Senaha, Higgs Mediated EDMs in the Next-to-MSSM: An Application to Electroweak Baryogenesis, Phys. Rev. D 84 (2011) 015002 [arXiv: 1102.5679] [INSPIRE]. 
[111] R. Garisto, Moderate supersymmetric CP-violation, Phys. Rev. D 49 (1994) 4820 [hep-ph/9311249] [INSPIRE].

[112] S. Ham, J. Kim, S. Oh and D. Son, The Charged Higgs boson in the next-to-minimal supersymmetric standard model with explicit CP-violation, Phys. Rev. D 64 (2001) 035007 [hep-ph/0104144] [INSPIRE].

[113] S. Ham, S. Kim, S. OH and D. Son, Higgs bosons of the NMSSM with explicit CP-violation at the ILC, Phys. Rev. D 76 (2007) 115013 [arXiv:0708.2755] [INSPIRE].

[114] S. Ham, S. Oh and D. Son, Neutral Higgs sector of the next-to-minimal supersymmetric standard model with explicit CP-violation, Phys. Rev. D 65 (2002) 075004 [hep-ph/0110052] [INSPIRE].

[115] S. Ham, Y. Jeong and S. Oh, Radiative CP-violation in the Higgs sector of the next-to-minimal supersymmetric model, hep-ph/0308264 [INSPIRE].

[116] K. Funakubo and S. Tao, The Higgs sector in the next-to-MSSM, Prog. Theor. Phys. 113 (2005) 821 [hep-ph/0409294] [INSPIRE].

[117] K. Cheung, T.-J. Hou, J.S. Lee and E. Senaha, The Higgs Boson Sector of the Next-to-MSSM with CP-violation, Phys. Rev. D 82 (2010) 075007 [arXiv:1006.1458] [INSPIRE].

[118] U. Ellwanger, Radiative corrections to the neutral Higgs spectrum in supersymmetry with a gauge singlet, Phys. Lett. B 303 (1993) 271 [hep-ph/9302224] [INSPIRE].

[119] T. Elliott, S. King and P. White, Supersymmetric Higgs bosons at the limit, Phys. Lett. B 305 (1993) 71 [hep-ph/9302202] [INSPIRE].

[120] T. Elliott, S. King and P. White, Squark contributions to Higgs boson masses in the next-to-minimal supersymmetric standard model, Phys. Lett. B 314 (1993) 56 [hep-ph/9305282] [INSPIRE].

[121] T. Elliott, S. King and P. White, Radiative corrections to Higgs boson masses in the next-to-minimal supersymmetric Standard Model, Phys. Rev. D 49 (1994) 2435 [hep-ph/9308309] [INSPIRE].

[122] P. Pandita, Radiative corrections to the scalar Higgs masses in a nonminimal supersymmetric Standard Model, Z. Phys. C 59 (1993) 575 [INSPIRE].

[123] P. Pandita, One loop radiative corrections to the lightest Higgs scalar mass in nonminimal supersymmetric Standard Model, Phys. Lett. B 318 (1993) 338 [INSPIRE].

[124] U. Ellwanger and C. Hugonie, Yukawa induced radiative corrections to the lightest Higgs boson mass in the NMSSM, Phys. Lett. B 623 (2005) 93 [hep-ph/0504269] [INSPIRE].

[125] G. Degrassi and P. Slavich, On the radiative corrections to the neutral Higgs boson masses in the NMSSM, Nucl. Phys. B 825 (2010) 119 [arXiv:0907.4682] [INSPIRE].

[126] F. Staub, W. Porod and B. Herrmann, The Electroweak sector of the NMSSM at the one-loop level, JHEP 10 (2010) 040 [arXiv:1007.4049] [INSPIRE].

[127] K. Ender, T. Graf, M. Muhlleitner and H. Rzehak, Analysis of the NMSSM Higgs Boson Masses at One-Loop Level, Phys. Rev. D 85 (2012) 075024 [arXiv:1111.4952] [INSPIRE].

[128] LEP Working Group for Higgs boson searches, ALEPH, DELPHI, L3, OPAL collaborations, R. Barate et al., Search for the standard model Higgs boson at LEP, Phys. Lett. B 565 (2003) 61 [hep-ex/0306033] [INSPIRE].

[129] AlePh, Delphi, L3, OPAL, LEP Working Group for Higgs Boson Searches collaborations, S. Schael et al., Search for neutral MSSM Higgs bosons at LEP, Eur. Phys. J. C 47 (2006) 547 [hep-ex/0602042] [INSPIRE]. 
[130] TevnPh (Tevatron New Phenomina and Higgs Working Group), CDF, D0 collaborations, Combined CDF and DO Search for Standard Model Higgs Boson Production with up to $10.0 \mathrm{fb}^{-1}$ of Data, arXiv:1203.3774 [INSPIRE].

[131] M. Kobayashi and T. Maskawa, CP Violation in the Renormalizable Theory of Weak Interaction, Prog. Theor. Phys. 49 (1973) 652 [InSPIRE].

[132] A. Denner, Techniques for calculation of electroweak radiative corrections at the one loop level and results for W physics at LEP-200, Fortsch. Phys. 41 (1993) 307 [arXiv:0709.1075] [INSPIRE].

[133] A. Brignole, Radiative corrections to the supersymmetric neutral Higgs boson masses, Phys. Lett. B 281 (1992) 284 [INSPIRE].

[134] P.H. Chankowski, S. Pokorski and J. Rosiek, One loop corrections to the supersymmetric Higgs boson couplings and LEP phenomenology, Phys. Lett. B 286 (1992) 307 [INSPIRE].

[135] P.H. Chankowski, S. Pokorski and J. Rosiek, Complete on-shell renormalization scheme for the minimal supersymmetric Higgs sector, Nucl. Phys. B 423 (1994) 437 [hep-ph/9303309] [INSPIRE].

[136] A. Dabelstein, The One loop renormalization of the MSSM Higgs sector and its application to the neutral scalar Higgs masses, Z. Phys. C 67 (1995) 495 [hep-ph/9409375] [INSPIRE].

[137] A. Dabelstein, Fermionic decays of neutral MSSM Higgs bosons at the one loop level, Nucl. Phys. B 456 (1995) 25 [hep-ph/9503443] [INSPIRE].

[138] A. Freitas and D. Stöckinger, Gauge dependence and renormalization of tan beta in the MSSM, Phys. Rev. D 66 (2002) 095014 [hep-ph/0205281] [INSPIRE].

[139] M. Frank, Radiative corrections to the Higgs sector of the minimal supersymmetric standard model with CP violation, Berlin, Germany: RHOMBOS-Verl. (2003).

[140] W. Hollik, E. Kraus, M. Roth, C. Rupp, K. Sibold and D. Stockinger, Renormalization of the minimal supersymmetric standard model, Nucl. Phys. B 639 (2002) 3 [hep-ph/0204350] [INSPIRE].

[141] J. Kublbeck, M. Böhm and A. Denner, Feyn Arts: Computer Algebraic Generation Of Feynman Graphs And Amplitudes, Comput. Phys. Commun. 60 (1990) 165 [INSPIRE].

[142] T. Hahn, Generating Feynman diagrams and amplitudes with FeynArts 3, Comput. Phys. Commun. 140 (2001) 418 [hep-ph/0012260] [INSPIRE].

[143] T. Hahn and C. Schappacher, The Implementation of the minimal supersymmetric standard model in FeynArts and FormCalc, Comput. Phys. Commun. 143 (2002) 54 [hep-ph/0105349] [INSPIRE].

[144] F. Staub, SARAH, arXiv:0806.0538 [INSPIRE].

[145] F. Staub, From Superpotential to Model Files for FeynArts and CalcHep/CompHEP, Comput. Phys. Commun. 181 (2010) 1077 [arXiv:0909.2863] [INSPIRE].

[146] F. Staub, Automatic Calculation of supersymmetric Renormalization Group Equations and Self Energies, Comput. Phys. Commun. 182 (2011) 808 [arXiv:1002.0840] [INSPIRE].

[147] T. Hahn and M. Pérez-Victoria, Automatized one loop calculations in four-dimensions and D-dimensions, Comput. Phys. Commun. 118 (1999) 153 [hep-ph/9807565] [INSPIRE].

[148] T. Hahn, A Mathematica interface for FormCalc-generated code, Comput. Phys. Commun. 178 (2008) 217 [hep-ph/0611273] [INSPIRE]. 
[149] F. del Aguila, A. Culatti, R. Muñoz Tapia and M. Pérez-Victoria, Techniques for one loop calculations in constrained differential renormalization, Nucl. Phys. B 537 (1999) 561 [hep-ph/9806451] [INSPIRE].

[150] P.Z. Skands et al., SUSY Les Houches accord: Interfacing SUSY spectrum calculators, decay packages and event generators, JHEP 07 (2004) 036 [hep-ph/0311123] [INSPIRE].

[151] B. Allanach et al., SUSY Les Houches Accord 2, Comput. Phys. Commun. 180 (2009) 8 [arXiv:0801.0045] [INSPIRE].

[152] F. Mahmoudi et al., Flavour Les Houches Accord: Interfacing Flavour related Codes, Comput. Phys. Commun. 183 (2012) 285 [arXiv: 1008.0762] [InSPIRE].

[153] U. Ellwanger, J.F. Gunion and C. Hugonie, NMHDECAY: A Fortran code for the Higgs masses, couplings and decay widths in the NMSSM, JHEP 02 (2005) 066 [hep-ph/0406215] [INSPIRE].

[154] U. Ellwanger and C. Hugonie, NMHDECAY 2.0: An Updated program for sparticle masses, Higgs masses, couplings and decay widths in the NMSSM, Comput. Phys. Commun. 175 (2006) 290 [hep-ph/0508022] [INSPIRE].

[155] U. Ellwanger and C. Hugonie, NMSPEC: A Fortran code for the sparticle and Higgs masses in the NMSSM with GUT scale boundary conditions, Comput. Phys. Commun. 177 (2007) 399 [hep-ph/0612134] [INSPIRE].

[156] http://www.th.u-psud.fr/NMHDECAY/nmssmtools.html.

[157] Particle Data Group collaboration, K. Nakamura et al., Review of particle physics, J. Phys. G 37 (2010) 075021 [inSPIRE].

[158] P. Bechtle, O. Brein, S. Heinemeyer, G. Weiglein and K.E. Williams, HiggsBounds: Confronting Arbitrary Higgs Sectors with Exclusion Bounds from LEP and the Tevatron, Comput. Phys. Commun. 181 (2010) 138 [arXiv:0811.4169] [INSPIRE].

[159] P. Bechtle, O. Brein, S. Heinemeyer, G. Weiglein and K.E. Williams, HiggsBounds 2.0.0: Confronting Neutral and Charged Higgs Sector Predictions with Exclusion Bounds from LEP and the Tevatron, Comput. Phys. Commun. 182 (2011) 2605 [arXiv:1102.1898] [InSPIRE].

[160] M. Spira, HIGLU: A program for the calculation of the total Higgs production cross-section at hadron colliders via gluon fusion including QCD corrections, hep-ph/9510347 [INSPIRE].

[161] http://people.web.psi.ch/spira/proglist.html.

[162] W. Beenakker, S. Dittmaier, M. Krämer, B. Plumper, M. Spira and P. M. Zerwas, Higgs radiation off top quarks at the Tevatron and the LHC, Phys. Rev. Lett. 87 (2001) 201805 [hep-ph/0107081] [INSPIRE].

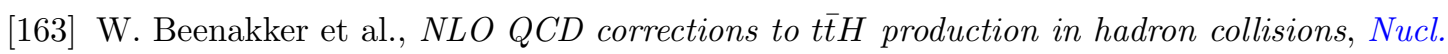
Phys. B 653 (2003) 151 [hep-ph/0211352] [INSPIRE].

[164] S. Dawson and L. Reina, QCD corrections to associated Higgs boson production, Phys. Rev. D 57 (1998) 5851 [hep-ph/9712400] [INSPIRE].

[165] L. Reina and S. Dawson, Next-to-leading order results for tth production at the Tevatron, Phys. Rev. Lett. 87 (2001) 201804 [hep-ph/0107101] [INSPIRE].

[166] S. Dawson, L. Orr, L. Reina and D. Wackeroth, Associated top quark Higgs boson production at the LHC, Phys. Rev. D 67 (2003) 071503 [hep-ph/0211438] [INSPIRE].

[167] LHC Higgs Cross Section Working Group collaboration, S. Dittmaier et al., Handbook of LHC Higgs Cross Sections: 1. Inclusive Observables, arXiv:1101.0593 [INSPIRE]. 
[168] https://twiki.cern.ch/twiki/bin/view/LHCPhysics/CrossSections.

[169] A. Djouadi, M. Spira and P. Zerwas, Production of Higgs bosons in proton colliders: QCD corrections, Phys. Lett. B 264 (1991) 440 [INSPIRE].

[170] A. Djouadi, M. Spira and P. Zerwas, QCD corrections to hadronic Higgs decays, Z. Phys. C 70 (1996) 427 [hep-ph/9511344] [INSPIRE].

[171] M. Spira, A. Djouadi, D. Graudenz and P. Zerwas, Higgs boson production at the LHC, Nucl. Phys. B 453 (1995) 17 [hep-ph/9504378] [inSPIRE].

[172] A. Djouadi, J. Kalinowski and M. Spira, HDECAY: A Program for Higgs boson decays in the standard model and its supersymmetric extension, Comput. Phys. Commun. 108 (1998) 56 [hep-ph/9704448] [INSPIRE].

[173] J. Butterworth et al., The Tools and Monte Carlo working group Summary Report, arXiv: 1003.1643 [INSPIRE].

[174] A. Djouadi, M. Muhlleitner and M. Spira, Decays of supersymmetric particles: The Program SUSY-HIT (SUspect-SdecaY-HDECAY-InTerface), Acta Phys. Polon. B 38 (2007) 635 [hep-ph/0609292] [INSPIRE].

[175] ATLAS collaboration, G. Aad et al., Search for squarks and gluinos using final states with jets and missing transverse momentum with the ATLAS detector in $\sqrt{s}=7 \mathrm{TeV}$ proton-proton collisions, Phys. Lett. B 701 (2011) 186 [arXiv:1102.5290] [INSPIRE].

[176] ATLAS collaboration, G. Aad et al., Search for supersymmetry in pp collisions at $\sqrt{s}=7$ $\mathrm{TeV}$ in final states with missing transverse momentum and b-jets, Phys. Lett. B 701 (2011) 398 [arXiv: 1103.4344] [INSPIRE].

[177] ATLAS collaboration, G. Aad et al., Search for squarks and gluinos using final states with jets and missing transverse momentum with the ATLAS detector in $\sqrt{s}=7 \mathrm{TeV}$ proton-proton collisions, Phys. Lett. B 710 (2012) 67 [arXiv:1109.6572] [inSPIRE].

[178] ATLAS collaboration, G. Aad et al., Search for supersymmetry in final states with jets, missing transverse momentum and one isolated lepton in $\sqrt{s}=7 \mathrm{TeV}$ pp collisions using 1 $\mathrm{fb}^{-1}$ of ATLAS data, Phys. Rev. D 85 (2012) 012006 [arXiv:1109.6606] [InSPIRE].

[179] ATLAS collaboration, G. Aad et al., Search for new phenomena in final states with large jet multiplicities and missing transverse momentum using $\sqrt{s}=7 \mathrm{TeV}$ pp collisions with the ATLAS detector, JHEP 11 (2011) 099 [arXiv:1110.2299] [INSPIRE].

[180] ATLAS collaboration, G. Aad et al., Search for scalar bottom pair production with the ATLAS detector in pp Collisions at $\sqrt{s}=7$ TeV, Phys. Rev. Lett. 108 (2012) 181802 [arXiv:1112.3832] [INSPIRE].

[181] CMS collaboration, S. Chatrchyan et al., Search for Supersymmetry at the LHC in Events with Jets and Missing Transverse Energy, Phys. Rev. Lett. 107 (2011) 221804 [arXiv: 1109.2352] [INSPIRE].

[182] The CMS collaboration, Search for supersymmetry in all-hadronic events with missing energy, PAS-SUS-11-004.

[183] The CMS collaboration, Search for supersymmetry in all-hadronic events with MT2, PAS-SUS-11-005.

[184] The CMS collaboration, Search for new physics with same-sign isolated dilepton events with jets and missing energy, PAS-SUS-11-010.

[185] The CMS collaboration, Interpretation of Searches for Supersymmetry, PAS-SUS-11-016. 
[186] G. Bélanger, F. Boudjema, A. Pukhov and A. Semenov, MicrOMEGAs: A Program for calculating the relic density in the MSSM, Comput. Phys. Commun. 149 (2002) 103 [hep-ph/0112278] [INSPIRE].

[187] G. Bélanger, F. Boudjema, A. Pukhov and A. Semenov, MicrOMEGAs: Version 1.3, Comput. Phys. Commun. 174 (2006) 577 [hep-ph/0405253] [INSPIRE].

[188] G. Bélanger, F. Boudjema, A. Pukhov and A. Semenov, Dark matter direct detection rate in a generic model with MicrOMEGAs 2.2, Comput. Phys. Commun. 180 (2009) 747 [arXiv: 0803.2360] [INSPIRE].

[189] G. Bélanger et al., Indirect search for dark matter with MicrOMEGAs2.4, Comput. Phys. Commun. 182 (2011) 842 [arXiv: 1004.1092] [INSPIRE].

[190] ATLAS collaboration, G. Aad et al., Search for stable hadronising squarks and gluinos with the ATLAS experiment at the LHC, Phys. Lett. B 701 (2011) 1 [arXiv:1103.1984] [INSPIRE].

[191] ATLAS collaboration, G. Aad et al., Search for supersymmetry in pp collisions at $\sqrt{s}=7$ $\mathrm{TeV}$ in final states with missing transverse momentum and b-jets with the ATLAS detector, Phys. Rev. D 85 (2012) 112006 [arXiv:1203.6193] [InSPIRE].

[192] ATLAS collaboration, G. Aad et al., Search for scalar top quark pair production in natural gauge mediated supersymmetry models with the ATLAS detector in pp collisions at $\sqrt{s}=7$ TeV, Phys. Lett. B 715 (2012) 44 [arXiv: 1204.6736] [INSPIRE].

[193] CMS collaboration, S. Chatrchyan et al., Search for new physics in events with same-sign dileptons and b-tagged jets in pp collisions at $\sqrt{s}=7 \mathrm{TeV}$, JHEP 08 (2012) 110 [arXiv: 1205.3933] [INSPIRE].

[194] The CMS collaboration, Search for supersymmetry in events with a single lepton and jets using templates, PAS-SUS-11-027.

[195] The CMS collaboration, Search for Supersymmetry in Final States with a Single Lepton, $B$-jets and Missing Transverse Energy in Proton-Proton Collisions at $\sqrt{s}=7 \mathrm{TeV}$, PAS-SUS-11-028.

[196] F. Gianotti (ATLAS collaboration) and J. Incandela (CMS collaboration), Latest update in the Search for the Higgs Boson, talks given at the conference Update on the search for the Higgs boson at CERN, CERN, Geneva, 4 July 2012 [http://indico.cern.ch/conferenceDisplay.py?confId=197461].

[197] ATLAS collaboration, Observation of an Excess of Events in the Search for the Standard Model Higgs boson with the ATLAS detector at the LHC, ATLAS-CONF-2012-093.

[198] The CMS collaboration, Observation of a new boson with a mass near $125 \mathrm{GeV}$, PAS-HIG-12-020. 